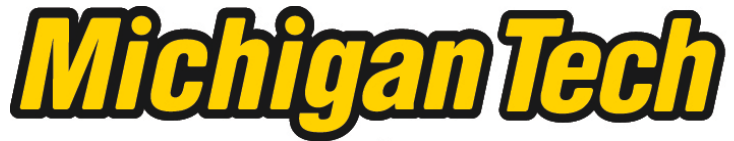 \\ Michigan Technological University Create the Future Digital Commons @ Michigan Tech
}

Dissertations, Master's Theses and Master's Reports - Open

Dissertations, Master's Theses and Master's

Reports

2014

\section{A Study of Passenger Car Acoustic Cavity Boom Simulation Methods}

Shreerang Dhabe

Michigan Technological University

Follow this and additional works at: https://digitalcommons.mtu.edu/etds

Part of the Mechanical Engineering Commons

Copyright 2014 Shreerang Dhabe

\section{Recommended Citation}

Dhabe, Shreerang, "A Study of Passenger Car Acoustic Cavity Boom Simulation Methods", Master's Thesis, Michigan Technological University, 2014.

https://doi.org/10.37099/mtu.dc.etds/756

Follow this and additional works at: https://digitalcommons.mtu.edu/etds

Part of the Mechanical Engineering Commons 


\title{
A STUDY OF PASSENGER CAR ACOUSTIC CAVITY BOOM SIMULATION METHODS
}

\author{
By \\ Shreerang Dhabe
}

\begin{abstract}
A THESIS
Submitted in partial fulfillment of the requirements for the degree of MASTER OF SCIENCE

In Mechanical Engineering
\end{abstract}

MICHIGAN TECHNOLOGICAL UNIVERSITY

2014

(C) 2014 Shreerang Dhabe 
This thesis has been approved in partial fulfillment of the requirements for the Degree of MASTER OF SCIENCE in Mechanical Engineering

Department of Mechanical Engineering-Engineering Mechanics

Thesis Co-Advisor: $\quad$ Dr. Gregory M. Odegard
Thesis Co-Advisor: $\quad$ Prof. Charles D. Van Karsen

Committee Member: Dr. James DeClerck

Committee Member: Prof. John L. Irwin

Department Chair: $\quad$ Dr. William W. Predebon 


\section{Table of Contents}

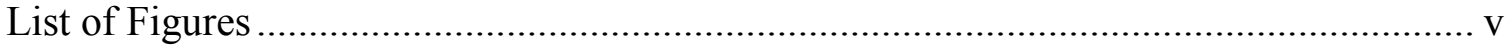

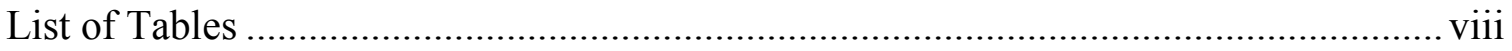

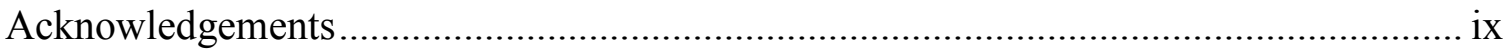

Abstract t.1.1.

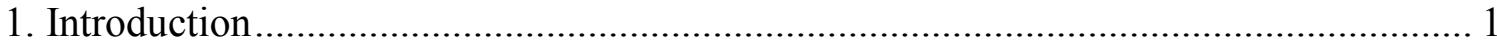

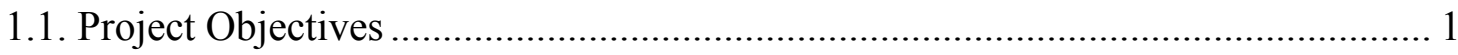

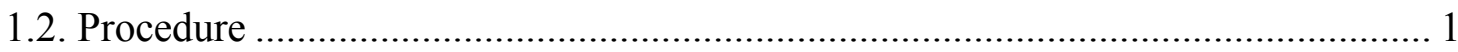

1.3. MTU computing and test facilities ................................................................. 2

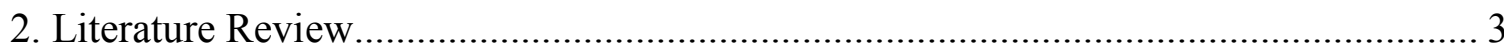

3. Finite Element Analysis of Chevrolet Cruze using Abaqus 6.12 ............................... 7

3.1. Chevrolet Cruze LTZ Structural Mesh .......................................................... 7

a. Eigen solution of structural mesh \& GM Optistruct result comparison................... 7

3.2. Chevrolet Cruze LTZ Acoustic Mesh........................................................... 13

3.2.1. Eigen solution of Acoustic Mesh \#1 \& GM Optistruct result comparison..... 15

3.2.2. Eigen solution of Acoustic Mesh \#2 \& GM Optistruct result comparison..... 17

3.3. Chevrolet Cruze LTZ Coupled Solution Analysis................................................. 18

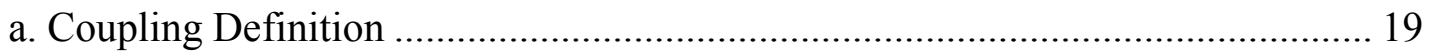

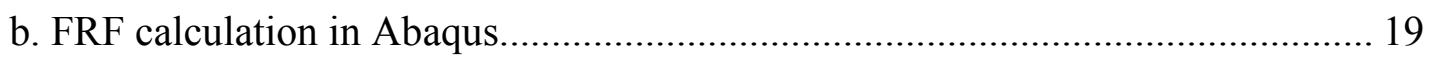

3.3.1. Coupled Solution \#1 Analysis .................................................................... 21

a. Eigen Solution of Cruze Coupled Solution \#1 …………..................................... 21

b. FRF calculation for Coupled Solution\#1 ……………...................................... 22

3.3.2. Cruze Coupled Solution \#2 ............................................................... 26

a. Eigen Solution of Cruze Coupled Solution \#2 …………................................. 26

b. FRF Calculation for Cruze Acoustic Mesh \#2 ................................................. 28

3.4. Comparison of Coupled Acoustic Solution \#1 and Coupled Solution \#2 ……..... 32

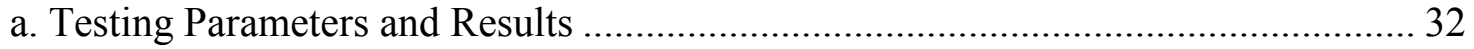

b. Comparison between coupled solutions................................................................ 32

4: Finite Element Analysis of Simple Car Models (SCM) ……………………………... 34

4.1. Simple Car Model \#1 (SCM\#1) ..................................................................... 34

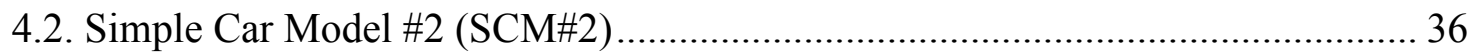

4.2.1. Simple Car Model \#2A (SCM \#2A) ……………...................................... 36 


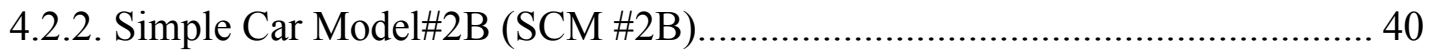

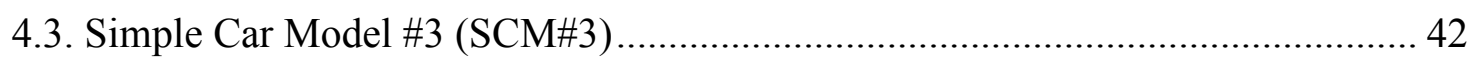

4.3.1. Simple Car Model \#3A (SCM\#3A) ............................................................ 42

4.3.2. Simple Car Model \#3B (SCM\#3B)............................................................. 44

4.3.3. Effect of door cavities on acoustic mode shapes ............................................ 46

4.3.4. Effect of door cavities on acoustic sensitivity at DRE .................................. 48

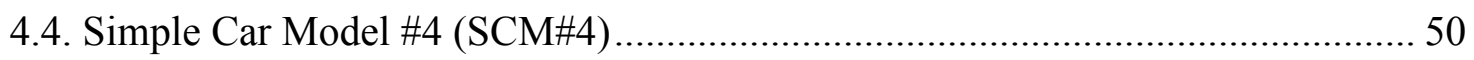

4.4.1. Simple Car Model \#4A (SCM \#4A) .......................................................... 50

4.4.2. Effect of addition of rear seats and rear package shelf speaker openings on

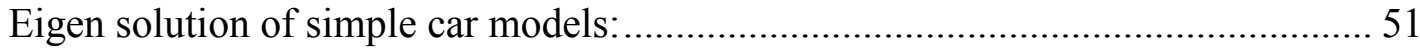

4.4.3. Simple Car Model \#4B (SCM \#4B) ............................................................. 52

4.4.4. Effect of addition of speaker openings on acoustic sensitivity at DRE.......... 53

4.4.5. Simple Car Model \#4C (SCM \#4C) ............................................................ 55

4.4.6. Simple Car Model \#4D (SCM \#4D) …………......................................... 56

4.4.7. Effect of different rear package shelf speaker opening diameter on the

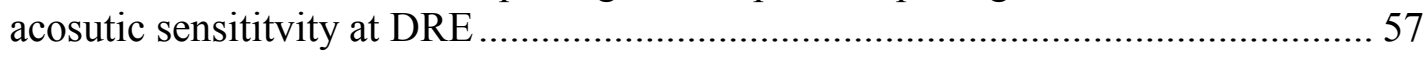

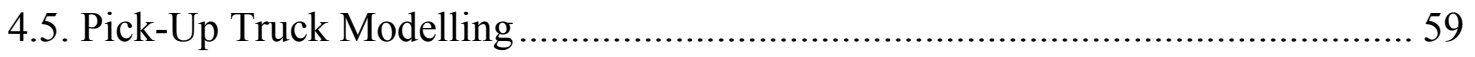

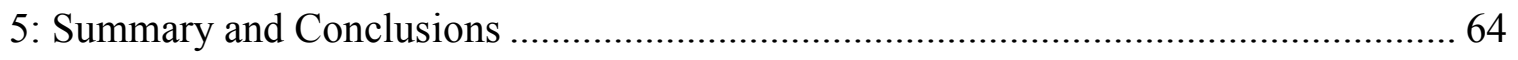

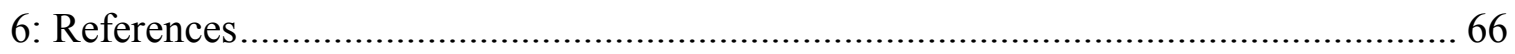




\section{List of Figures}

Figure 1: Schematic of Vibro-Acoustic Energy Flow [3] ........................................... 3

Figure 2: Chevrolet Cruze LTZ Structural Mesh......................................................... 8

Figure 3: Natural frequency comparison of Abaqus and Optistruct result ......................... 9

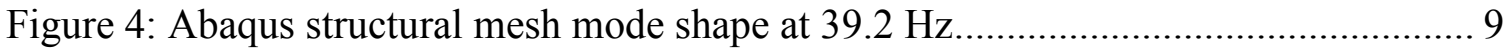

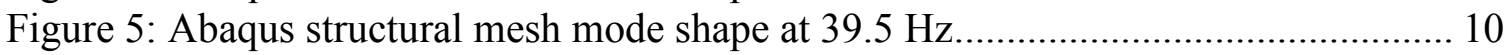

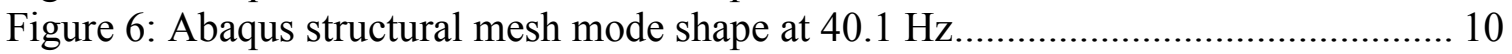

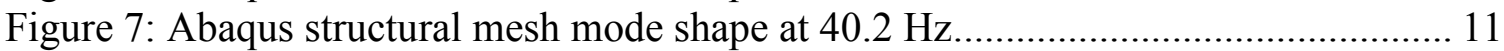

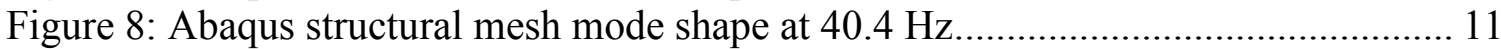

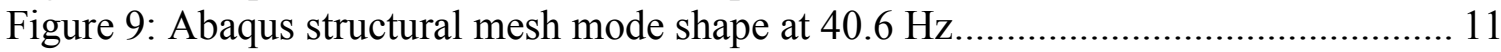

Figure 10: MAC comparison between GM Optistruct and Abaqus result........................ 12

Figure 11: Isometric view of MAC comparison between GM Optistruct and Abaqus

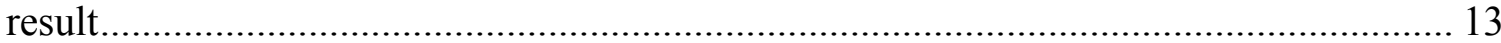

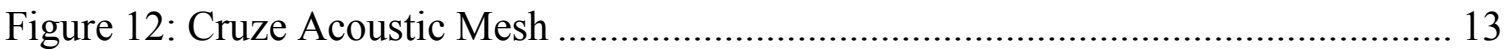

Figure 13: Component view of Cruze Acoustic Mesh showing rear seats ....................... 14

Figure 14: Top view of Acoustic Mesh \#1(left) and Acoustic Mesh \#2(right) showing

differences between two meshes.............................................................................. 15

Figure 15: Natural frequencies comparison between Abaqus and GM Optistruct result for Cruze Acoustic Mesh \#1 .................................................................................... 16

Figure 16: Mode Shape at $39 \mathrm{~Hz}$ for Acoustic Mesh \#1 ............................................ 16

Figure 17: Mode Shapes at $65 \mathrm{~Hz}$ for Acoustic Mesh \#1 ............................................. 17

Figure 18: Natural frequencies comparison between Abaqus and GM Optistruct result of

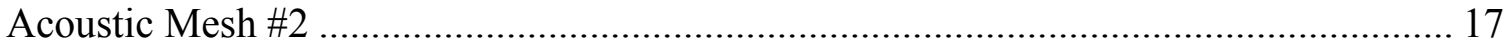

Figure 19: Mode Shape at $32 \mathrm{~Hz}$ of Acoustic Mesh \#2 ………….............................. 18

Figure 20: Mode Shape at $64.25 \mathrm{~Hz}$ of Acoustic Mesh \#2 ........................................... 18

Figure 21: Nodes missing intersection represented by red between the coupling surfaces

Figure 22: Structural input points on Cruze model for FRF generation .......................... 20

Figure 23: Structural output points on Cruze model for FRF generation ......................... 20

Figure 24: Natural frequency comparison between Structure Only and Coupled

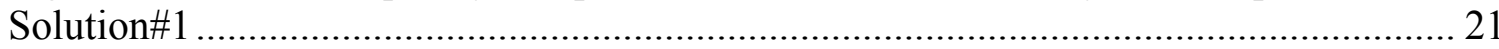

Figure 25: Coupled Mode Shapes at $39.7 \mathrm{~Hz}$ for Coupled Solution \#1 ……….............. 22

Figure 26: Coupled Mode Shapes at 41.1 Hz for GM Coupled Solution \#1 ................... 22

Figure 27: Drive point FRF for Coupled Solution \#1 ………...................................... 23

Figure 28: Comparison of acoustic sensitivity at DRE for Coupled Solution \#1 ............. 23

Figure 29: FRF showing acoustic sensitivity at Door Cavities due to Rear Input............ 24

Figure 30: FRF showing acoustic sensitivity at different points due to Front Input ........ 24

Figure 31: Drive point comparison between Structure Only Solution and Coupled

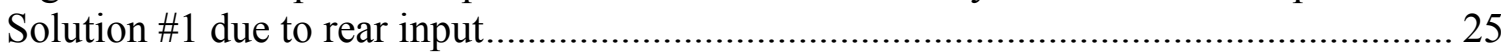

Figure 32: Comparison of Structure Only Solution and Coupled Solution \#1 for output at

Header Middle due to rear input ..................................................................................... 25

Figure 33: Modal Assurance Criteria between Coupled Structural Solution \#1 and

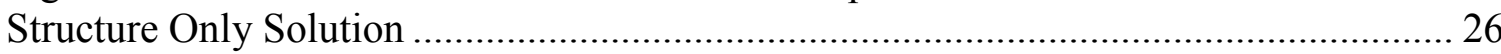


Figure 34: Natural frequencies comparison between Structural Only Sol and Coupled

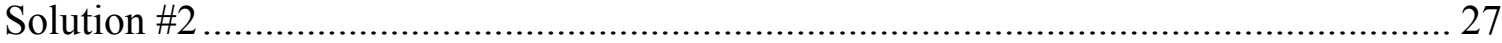

Figure 35: Coupled Mode Shapes at $38 \mathrm{~Hz}$............................................................. 27

Figure 36: Coupled Mode Shapes at $48.2 \mathrm{~Hz}$............................................................ 28

Figure 37: Drive Point FRF for Coupled Solution \#2 at front and rear input................... 28

Figure 38: Acoustic sensitivity at DRE due to front and rear input................................. 29

Figure 39: Acoustic sensitivities at door cavities due to front input................................. 29

Figure 40: Acoustic Sensitivity at different points due to rear input............................... 30

Figure 41: Drive point comparison between Structure Only Solution and Coupled

Solution \#2 due to rear input..................................................................................... 30

Figure 42: Comparison of Structure Only Sol and Coupled Solution \#2 for output at

Header Middle due to front input.................................................................................. 31

Figure 43: MAC Calculation between Coupled Solution\#2 and Structure Only Solution 31

Figure 44: MAC Plot between Coupled Solution\#1 and GM Coupled Solution\#2.......... 32

Figure 45: Comparison between Coupled Sol \#1 and Coupled Sol \#2 for acoustic

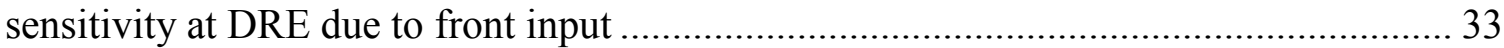

Figure 46: Geometrical Properties in meters (m) of structural mesh of SCM \#1 ............ 34

Figure 47: Structural Mesh of Simple Car Model\#1 ……………………………..... 35

Figure 48: Geometrical Properties in meters (m) of structural mesh of SCM \#2A.......... 36

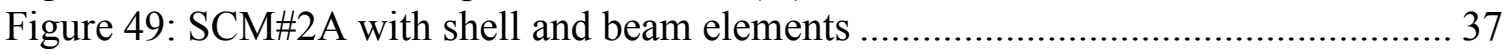

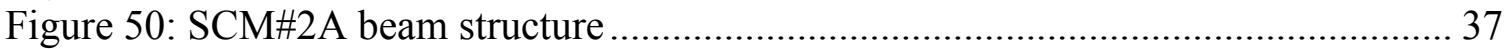

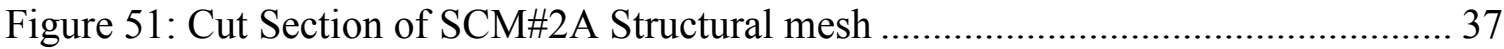

Figure 52: SCM \#2A Acoustic Mesh....................................................................... 38

Figure 53: MAC comparison between SCM \#2A pure acoustic and SCM \#2A coupled

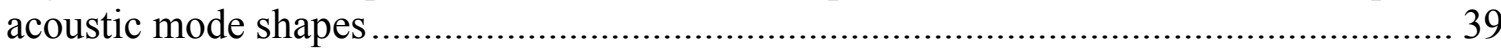

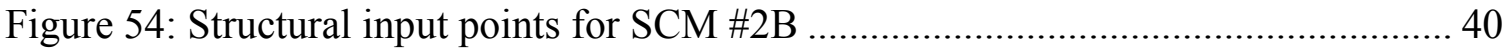

Figure 55: SCM \#2B Acoustic Mesh........................................................................ 41

Figure 56: MAC comparison between SCM \#2B pure acoustic and SCM\#2B coupled

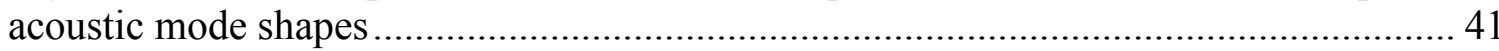

Figure 57: SCM \#3A Acoustic Mesh with one access for each door .............................. 42

Figure 58: Connections between door cavities and main acoustic cavity for SCM \#3A . 43 Figure 59: MAC comparison between SCM \#3A pure acoustic modes and SCM

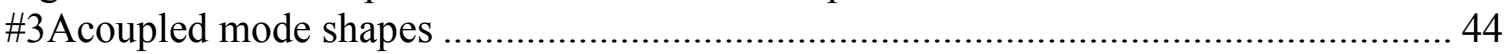

Figure 60: Simple Car Model \#3B acoustic mesh with two access for each door cavities

Figure 61: MAC plot between SCM \#3B pure acoustic modes and SCM \#3B coupled

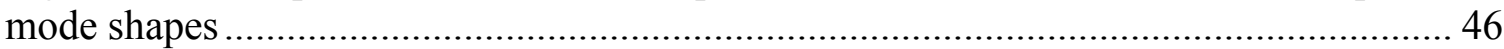

Figure 62: Mode shape comparison between SCM \#2A and SCM \#3A …..................... 47

Figure 63: Mode shape Comparison between SCM \#2A and SCM \#3B ........................ 48

Figure 64: Structural Input Points for SCM \#2 ......................................................... 48

Figure 65: Comparison of acoustic sensitivity at DRE due to front input for SCM \#2A,

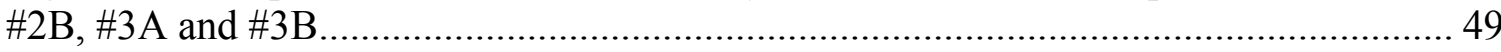

Figure 66: Comparison of acoustic sensitivity at DRE due to rear input for SCM \#2A,

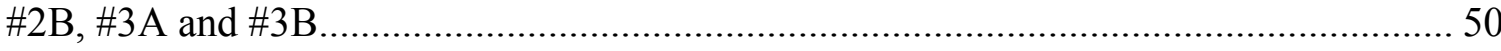


Figure 67: SCM \#4A Acoustic Mesh............................................................................. 51

Figure 68: Mode shape comparison between SCM \#2A and SCM \#4A ........................ 52

Figure 69: SCM \#4B Acoustic Mesh....................................................................... 53

Figure 70: Acoustic sensitivity at DRE due to front input between SCM \#4A and SCM

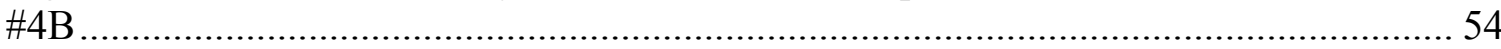

Figure 71: Acoustic sensitivity at DRE due to rear input between SCM \#4A and SCM

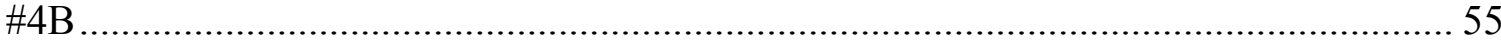

Figure 72: Different views of SCM \#4C Acoustic Mesh ............................................ 55

Figure 73: Different Views of SCM \#4D Acoustic Mesh ............................................ 56

Figure 74: Comparison of acoustic sensitivity between SCM \#4B, \#4C and \#4D due to

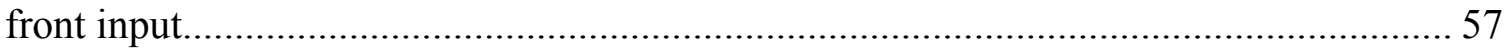

Figure 75: Comparison of acoustic sensitivity between SCM \#4B, \#4C and \#4D due to

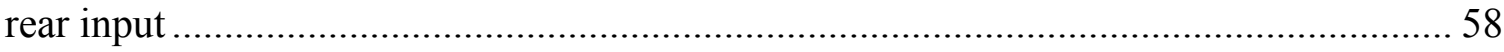

Figure 76: Simplified figure showing role of speaker openings for SCM \#2B ............... 58

Figure 77: Dimensions in meters (m) of Simple Pick-Up Truck Model ........................ 59

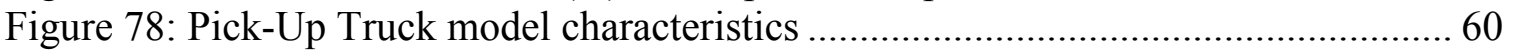

Figure 79: Pick-Up Truck model showing the structural input and acoustic output

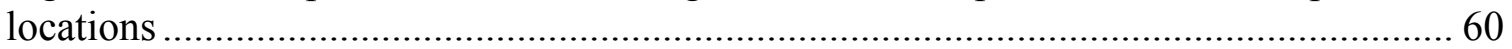

Figure 80: Tie Constraints for coupling on the pick-cup truck model........................... 61

Figure 81: Acoustic sensitivity at DRE due to force on truck bed ................................ 62

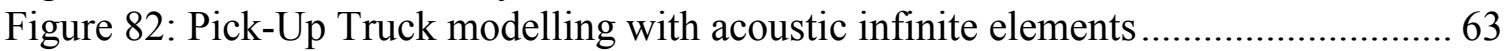

Figure 83: Acoustic Sensitivity at DRE for different air gap thickness values ............... 63 


\section{List of Tables}

Table 1: Summary of commercial implementation of vibro-acoustic coupling solution ... 6

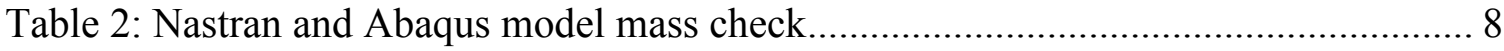

Table 3: Structural Mesh attributes of Simple Car Model \#1 ......................................... 35

Table 4: Acoustic Mesh attributes of Simple Car Model \#1 ........................................ 35

Table 5: Structural Mesh attributes of Simple Car Model\#2 .......................................... 37

Table 6: Acoustic Mesh attributes of Simple Car Model\#2A......................................... 39

Table 7: Acoustic Mesh attributes of Simple Car Model\#2B ....................................... 40

Table 8: SCM \#3A Acoustic Mesh Properties........................................................... 43

Table 9: SCM \#3A Acoustic Mesh attributes ............................................................. 45

Table 10: Comparison of acoustic natural frequencies for SCM \#2A, SCM \#3A and SCM

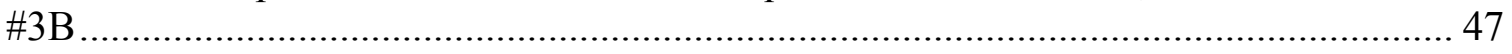

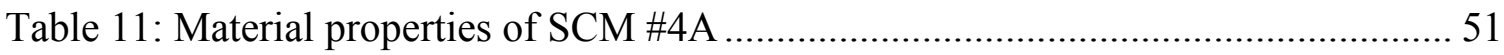

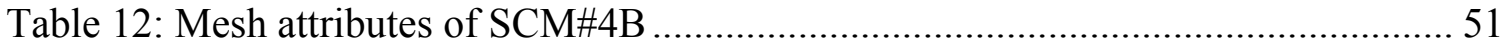

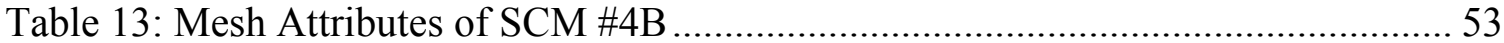

Table 14: Natural frequency comparison for SCM \#2A and SCM \#4B ......................... 52

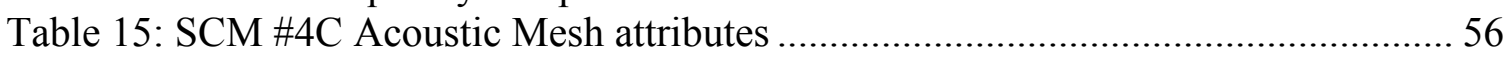

Table 16: SCM \#4D Acoustic Mesh attributes ............................................................. 57 


\section{Acknowledgements}

I would like to thank my advisers at Michigan Tech, for all the help, advice and guidance throughout the project. Thank you Dr. Gregory Odegard for giving me opportunity to work on this project. Thank you Prof. Charles Van Karsen for all the help with NVH concepts and fun in the project. Thank you Dr. James DeClerck for your help with presentations and your attention to detail. I have the utmost respect for you and you have made me the person that I am today. Also, I would like to thank Prof. John L. Irwin for agreeing to be on my committee.

I would like to thank project sponsors General Motors and Mr. Kurt Schneider, Mr. Robert Scheidt, Mr. Christopher Stirlen, Mr. Joseph Schudt from General Motors for all the help and patience throughout this project. The project wouldn't be possible without your inputs and direction. Also, Mr. Charlie Chin from Abaqus for his help with the software.

I would like to thank my colleagues at Michigan Tech. Mr. Mayuresh Pathak for always for his guidance with NVH concepts and for collecting the experimental data. Thank you Mr. Gaurav Agnihotri for all the help with post-processing of data.

I would also like to thank all my friends here at Michigan Tech. Lastly, I would like to thank my family back home for their unyielding support. I would like to specially thank my mom for her words of encouragement, help and support. Mom, you are the only constant in this ever changing world. It simply wouldn't have been possible without you.

No journey is complete without the characters involved. Once again, thank you all. 


\begin{abstract}
The project objective is the study of acoustic boom phenomenon at $40 \mathrm{~Hz}$ in Chevrolet Cruze LTZ and recommend modeling techniques to improve accuracy of model prediction. Acoustic boom is caused by solid-fluid interaction between car structure and air inside the car cavity.

In this report, finite element analysis software Abaqus 6.12-3 is used to simulate the acoustic-structural coupling. The structural and acoustic mesh for the Chevrolet Cruze LTZ are fully coupled and solved using Abaqus 'strongly coupled' solution. In order to understand the physics of acoustic boom in a car simple car box models were designed. Simple box car models were useful to find the effect of door cavities, rear seats and speaker openings on door cavities and rear package shelf on the acoustic boom phenomenon. Also, pick-up truck was modeled and acoustic response at driver's right ear was evaluated for a structural input on truck bed.

Finally, Abaqus results for the Chevrolet Cruze LTZ were compared to the Optistruct test results and specific method of modelling acoustic mesh was recommended. Also, analysis of simple car models led to significant insight into the physics of vibro-acoustic coupling and the effect of specific components on the acoustic response at the driver right ear.
\end{abstract}




\section{Introduction}

In the highly competitive automotive industry, there is an ever increasing need to reduce interior noise in passenger vehicle cabin which is important quality issue for a passenger vehicle [1]. The increase in interior sound pressure causes an undesirable booming noise at lower frequencies of 0 to $200 \mathrm{~Hz}$ and is induced due to the road or powertrain input. An acoustic boom is a complex phenomenon caused due to the interaction of the car structure and the air inside. Historically, it has been difficult to predict this phenomenon as multitude of design features interact to produce this noise. In the design of cars, finite element software's are used to determine the level of vibration and the acoustic levels inside a car.

In this research, finite element software Abaqus 6.12-3 is used to simulate the acousticstructural coupling in order to study the physics of acoustic boom in a Chevrolet Cruze LTZ over a frequency range of 0 to $300 \mathrm{~Hz}$. During testing on the Cruze, a booming noise was detected at $40 \mathrm{~Hz}$ frequency. Acoustic and structural mesh finite element models and their respective solutions in Optistruct (Altair) solver were provided by General Motors. The structural model of Chevrolet Cruze consisted of a fine mesh and 1.5 million nodes and an acoustic mesh consisted of relatively coarse mesh with 31 thousand elements. In order to validate the finite element results, experimental data was collected on the bodyin-white (BIW) model of Chevrolet Cruze and was correlated with the computational results. Mode shape comparison was performed using acoustic and vibrations simulation software, LMS Virtual.Lab.

Many small components interact to produce the acoustic boom inside a car cavity. Out of these components, the door cavities and the trunk have a relatively large hollow volume and so can be significant contributors to the acoustic boom phenomenon. So, in order to study the effect of these components, simple box car models were created using Altair Hypermesh and then were processed using finite element software Abaqus 6.12. All the post-processing was carried out in the Altair Hyperview software as it enabled viewing and comparison of Abaqus output file (.odb) and Nastran output file (.op2). Also, a simple box model of pick-up truck was designed to evaluate the capability of Abaqus to predict the acoustic sensitivity across the air gap between truck bed and truck cabin for a force applied on the truck bed. For all the analysis, the structural displacements are measured in meters (m) and acoustic sensitivities are measured in Pascal's (Pa).

\subsection{Project Objectives}

a) Model the physics of acoustic boom phenomenon in a passenger vehicle using Abaqus 6.12

b) Apply these methods to the Chevrolet Cruze LTZ body-in-white (BIW) model

\subsection{Procedure}

a) Simulate the acoustic-structural coupling of the Chevrolet Cruze car models using Abaqus 6.12

b) Baseline comparison of Abaqus 6.12 results with Optistruct results 
c) Evaluation of frequency response functions(FRF's) on various output points of the structural and acoustic meshes with structural input at the front and rear of the vehicle

d) Validation of finite element results of Chevrolet Cruze acoustic-structural model using experimental data

e) Construct simple box car models to study the effect of door cavities, rear seats and speaker openings in door cavities and the rear package shelf on acousticstructural coupling phenomenon

\subsection{MTU computing and test facilities}

The project was carried over a period of one year from September 2012 to September 2013 at Michigan Technological University. An overview of the various Michigan Tech facilities used is given below.

Abaqus 6.12: Abaqus is a suite of finite element software from Dassault Systems which offers design, solving and post processing capabilities. Abaqus 6.12-3 with a commercial license which offers simulation capabilities for a large number of nodes was used for this research. It offers unique capability in modelling the acoustic-structural coupling phenomenon and facilitates separate viewing of coupled-acoustic and coupled-structural mode shapes.

ATHENA computer cluster: In order to perform simulation of the huge six million degree of freedom model of Chevrolet Cruze, a computer cluster called 'ATHENA' was used. ATHENA is a computer cluster in Mechanical Engineering Department of Michigan Tech. It has 28 computer nodes, each equipped with 12 CPU cores and 24 GB RAM.

Hemi-Anechoic Chamber - Advanced Technology Development Centre: Testing was performed on the full Chevrolet Cruze LTZ (X70086EX, VIN: 1g1PK5s98B70086EX) at a hemi-anechoic chamber at Advanced Technology Development Complex (ATDC) at Michigan Tech. It has an area of 1350 sq. $\mathrm{ft}$. and has 2481 tetrahedral cones with a base area of 1sq. $\mathrm{ft}$ and a height of $3 \mathrm{ft}$ to absorb and deflect the sound. The engine, powertrain and the wheels were removed from the car, as they do not contribute to the acoustic boom phenomenon.

Data Acquisition System and Transducers: Data acquisition was performed using the LMS Test.lab 12A software and LMS SCADAS data acquisition system. Full structural modal test, full acoustic modal test with structural input, acoustic modal test with acoustic input and full acoustic modal testing to assess the effect of door cavities, rear seats and package shelf speakers were performed. PCB accelerometers and microphones from 'the modal shop' were used for data acquisition.

The report consists of 5 chapters. Chapter 2 includes literature review, chapter 3 deals with finite element analysis of the full Chevrolet Cruze LTZ model, chapter 4 deals with the modelling and analysis of simple box car models, chapter 5 contains the overall summary and conclusions. 


\section{Literature Review}

Internal noise that a driver experiences is caused due to various sources. Phil Shorter [2] classifies the various internal noise sources as air-borne, wind noise and structure-borne noise sources. The air-borne source causes an internal noise in the range of $315 \mathrm{~Hz}$ to 10 $\mathrm{kHz}$ with powertrain and road/chassis noise sources. The wind source causes internal noise in the range of $50 \mathrm{~Hz}$ to $10 \mathrm{kHz}$ and is caused due to aerodynamic noise sources. Whereas, the structure-borne noise is in the range of $0 \mathrm{~Hz}$ to $500 \mathrm{~Hz}$ and is caused due to the body inputs from powertrain and/or road or chassis. In this project, a structure-borne noise causes an acoustic boom at $40 \mathrm{~Hz}$ in a Chevrolet Cruze LTZ according to test results.

In order to reduce interior noise, the dynamic characteristic of Chevrolet Cruze LTZ must be studied and how the structure interacts with air inside. According to Van Karsen et al. [3] in the report submitted to General Motors, Vibro-Acoustic coupling as vibration energy transfer between entities with significantly different stress mechanisms and/or different densities. Generally, the energy transfer is between a metallic structure, which has nonuniform stress distribution and a fluid, which has uniform stress distribution and lower density than the metallic structure. A schematic of the energy flow is given in Figure 1[3]. The energy flow is in the direction of reduction in impedance to energy flow. For example, an audio speaker has a coil that provides structural input to a cone which moves air causing sound waves to propagate through the fluid medium. The mechanism of the speaker cone moving air is simply represented by a "feedback" block. Similarly, sound waves are converted back to mechanical motion in the ear. In these simple examples, the energy flow is usually one-way; from speaker to air then air to ear drum.

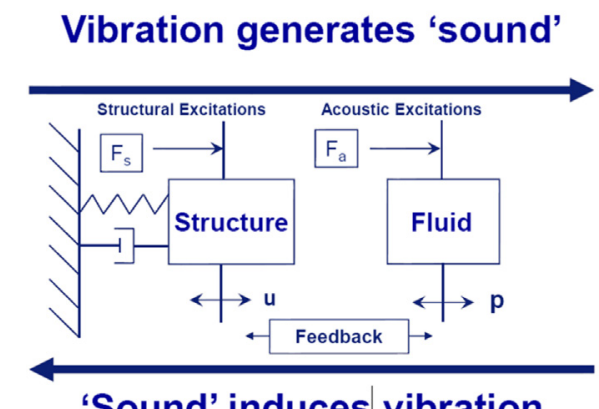

'Sound' induces vibration

Figure 1:Schematic of Vibro-Acoustic Energy Flow [3]

For automobiles, the structure and fluid (enclosed cabin air cavity) have independent dynamic characteristics, or modes, resulting in bands of high and low impedance across the frequency range of interest. All structural excitations are amplified and attenuated from source to vibro-acoustic interface through the structural modes. Vibration energy transferred to the air cavity is accepted or blocked depending on the cavity impedance at the interface which depends on the fluid density and cavity modes. The resultant effect is that some structural vibrations cause unwanted sound and some do not. 
Depending on the interface impedance and the fluid-structure interaction, the vibroacoustic coupled system may not have a simple mapping between the uncoupled and coupled systems. Natural frequency and mode shape changes depend on whether the coupling causes the fluid mass to move or be compressed. Moving fluid has a more significant mass effect, causing reduction in natural frequency and may or may not change the mode shape. If the structure motion compresses the fluid, like a balloon, natural frequency increases and the mode shape may or may not change.

Due to the difference in stress distribution and density, the approach to modeling vibroacoustic coupling is different than structure-structure or fluid-fluid coupling. Nefske et al. [4] in 1982 formulated the fully coupled problem by including boundary surface acceleration vector in the equation of motion of air and a boundary pressure vector in equations of motion of the structure. They stated that these two equations should be solved simultaneously. Yashiro et al. [5] gave a mathematical description of the fully coupled solution to an acoustic structural coupling problem as shown in equation (1).

$\left[\begin{array}{cc}M_{a a} & 0 \\ M_{c a} & M_{c c}\end{array}\right]\left\{\begin{array}{l}\ddot{X} \\ \ddot{P}\end{array}\right\}+\left[\begin{array}{cc}K_{a a} & K_{a c} \\ 0 & K_{c c}\end{array}\right]\left\{\begin{array}{l}X \\ P\end{array}\right\}=\left\{\begin{array}{l}F \\ 0\end{array}\right\}$

where,

$\left[M_{a a}\right]=$ body mass matrix

$\left[K_{a a}\right]=$ body stiffness index

$\left[M_{c c}\right]=$ matrix of interior sound field mass

$\left[K_{c c}\right]=$ matrix of interior sound field stiffness

$\left[M_{a c}\right]=$ coupled body and interior sound field mass matrix

$\left[K_{a c}\right]=$ coupled body and interior sound field stiffness matrix

$\{\mathrm{X}\}=$ body displacement vibration

$\{\mathrm{P}\}=$ sound pressure

$\{\mathrm{F}\}=$ exciting force applied to the body

These equations have a high solution cost as they tend to produce non-symmetrical matrices. Also, due to addition of structural and acoustic degrees of freedom the total number of degrees of freedom are considerably increased. Various commercially available vibro-acoustic solvers have embedded assumptions to reduce solution time of equation (1). FEA solvers use special algorithms to take advantage of sparse-symmetric matrices to reduce solution cost. A computationally efficient solution approach begins with modal solutions of individual subsystems followed by projection of the coupling terms in a way that establishes connections between the modes of the uncoupled subsystems. To reduce computation, only 20-50 percent of the subsystem modes are used. This solution approach yields very good accuracy in the frequency range of interest if the connections are not extraordinarily stiff (elastomeric mounts rather than welds with high bending moment connections) and maintain symmetry of the system matrices. 
$\mathrm{N}$ Lalor and $\mathrm{H}-\mathrm{H}$ Priebsch [6] in their literature review of interior noise over last 40 years conclude that currently finite element method is the most accurate and popular tool to predict interior noise for low frequencies. All the finite element software's solve very large number of equations to evaluate the displacements or acoustic pressures. Lalor, $\mathrm{N}$ et al. give an overview of methods to solve these equations. All solutions start with the equation of motion in the harmonic form as shown in equation (2).

$\left[-\omega^{2} \cdot M+j \omega \cdot C+K\right] \cdot\{X\}=\{F\}$

where,

$\mathrm{M}=$ mass matrix

$\mathrm{C}=$ viscous damping matrix

$\mathrm{K}=$ stiffness matric

$\mathrm{F}=$ force amplitude vector

Abaqus 6.12 uses direct method to solve equation (2) [7]. In direct method $\{\mathrm{F}\}$ is premultiplied by inverse of $\left[-\omega^{2} . M+j \omega . C+K\right]$ at each frequency of interest $(\omega)$ to obtain the value of $\{\mathrm{X}\}$. Abaqus 6.12 Documentation [7] discusses simulation of acoustic-structural coupling by tying the acoustic and structural surfaces together using single degree of freedom elements called tie constraints. So, the force or pressure applied on the structural side is transmitted on to the acoustic mesh and vice versa. The 'frequency' step in Abaqus is used to evaluate the Eigen solution. Two types of coupling methods, 'strongly coupled' and 'weakly coupled' methods are used for simulation of vibro-acoustic coupling. Optistruct is a finite element solver used by general motors to simulate acoustic structural coupling. Table 1 contains a summary of methods, assumptions, strengths and weaknesses for vibro-acoustic solvers from Altair (Optistruct) and Abaqus. The Abaqus 'strongly coupled' solution was used for all the subsequent analysis in this report as the 'strongly solution' results were in correlation with the test results. 
Table 1: Summary of commercial implementation of vibro-acoustic coupling solution

\begin{tabular}{|c|c|}
\hline Method & Procedure \\
\hline $\begin{array}{l}\text { Optistruct } \\
\text { "Weakly-Coupled" } \\
\text { Solution }\end{array}$ & $\begin{array}{l}\text { - Coordinate transformation moves coupling terms to the } \\
\text { system damping matrix } \\
\text { - Coupling then projected onto a truncated set of the } \\
\text { uncoupled normal modes } \\
\text { - Cannot visualize acoustic shapes for the coupled } \\
\text { system because they are not computed }\end{array}$ \\
\hline $\begin{array}{l}\text { Abaqus } \\
\text { "Weakly-Coupled" } \\
\text { Solution }\end{array}$ & $\begin{array}{l}\text { - Similar to Optistruct "Weakly Coupled" solution } \\
\text { - }\end{array}$ \\
\hline $\begin{array}{l}\text { Abaqus } \\
\text { "Strongly- } \\
\text { Coupled" Solution }\end{array}$ & $\begin{array}{l}\text { - Fully coupled modes are calculated at the outset } \\
\text { - Used when solid-fluid interaction is central to } \\
\text { vibrational behavior of solid and acoustic media } \\
\text { - Requires much more computation time }\end{array}$ \\
\hline
\end{tabular}

Avitable et al. [14] discusses various correlation techniques. Modal Assurance Criteria is used to validate software result using test results and comparison between two solvers. Ward Haylen [8], in his book describes Modal Assurance Criteria (MAC) as a degree of correlation between two vectors. MAC is formulated as follows:

$\operatorname{MAC}_{\mathrm{ij}}=\frac{\left[\left\{U_{i}\right\}^{T}\left\{e_{j}\right\}\right]^{2}}{\left[\left\{U_{i}\right\}^{T}\left\{U_{i}\right\}\right]\left[\left\{e_{j}\right\}^{T}\left\{e_{j}\right\}\right]}$

MAC values are between 0 to 1 and 0 to $100 \%$ with a high value of MAC showing high correlation whereas a low MAC value representing low correlation between the two vectors.

Thus, a finite element method approach is used, with the help of all the tools mentioned above in this report to simulate the acoustic-structural coupling and understand acoustic boom at low frequencies according to the procedure outlined previously. 


\section{Finite Element Analysis of Chevrolet Cruze using Abaqus 6.12}

According to the project objectives specified, finite element analysis of Chevrolet Cruze LTZ model is performed. One structural mesh and two acoustic meshes were provided by General Motors (GM) for analysis. Firstly, structural and acoustic meshes are solved individually and then the coupled acoustic-structural mesh is solved. The Abaqus solution for the structure and acoustic meshes is compared with the Optistruct (Altair) solution provided by General Motors. Finally, structural and acoustic frequency response is calculated on various points of car mesh with force applied at two structural inputs.

The general procedure in Abaqus 6.12 used to solve the structural, acoustic and coupled solution is described in three steps as follows:

Step 1: Model conversion from Nastran (.bdf) file to Abaqus (.inp) file. The Cruze mesh (.bdf) file was translated to Abaqus (.inp) file using the command "Abaqus fromNastran' in Abaqus command line. This command converts all the different elements in Optistruct/Nastran to equivalent Abaqus elements.

Step 2: Now, the converted (.inp) file is imported into Abaqus CAE. Using the 'Linear Perturbation' procedure, 'Frequency' step and 'Lanczos' solver in Abaqus, natural frequencies and mode shapes of structural mesh are extracted up to $60 \mathrm{~Hz}$. A baseline comparison between Abaqus and GM Optistruct result is performed.

Step 3: Finally, a 'Modal Steady State Dynamics' step is defined to calculate frequency response functions (FRF) with a frequency resolution of $0.1 \mathrm{~Hz}$. Two input points were defined on the structural mesh to mimic the full structural modal MIMO (multiple input and multiple output) test conditions on the Chevrolet Cruze LTZ and output is evaluated on many points on the structural and acoustic mesh. Also, visual comparison with the test results is performed.

\subsection{Chevrolet Cruze LTZ Structural Mesh}

\section{a. Eigen solution of structural mesh \& GM Optistruct result comparison}

A Chevrolet Cruze body-in-white (BIW) structural mesh modeled in Altair Hypermesh and stored as a bulk data file (.bdf) file was provided. Figure 2 shows the isometric view of the structural mesh. It is a huge finite element model consisting of 1.5 million number of nodes, 1.6 million number of elements, 1192 components and 1010 different material properties. 


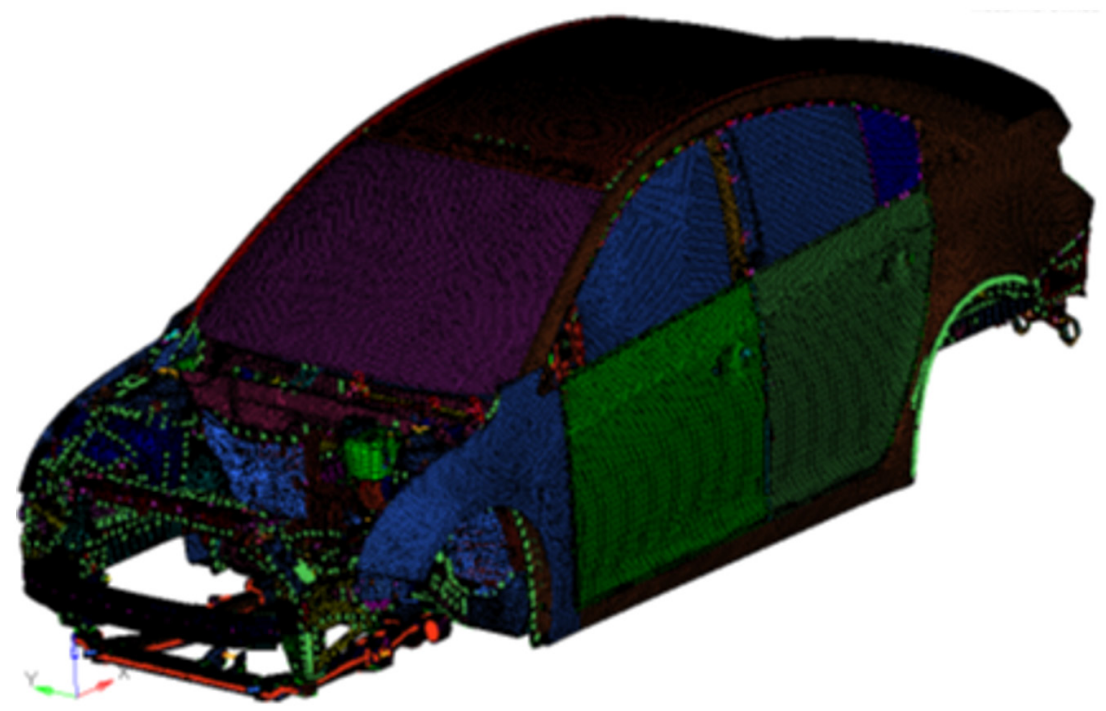

Figure 2: Chevrolet Cruze LTZ Structural Mesh

As shown in figure 2, engine and powertrain components are removed from the model. Most of the materials include different types of steel and glass for the car panels and windows respectively. Structural damping was provided in material properties.

Table 2: Nastran and Abaqus model mass check

\begin{tabular}{|c|c|c|}
\hline & Nastran & Abaqus \\
\hline Total Mass & $0.921932 \times 10^{3} \mathrm{Kg}$ & $0.9219546 \times 10^{3} \mathrm{Kg}$ \\
\hline Center of Mass & $(3107,-19.11,540.2)$ & $(3106.531,-19.109,540.21)$ \\
\hline
\end{tabular}

Table 2 shows the mass comparison between the original Nastran (.bdf) file and translated Abaqus (.inp) file. As shown, the model in both the software's has the same mass and center of mass and thus, the Nastran to Abaqus file conversion was successful with no loss of mass.

General Motors also provided the natural frequency and mode shapes obtained from solving the structural mesh in the Optistruct solver up to $60 \mathrm{~Hz}$. The Abaqus and Optistruct solution are compared and plotted as shown in figure 3. 


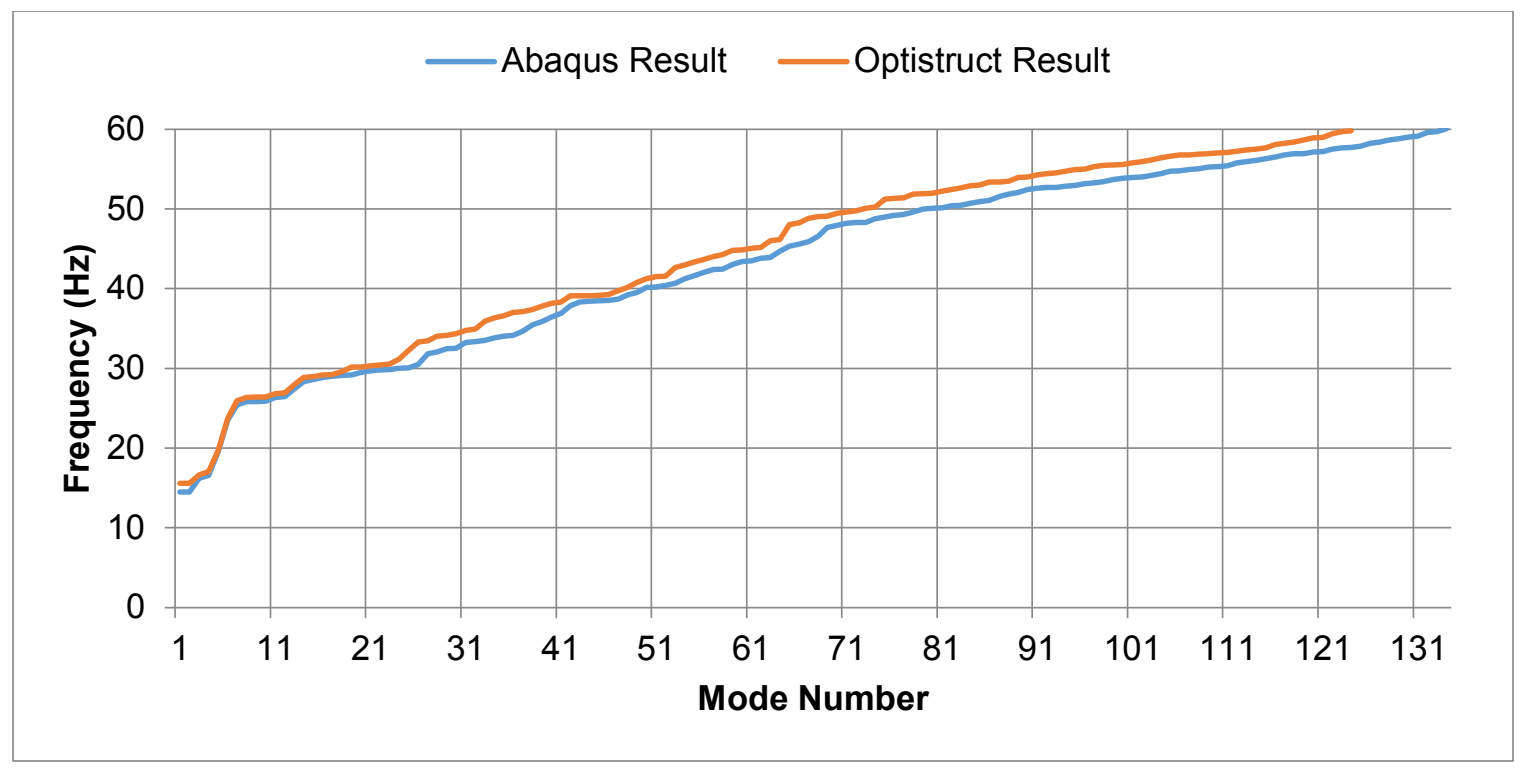

Figure 3: Natural frequency comparison of Abaqus and Optistruct result

Figure 3 shows the natural frequency comparison between Abaqus and Optistruct result. As shown, natural frequency is plotted in the range of $10 \mathrm{~Hz}$ to $60 \mathrm{~Hz}$. Optistruct results is found to be stiffer than the corresponding Abaqus result. Also, Abaqus estimates larger number of natural frequencies in the same frequency range as compared to Optistruct result. The mode shapes of the structural mesh were also evaluated using Abaqus and are shown below.
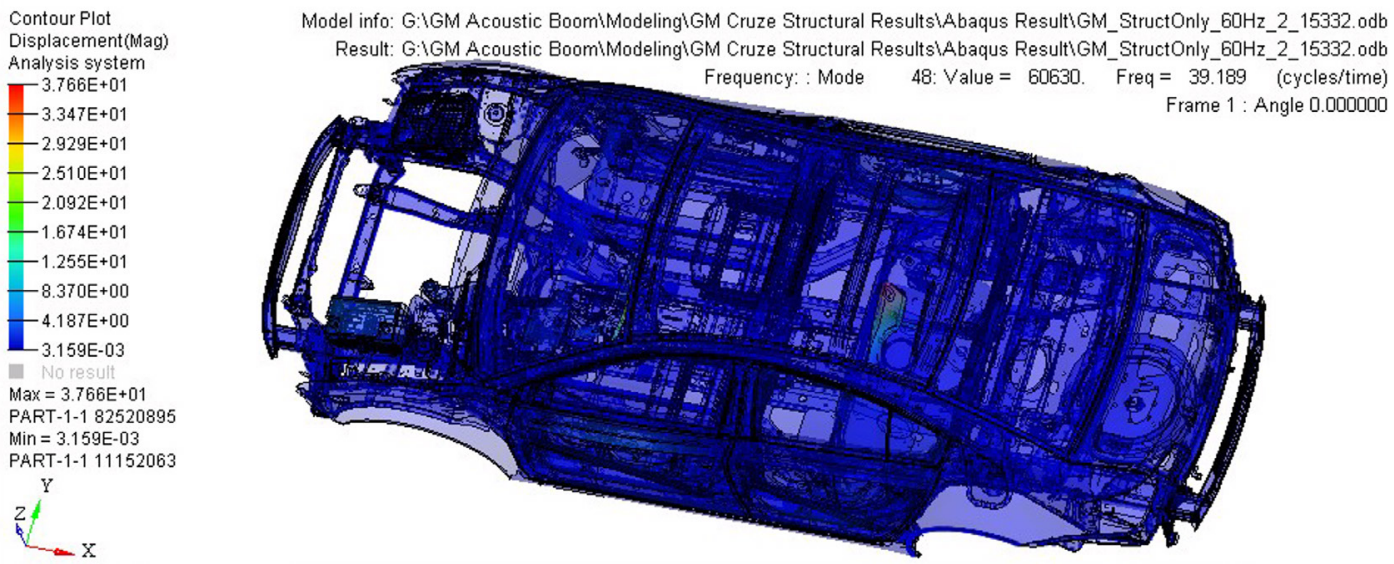

Figure 4: Abaqus structural mesh mode shape at $39.2 \mathrm{~Hz}$ 

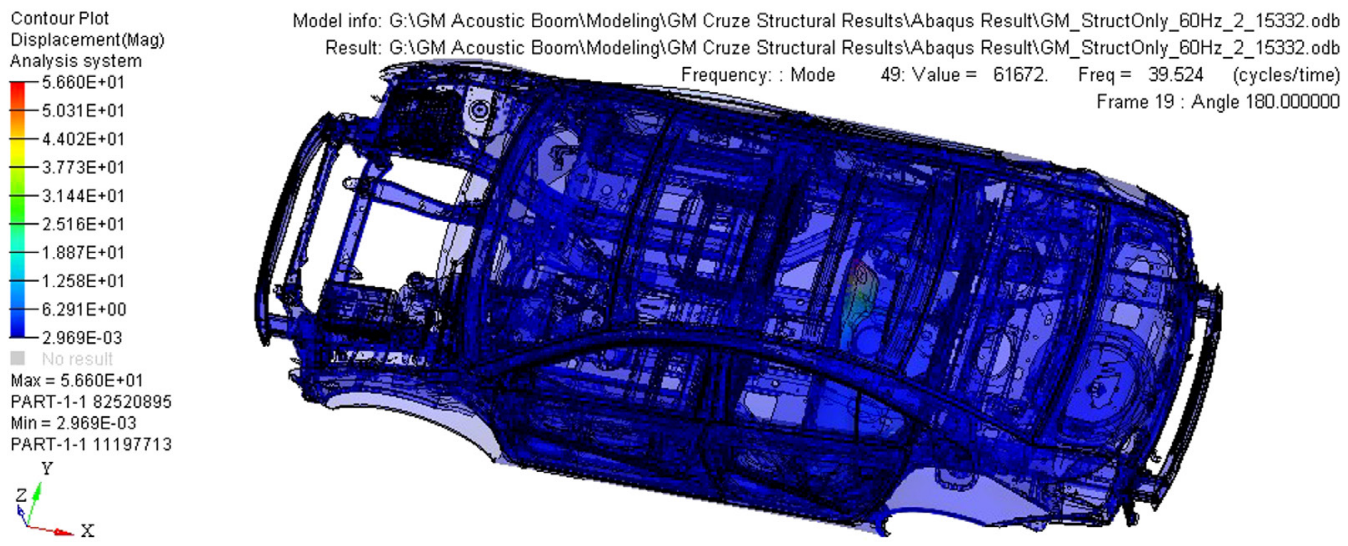

Figure 5: Abaqus structural mesh mode shape at $39.5 \mathrm{~Hz}$

Figure 4 and Figure 5 shows mode shapes around the boom frequency of $40 \mathrm{~Hz}$. As shown, the major component in both the mode shapes is the movement of the bottom plate on the floor of the car. Comparing it with optitsruct mode shape results, the movement of the bottom plate is not seen and it is suspected that during the conversion from Optistruct to Abaqus the connectors of the plate were not translated correctly.
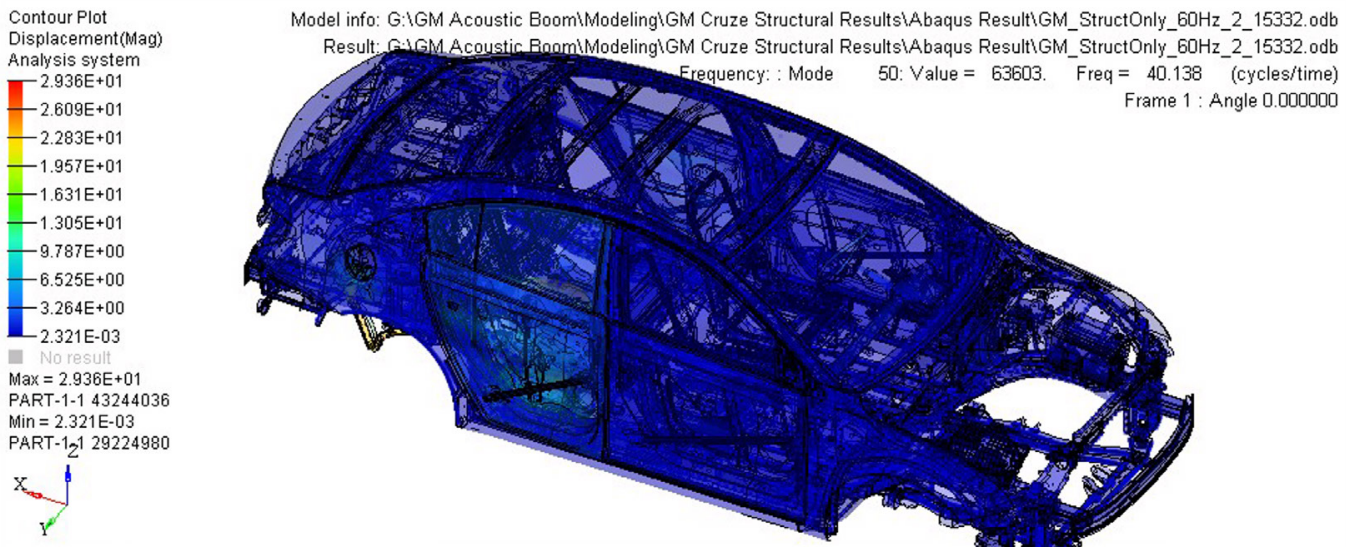

Figure 6: Abaqus structural mesh mode shape at $40.1 \mathrm{~Hz}$ 


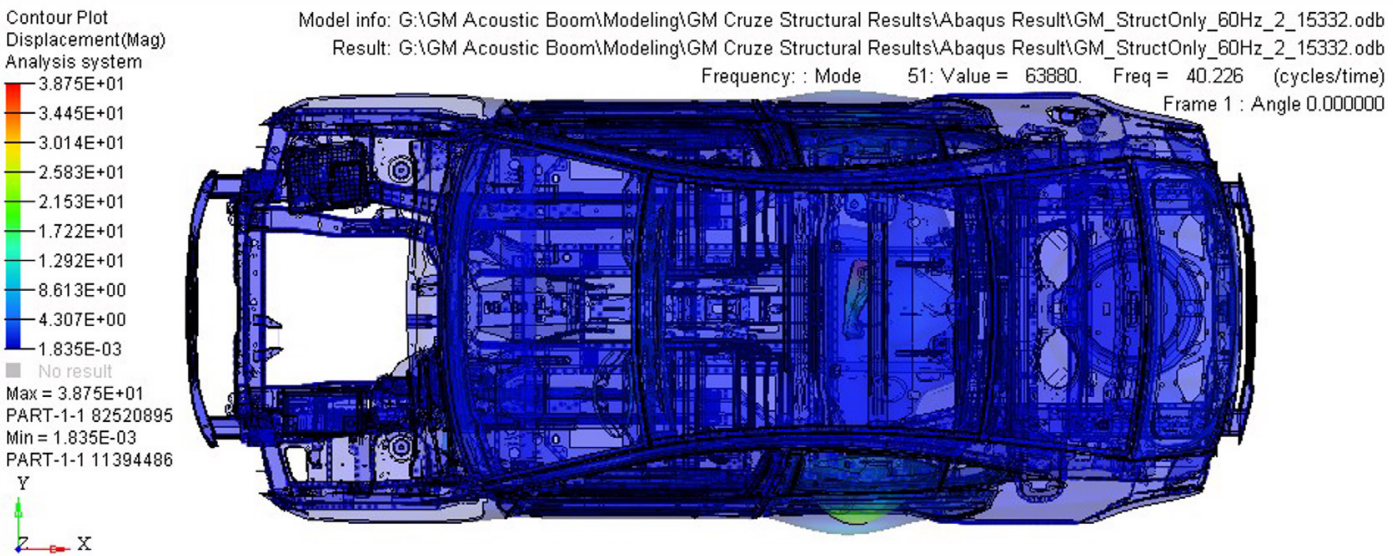

Figure 7: Abaqus structural mesh mode shape at $40.2 \mathrm{~Hz}$

Figure 6 and Figure 7 show the structural mesh mode shapes at $40.1 \mathrm{~Hz}$ and $40.2 \mathrm{~Hz}$ respectively. At $40.1 \mathrm{~Hz}$ fuel pipe has the maximum displacement along with the stiffener at the floor of the car. At $40.23 \mathrm{~Hz}$ the rear doors are out of phase with each other with the rear door behind the driver showing the maximum displacement.

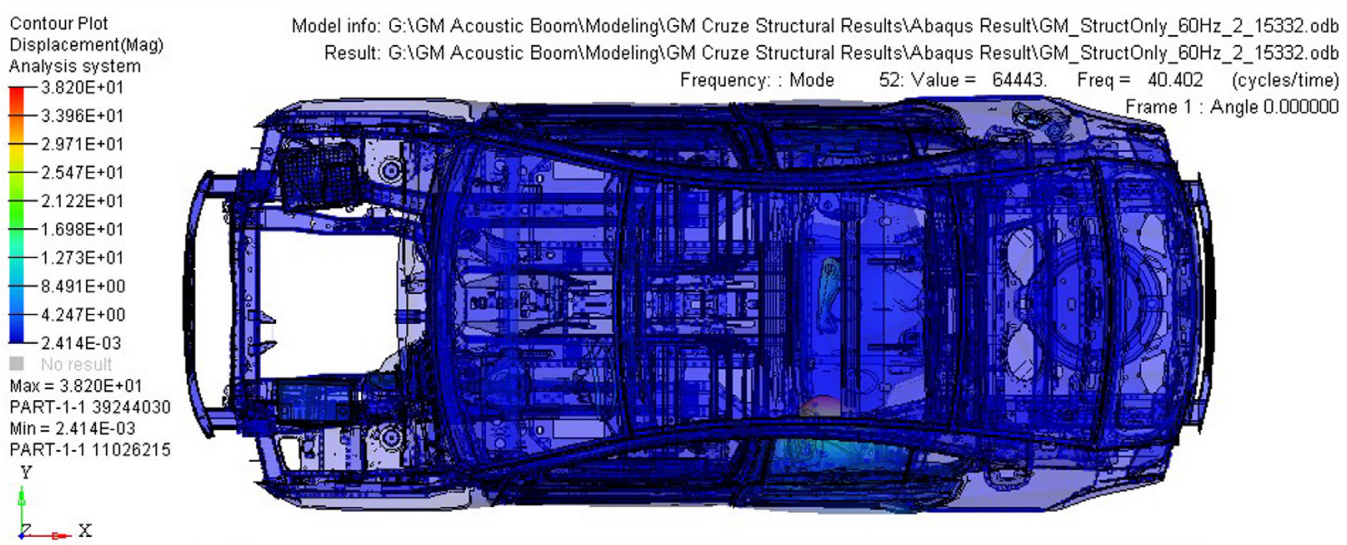

Figure 8: Abaqus structural mesh mode shape at $40.4 \mathrm{~Hz}$
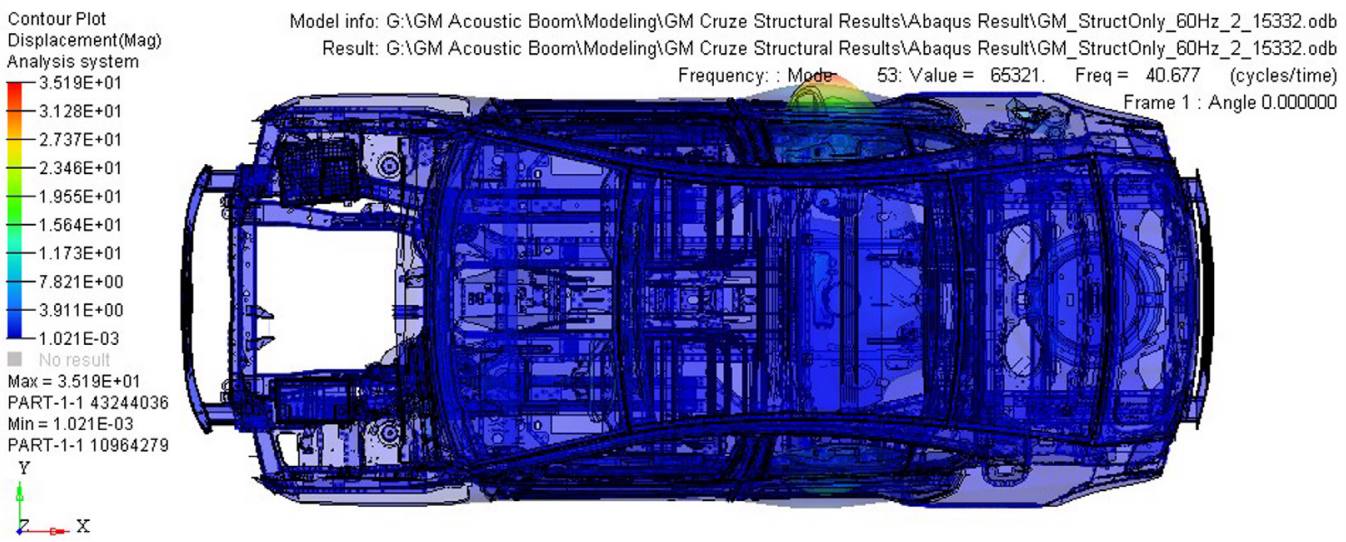

Figure 9: Abaqus structural mesh mode shape at $40.6 \mathrm{~Hz}$ 
Figure 8 and Figure 9 show the structural mode shapes at $40.4 \mathrm{~Hz}$ and $40.6 \mathrm{~Hz}$ respectively. At $40.4 \mathrm{~Hz}$ we have the rear doors going in-phase, with the rear door behind the driver having the maximum displacement. At $40.6 \mathrm{~Hz}$ we have the rear door going out-of-phase with the maximum displacement at rear door behind the passenger. Thus, acoustic boom can be caused by the mode shapes at $40.2 \mathrm{~Hz}$ and $40.6 \mathrm{~Hz}$ which squeeze the air inside thus causing a volume change inside the acoustic cavity.

Now, the mode shapes obtained from Abaqus and Optistruct were compared using the modal assurance criteria (MAC). MAC is calculated between GM Optistruct and Abaqus result using noise and vibration simulation software, LMS Virtual.Lab.

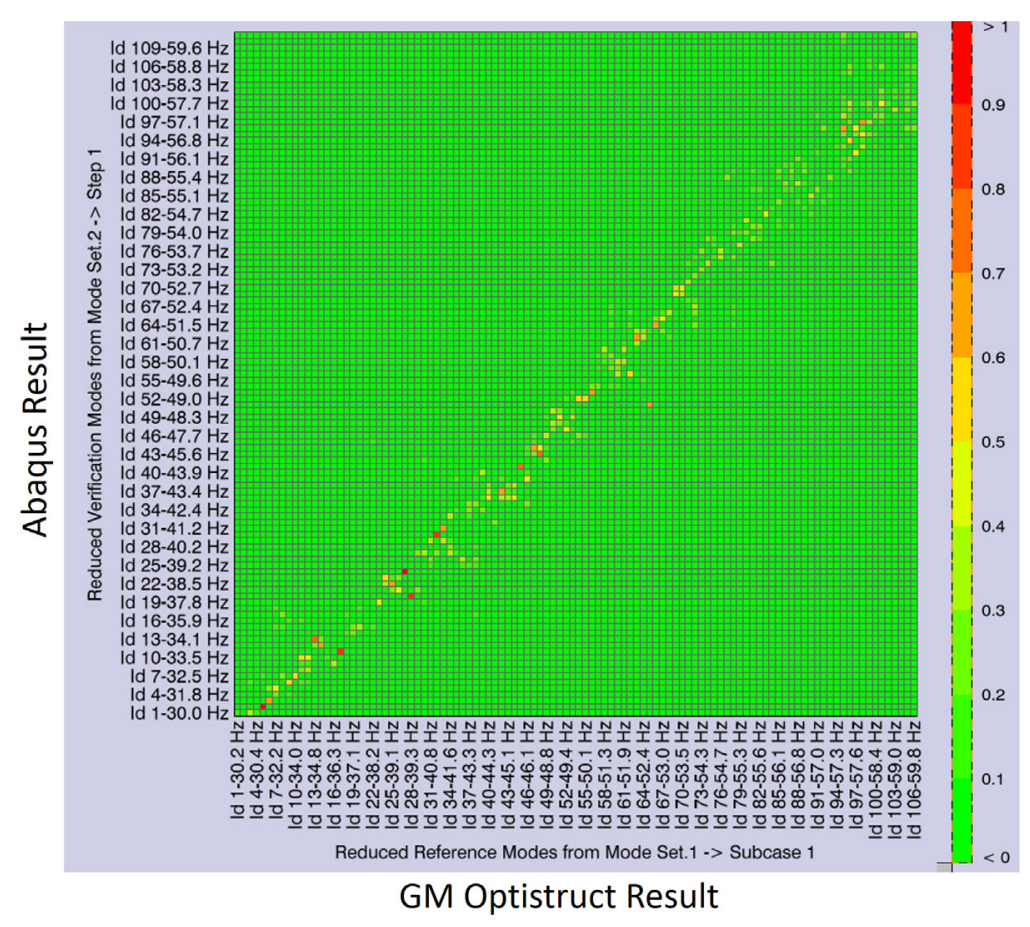

Figure 10: MAC comparison between GM Optistruct and Abaqus result 


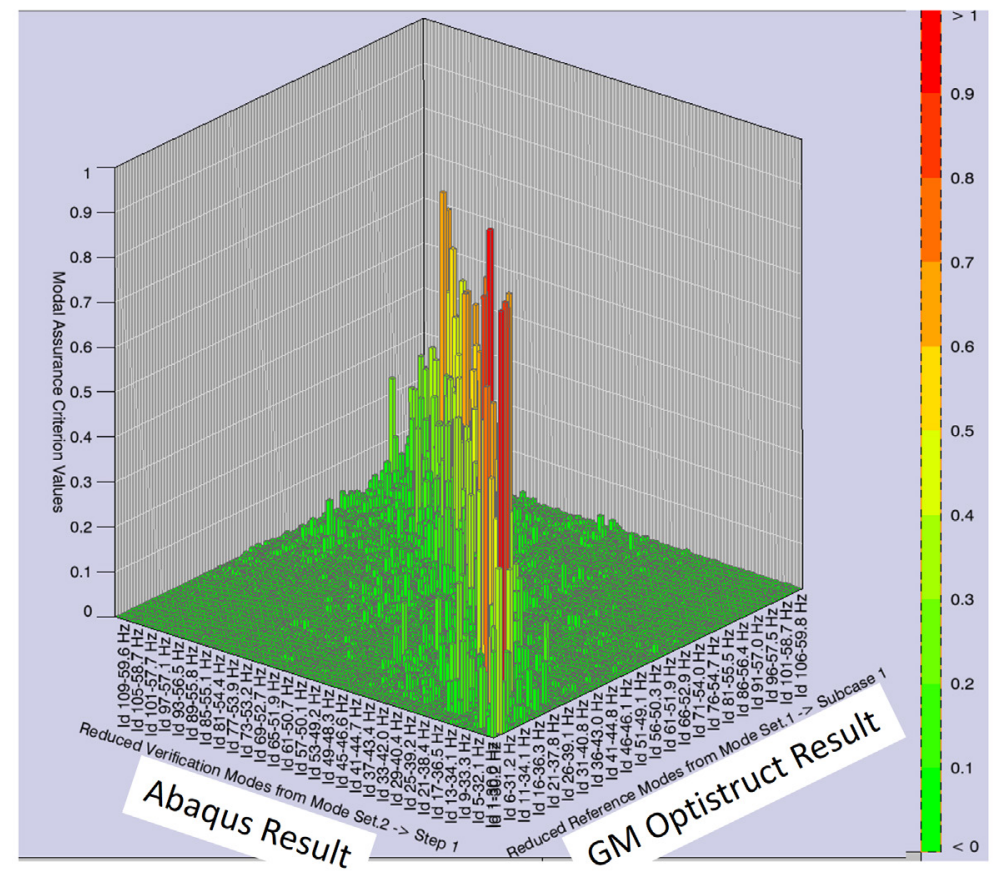

Figure 11: Isometric view of MAC comparison between GM Optistruct and Abaqus result

Figure 10 and 11 show the modal assurance criteria between the Abaqus and GM Optistruct result. The overall MAC value is low indicating low correlation, but there is a high MAC values at lower frequencies up to $50 \mathrm{~Hz}$. Also, there is lower correlation between the modes in the boom frequency of $40 \mathrm{~Hz}$. Thus, two different solutions are obtained for the same structural mesh using the Abaqus and Optistruct solver.

\subsection{Chevrolet Cruze LTZ Acoustic Mesh}

Chevrolet Cruze LTZ acoustic mesh was provided by General Motors (GM). Figures below show the acoustic mesh characteristics.

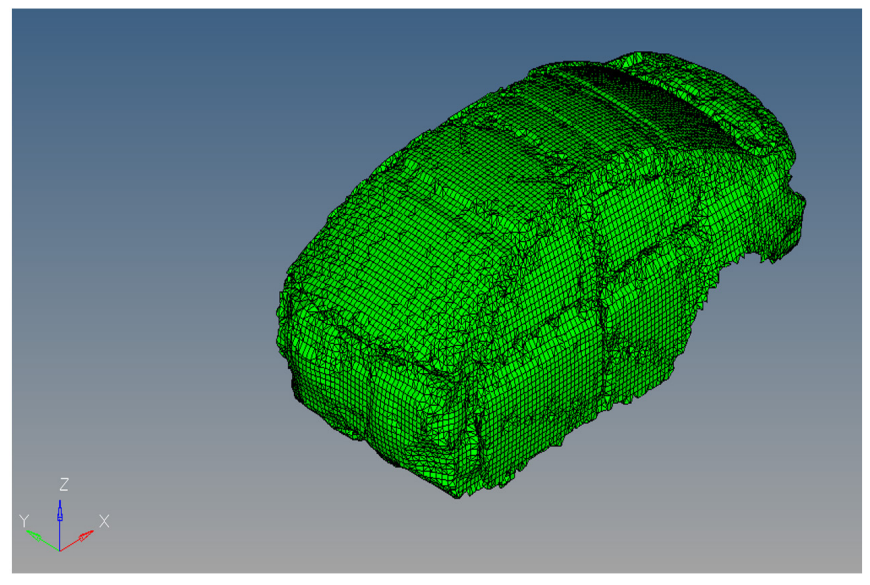

Figure 12: Cruze Acoustic Mesh 


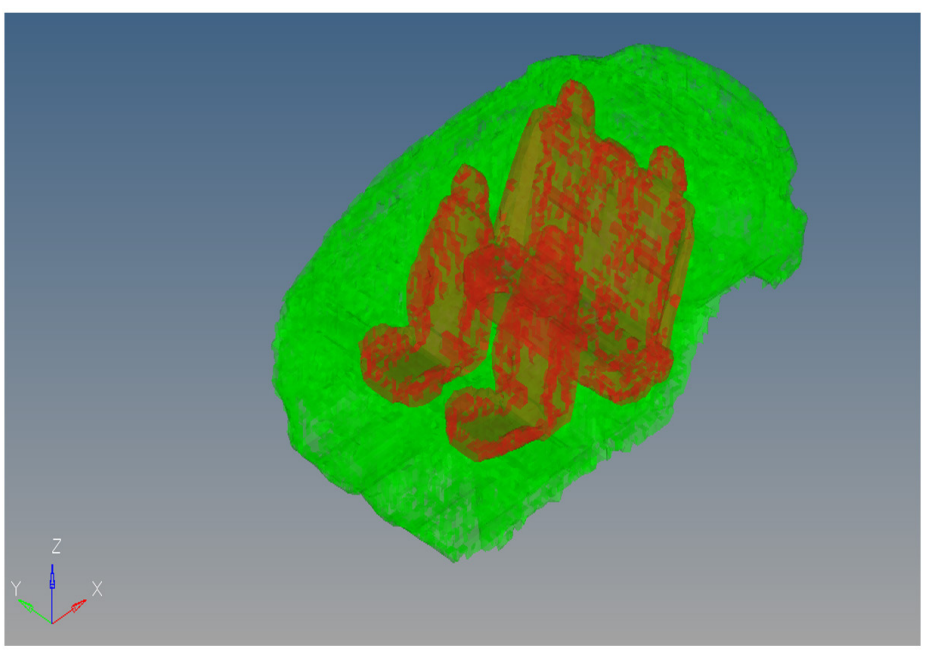

Figure 13: Component view of Cruze Acoustic Mesh showing rear seats

Figure 12 and Figure 13 show the acoustic mesh and its component view respectively. Acoustic mesh is much coarser as compared to the structural mesh because acoustic wavelengths are much shorter than structural wavelengths at low frequencies [6]. As shown, acoustic mesh is made of two separate components with the rear seats having material properties of heavy air, while the rest of cavity has material properties of air. The additional mass of rear seats accounts for structural dynamic effect of the trim [16] [17].

Two separate Acoustic meshes were provided by General Motors with differently modeled connections between rear seats and trunk. The characteristics of both the acoustic meshes are described below.

- GM Acoustic Mesh \#1: Rear Seats joined to trunk

- GM Acoustic Mesh \#2: Rear Seats separated from trunk 

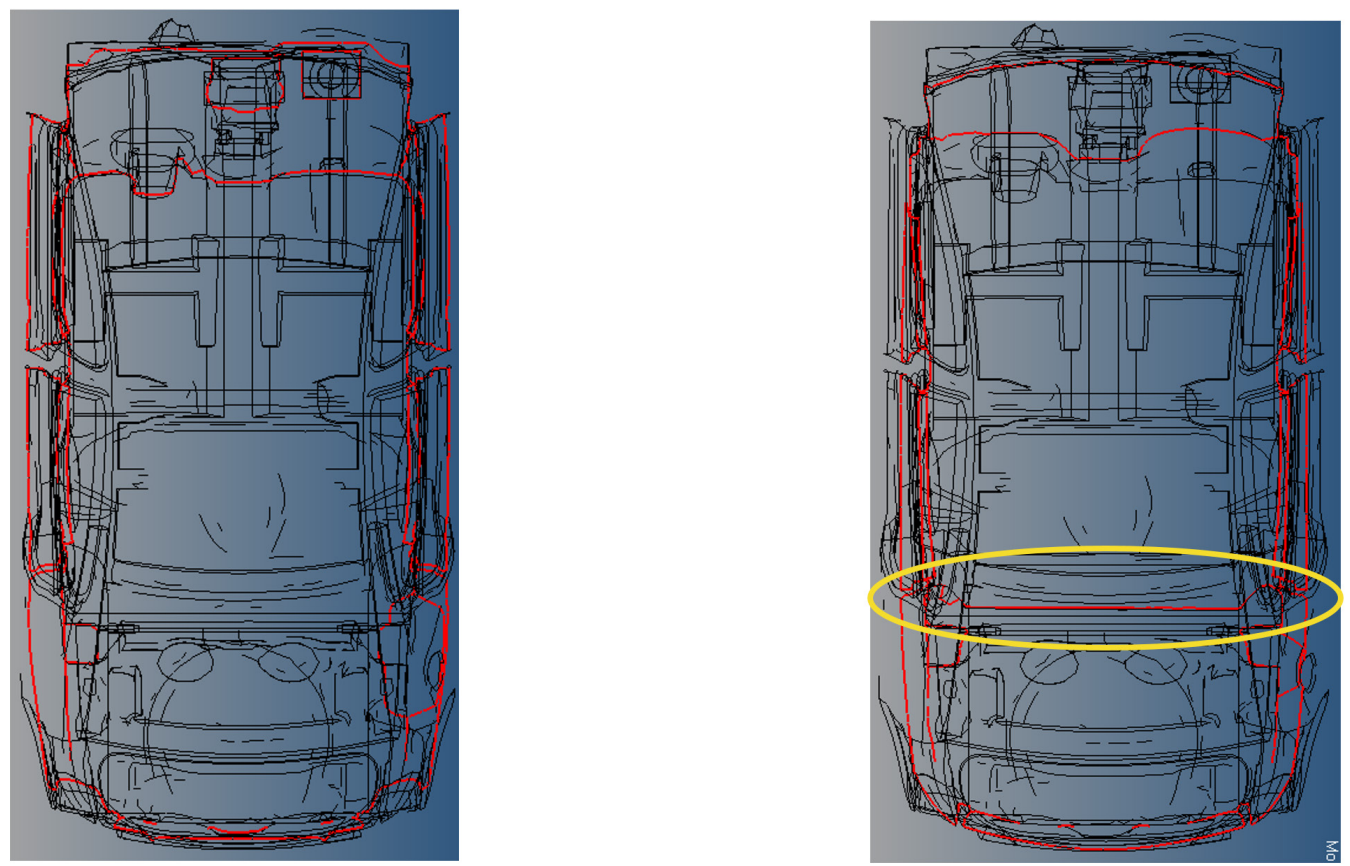

Figure 14: Top view of Acoustic Mesh \#1(left) and Acoustic Mesh \#2(right) showing differences between two meshes

Figure 14 shows the difference between acoustic mesh \#1 and acoustic mesh \#2. The figure is top view of the acoustic mesh with red lines representing surfaces. The top part of the figure is the front end and the bottom part is the rear end of the vehicle. As shown acoustic mesh \#1 has no surface or gap between the trunk and rest of cavity, whereas the trunk and rest of cavity meshes are separated in acoustic mesh \#2 by a gap. Thus, in acoustic mesh \#2, speaker holes which are represented by two circles in figure above on the rear package shelf act as connection between the trunk and rest of the cavity.

\subsubsection{Eigen solution of Acoustic Mesh \#1 \& GM Optistruct result comparison}

Cruze acoustic mesh was provided as a Nastran (.bdf) file and was converted into Abaqus (.inp) file. The converted acoustic mesh file was then solved in Abaqus and results were compared with the GM Optistruct result. 


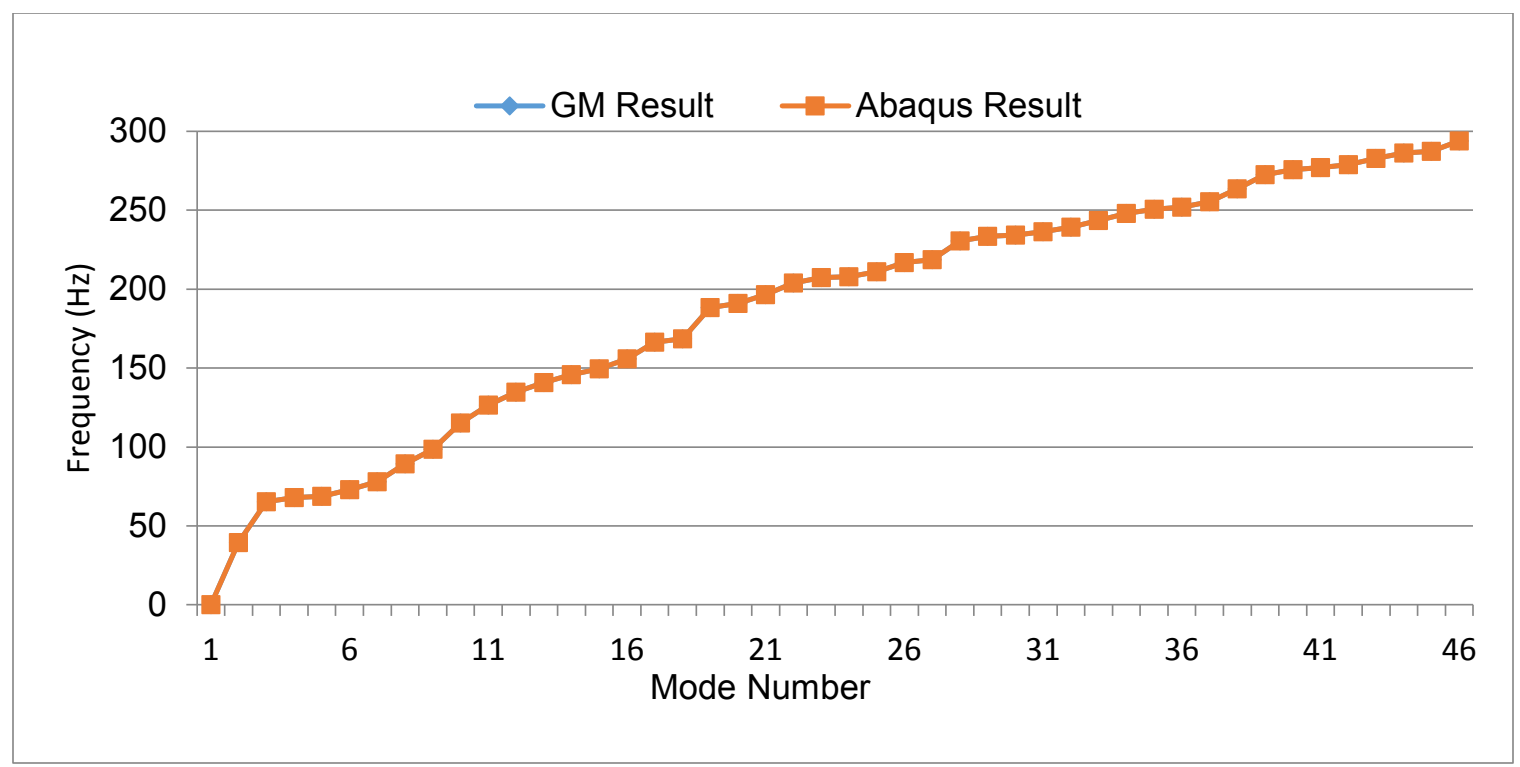

Figure 15: Natural frequencies comparison between Abaqus and GM Optistruct result for Cruze Acoustic Mesh \#1

Figure 15 shows the comparison of GM Optistruct and Abaqus result between 0 to $300 \mathrm{~Hz}$. As seen both the solvers predict exactly the same natural frequencies in the given frequency range for Acoustic Mesh \#1. Figures below show the mode shapes in the boom frequency range of $40 \mathrm{~Hz}$.
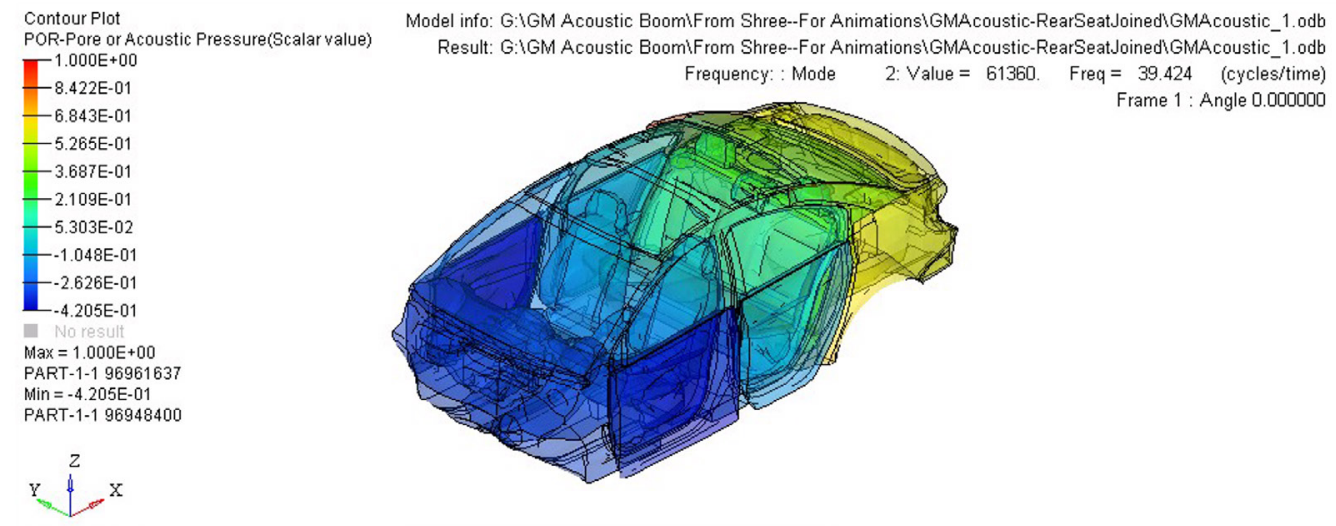

Figure 16: Mode Shape at $39 \mathrm{~Hz}$ for Acoustic Mesh \#1 

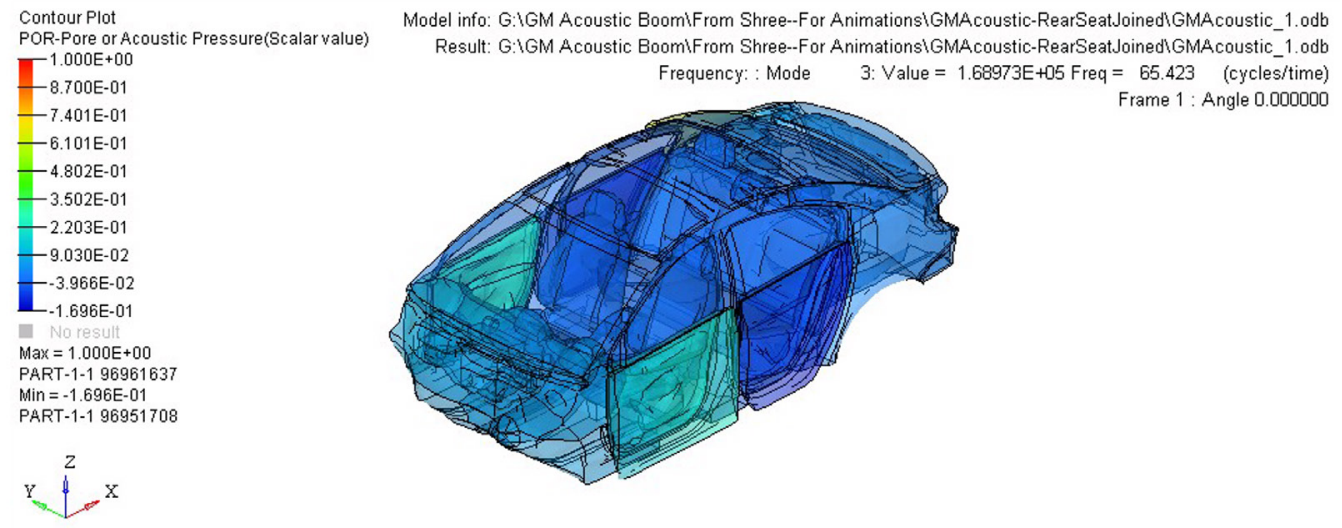

Figure 17: Mode Shapes at $65 \mathrm{~Hz}$ for Acoustic Mesh \#1

Figure 16 and Figure 17 shows mode shapes for the first flexural mode at $39 \mathrm{~Hz}$ and second flexural mode at $65 \mathrm{~Hz}$ respectively. At $39 \mathrm{~Hz}$, the acoustic sensitivity is intermittently high at the front end and trunk of the vehicle, thus the air travels from the front to the rear part of the vehicle. At $65 \mathrm{~Hz}$, the front and rear door cavities are out-of-phase and have high acoustic sensitivity.

\subsubsection{Eigen solution of Acoustic Mesh \#2 \& GM Optistruct result comparison}

Acoustic Mesh \#2 has rear seats separated from the trunk. Cruze Acoustic Mesh \#2 is imported into Abaqus and then solved for mode shape and natural frequencies.

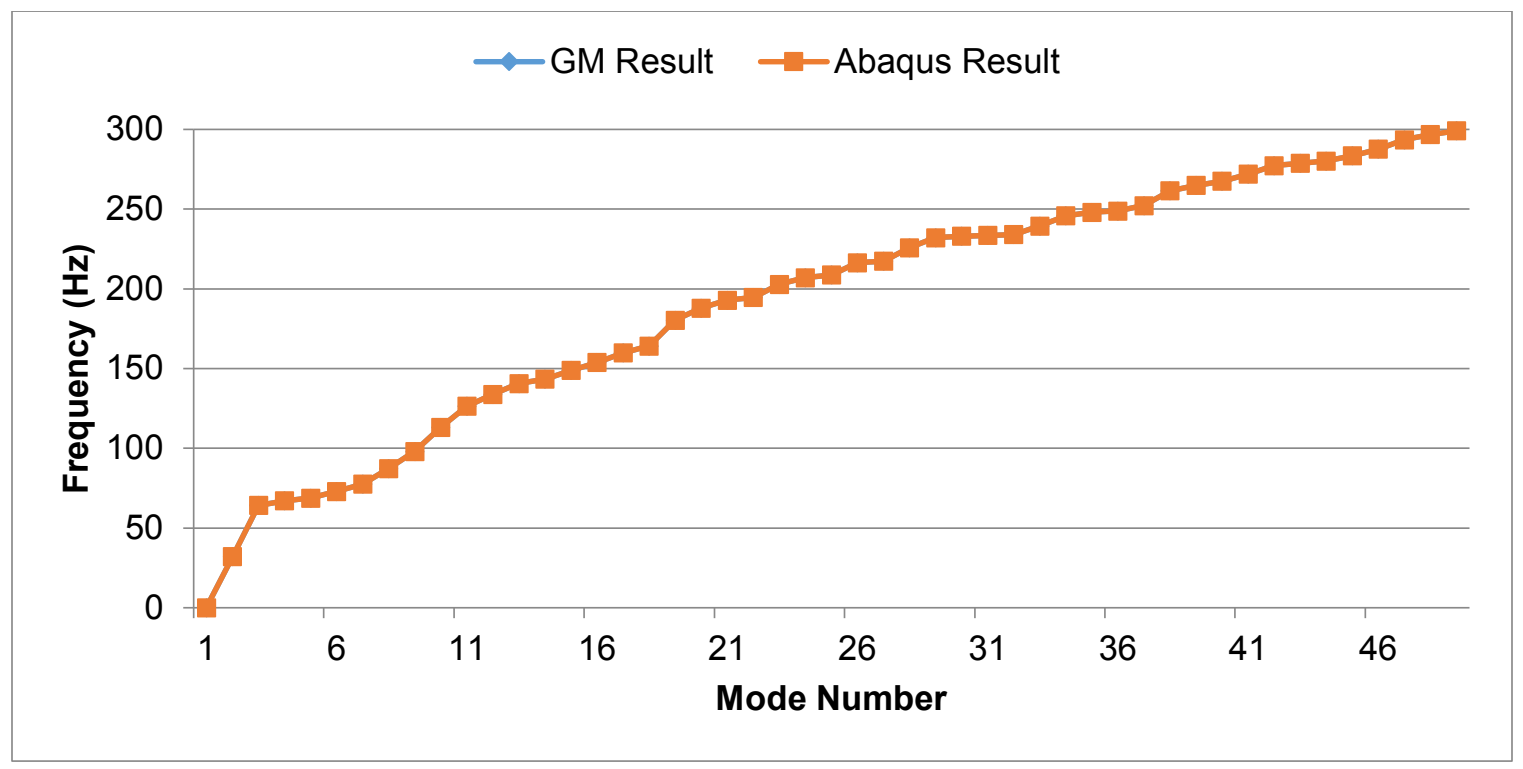

Figure 18: Natural frequencies comparison between Abaqus and GM Optistruct result of Acoustic Mesh \#2

Figure 18 shows the comparison of natural frequencies of Acoustic Mesh \#2. As seen both the graphs of overlap each other in the frequency range of 0 to $300 \mathrm{~Hz}$. 


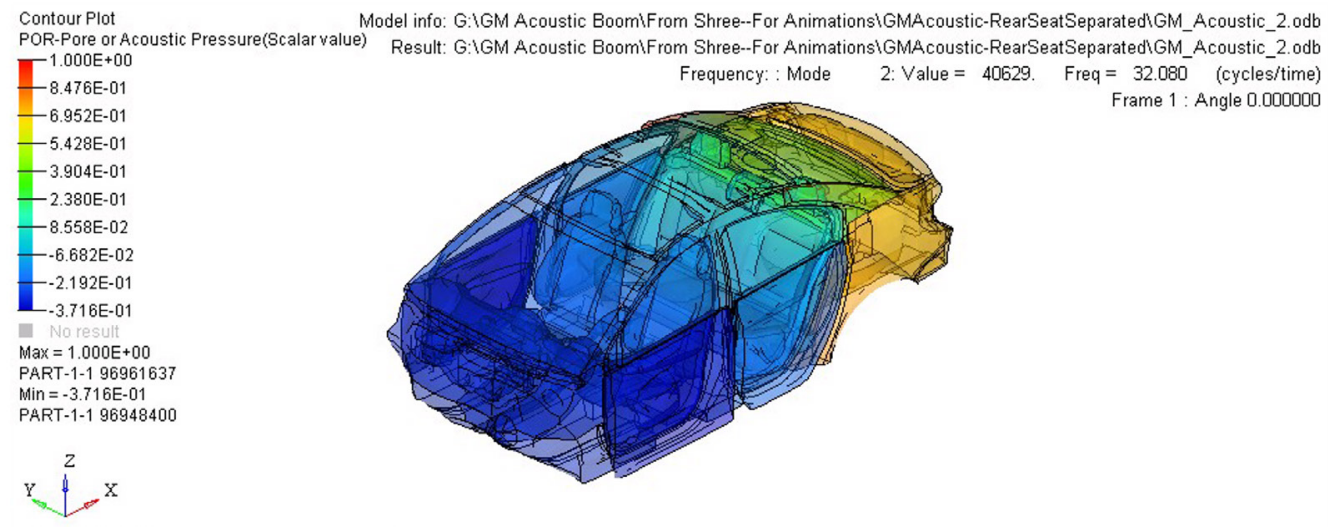

Figure 19: Mode Shape at $32 \mathrm{~Hz}$ of Acoustic Mesh \#2

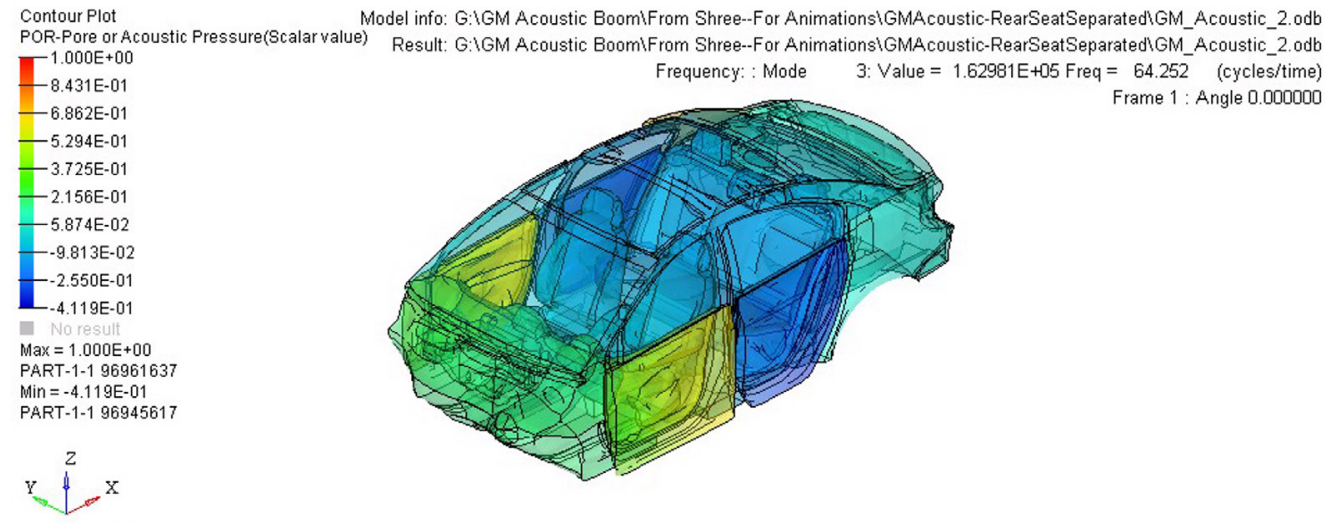

Figure 20: Mode Shape at $64.25 \mathrm{~Hz}$ of Acoustic Mesh \#2

Figure 19 and Figure 20 show the mode shapes of first two flexural natural frequencies. The first flexural mode shape at $32 \mathrm{~Hz}$ has intermittent high acoustic sensitivity from the front end to the trunk of the vehicle i.e. air travels from front end to the trunk of the vehicle and second flexural mode shape is at $64 \mathrm{~Hz}$ with the front and rear door cavities having a high acoustic sensitivity value.

\subsection{Chevrolet Cruze LTZ Coupled Solution Analysis}

Now, after individually analyzing structural mesh and acoustic mesh \#1 and acoustic mesh \#2, the structural-acoustic coupled solution is analyzed. The structural mesh is coupled with both the acoustic meshes and the results were analyzed. Thus we have:

- GM Coupled Solution \#1 - Coupled solution of GM Structural Mesh and GM Acoustic Mesh \#1

- GM Coupled Solution \#2 - Coupled solution of GM Structural Mesh and GM Acoustic Mesh \#2 


\section{a. Coupling Definition}

The Cruze Structural Mesh and Acoustic Mesh \#1 were coupled in Abaqus using 'tie constraints'. Tie constraints are applied in Abaqus by defining two coupling surfaces. According to Abaqus terminology, a master surface should have higher wave velocity as compared to the slave surface [7], thus the inside surface of a structural mesh and outer surface of acoustic mesh were defined as master and slave surfaces respectively. Various ways of defining the coupling surfaces were applied and finally two surfaces were defined that represented the interacting surfaces in the actual Chevrolet Cruze LTZ. A tolerance value of $20 \mathrm{~mm}$ was applied to the tie constraints, which means all the acoustic nodes in the $20 \mathrm{~mm}$ diameter range of a structural node are tied with the structural or master node. Tie constraints are single degree of freedom elements that tie two surfaces together so that the displacements or pressures of first surface are applied on the second surface and vice versa. Care was taken that all the major structural and acoustic mesh surfaces like seats, door cavities and trunk are coupled. Although, some elements are missing intersection between the GM structural solution and Acoustic Mesh \#1 but they were ignored as they don't have a large surface area for coupling.

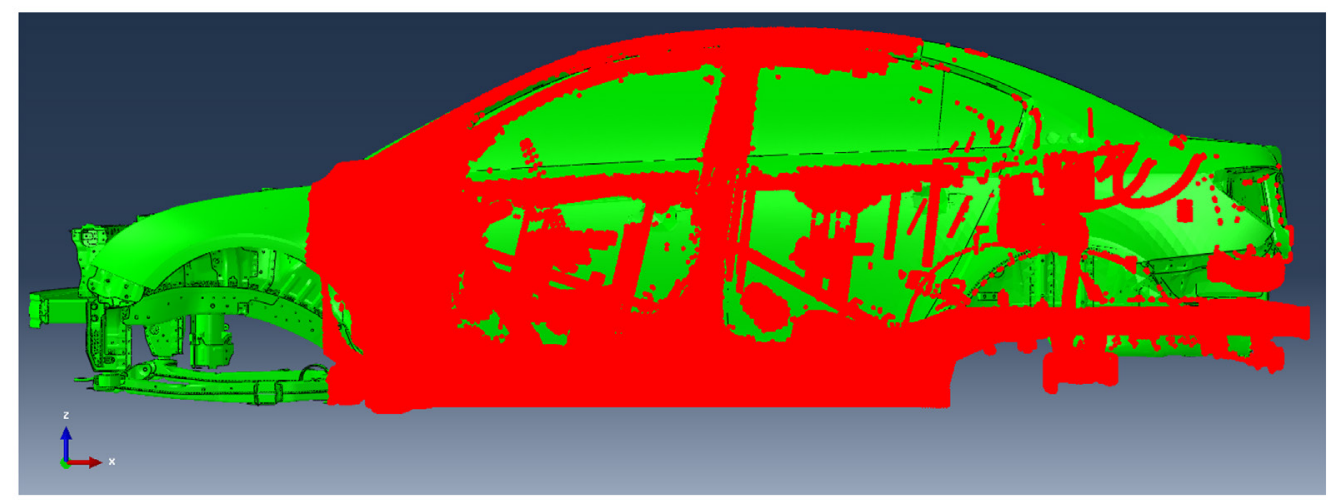

Figure 21: Nodes missing intersection represented by red between the coupling surfaces

Figure 21 shows the nodes missing intersection between the GM Structural mesh and GM Acoustic Mesh \#1. As shown, the bottom plate of the car was not connected as it lies on the outer surface of the structural mesh. Also, steering wheel and small components inside the door cavities were not coupled as they are not adequately modeled in the acoustic mesh i.e. there is no cavity for these components in the acoustic mesh for the corresponding structural mesh. Similarly coupling method was applied for coupled solution \#2.

\section{b. FRF calculation in Abaqus}

Full structural modal test was performed on the Cruze LTZ model with two shaker inputs and various output points on the vehicle chassis. To simulate the test results, frequency response function were calculated on the coupled structural-acoustic mesh using the same steps as specified before. 


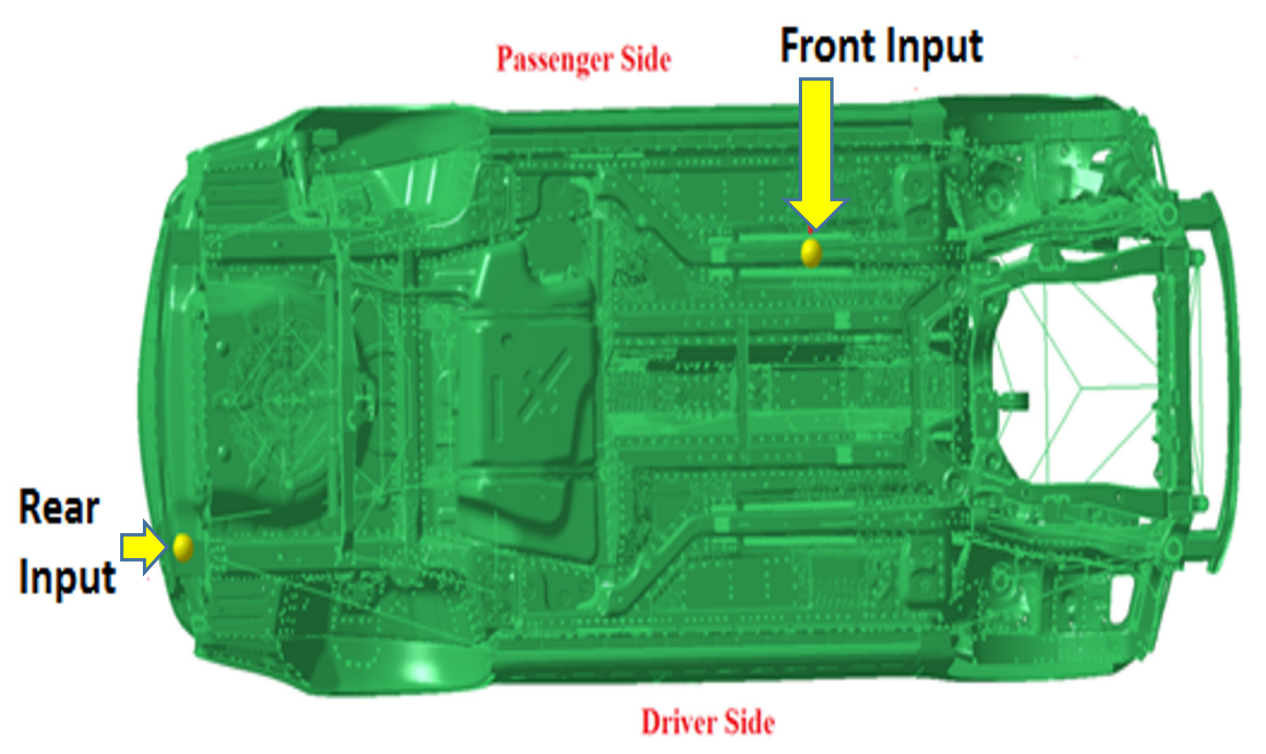

Figure 22: Structural input points on Cruze model for FRF generation

Figure 22 shows the input point location on the structural mesh. The output points for the FRF are defined at different points on the structural and acoustic mesh, so that they include all the major components of the passenger car.

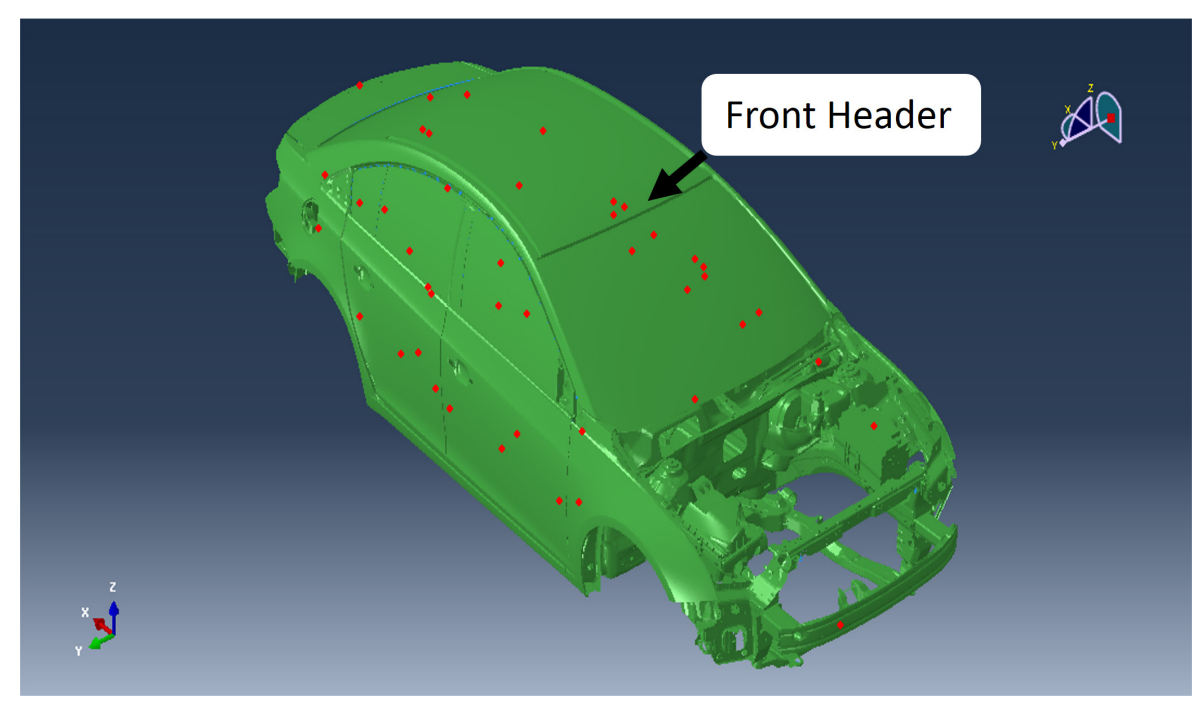

Figure 23: Structural output points on Cruze model for FRF generation

Figure 23 shows the output points represented by red dots for FRF generation. The output points are located on the outer surface and also located on various points like the rear seats, steering wheel etc. inside the car. Solution for the above model was calculated in the frequency range of $10 \mathrm{~Hz}-60 \mathrm{~Hz}$ with frequency resolution of $0.1 \mathrm{~Hz}$. Unit force was applied on the mesh in the $\mathrm{Y}$ direction at the input points. All the subsequent response is evaluated without any applied modal damping. 


\subsubsection{Coupled Solution \#1 Analysis}

\section{a. Eigen Solution of Cruze Coupled Solution \#1}

The natural frequencies of the Cruze coupled solution\#1 are calculated and compared to the Cruze structure only solution.

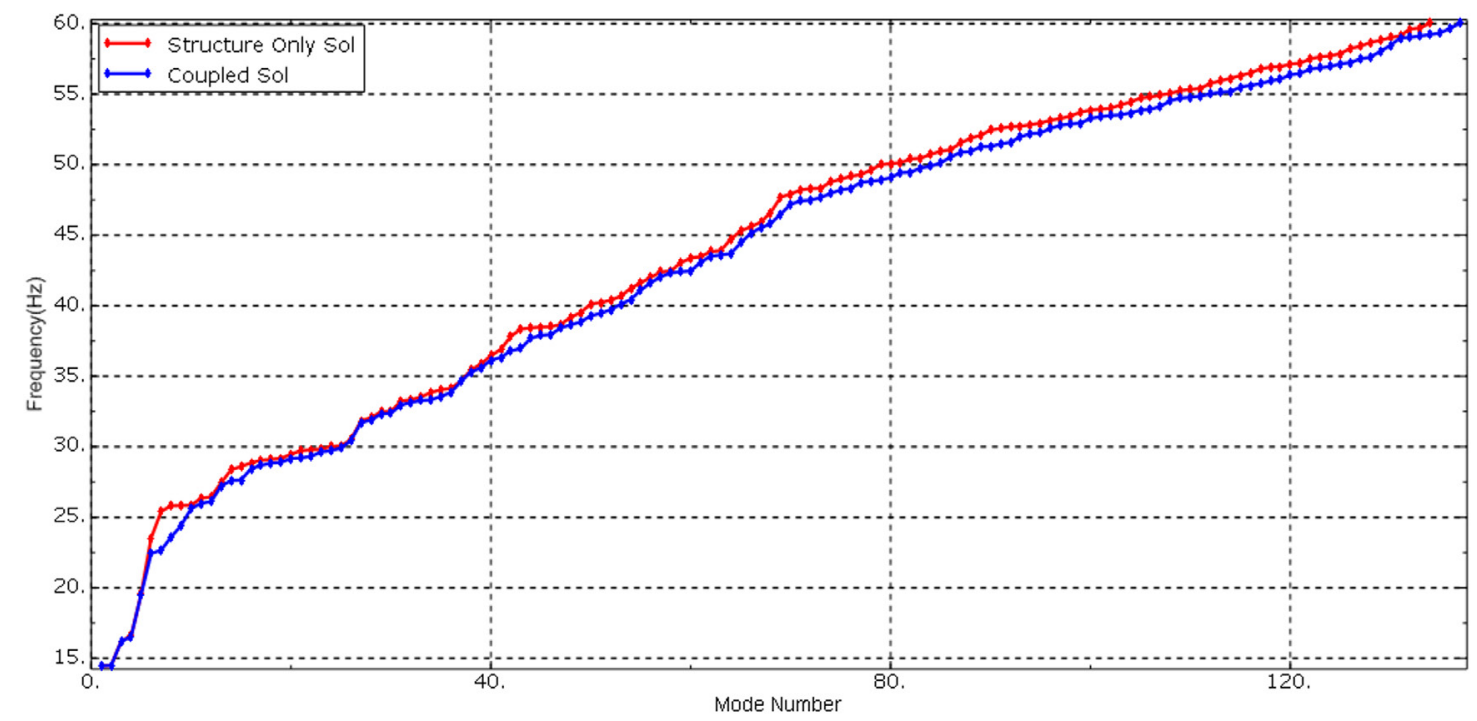

Figure 24: Natural frequency comparison between Structure Only and Coupled Solution\#1

Figure 24 shows the natural frequency of the coupled solution\#1 and structure only solution in the range of $10 \mathrm{~Hz}$ to $60 \mathrm{~Hz}$. As shown, the coupled solution has lower natural frequency throughout the frequency range as compared to the structure only solution due to the added mass of the Acoustic mesh \#1. Mode shapes of the acoustic-structural mesh were also extracted and are shown below. Due to unique modeling capabilities of Abaqus, mode shapes of structural and acoustic components in the coupling can be viewed and analyzed separately. Mode shapes on left are the coupled structural mesh mode shapes and on right are the corresponding coupled acoustic mode shapes. Structural mode shapes are shown with zero displacement represented by green, negative displacement represented by black and positive displacement represented by red. For the acoustic mode shapes the variation of acoustic pressure is shown with the zero acoustic pressure represented by green and the negative and positive maximum acoustic pressures represented by black and red respectively. 

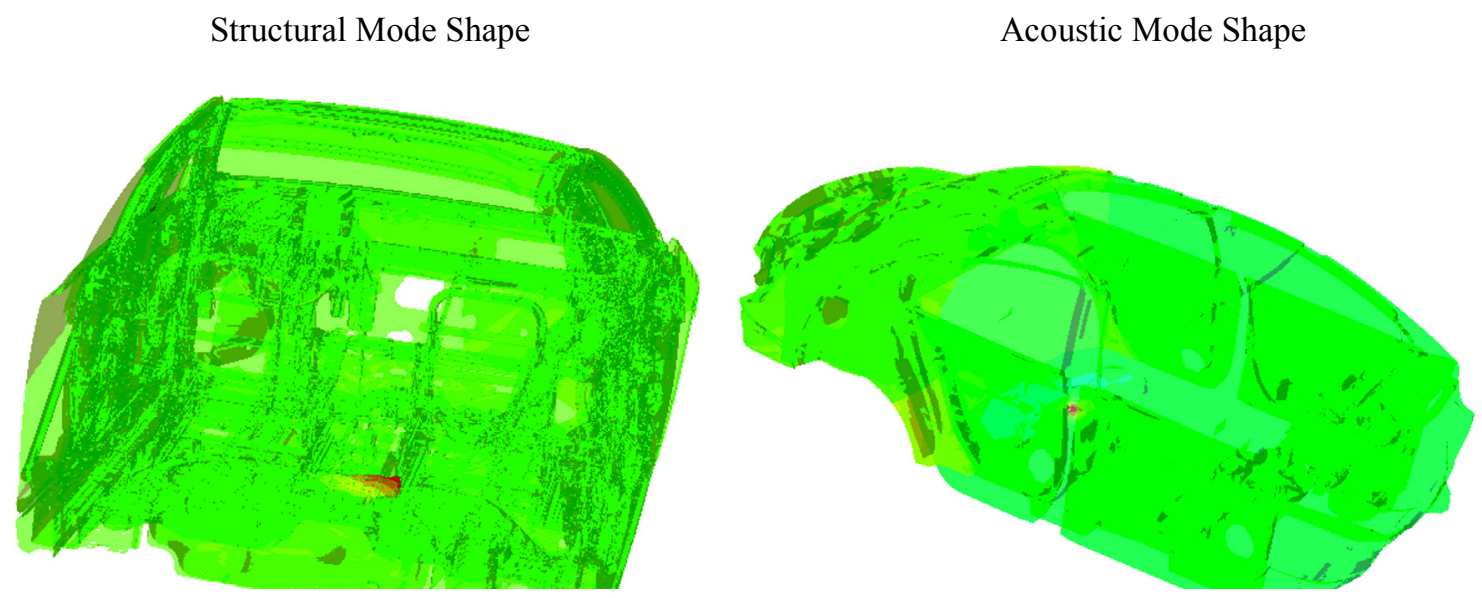

Figure 25: Coupled Mode Shapes at 39.7 Hz for Coupled Solution \#1

Figure 25 shows the coupled mode shapes for Coupled Solution \#1. For the coupled structural mode at $39.7 \mathrm{~Hz}$ a part from the floor of the vehicle is moving vertically as shown which is similar to the GM structure only solution. Similarly, on the acoustic mesh, there is high acoustic pressure at the same point due to the structural mesh pushing on the acoustic mesh.

Structural Mode Shape

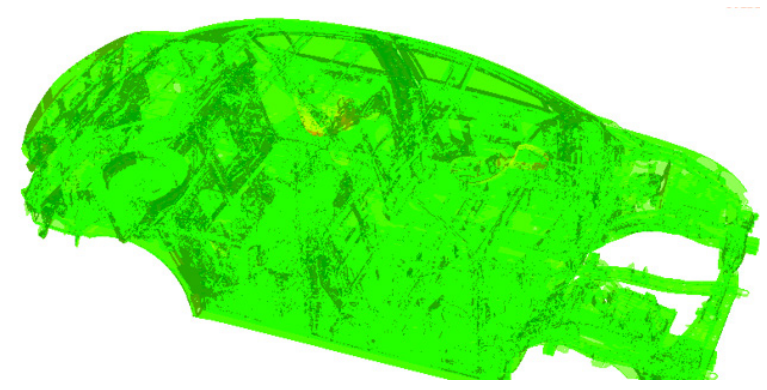

Acoustic Mode Shape

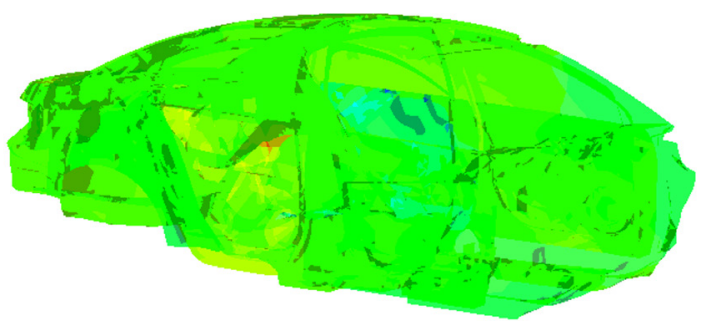

Figure 26: Coupled Mode Shapes at $41.1 \mathrm{~Hz}$ for GM Coupled Solution \#1

Figure 26 shows rear doors going out-of phase in the structural mesh, thus squeezing the acoustic mesh. Acoustic mesh also shows rear doors having a higher acoustic sensitivity which is similar to the second pure acoustic mode.

\section{b. FRF calculation for Coupled Solution\#1}

Now, FRF's are calculated at various points on structural and acoustic mesh of the coupled solution to find the response at various components of the structural-acoustic coupled system due to structural inputs. The structural and acoustic output points are chosen according to the accelerometer and microphone locations during the full structural modal, full acoustic modal test and door cavity testing. 


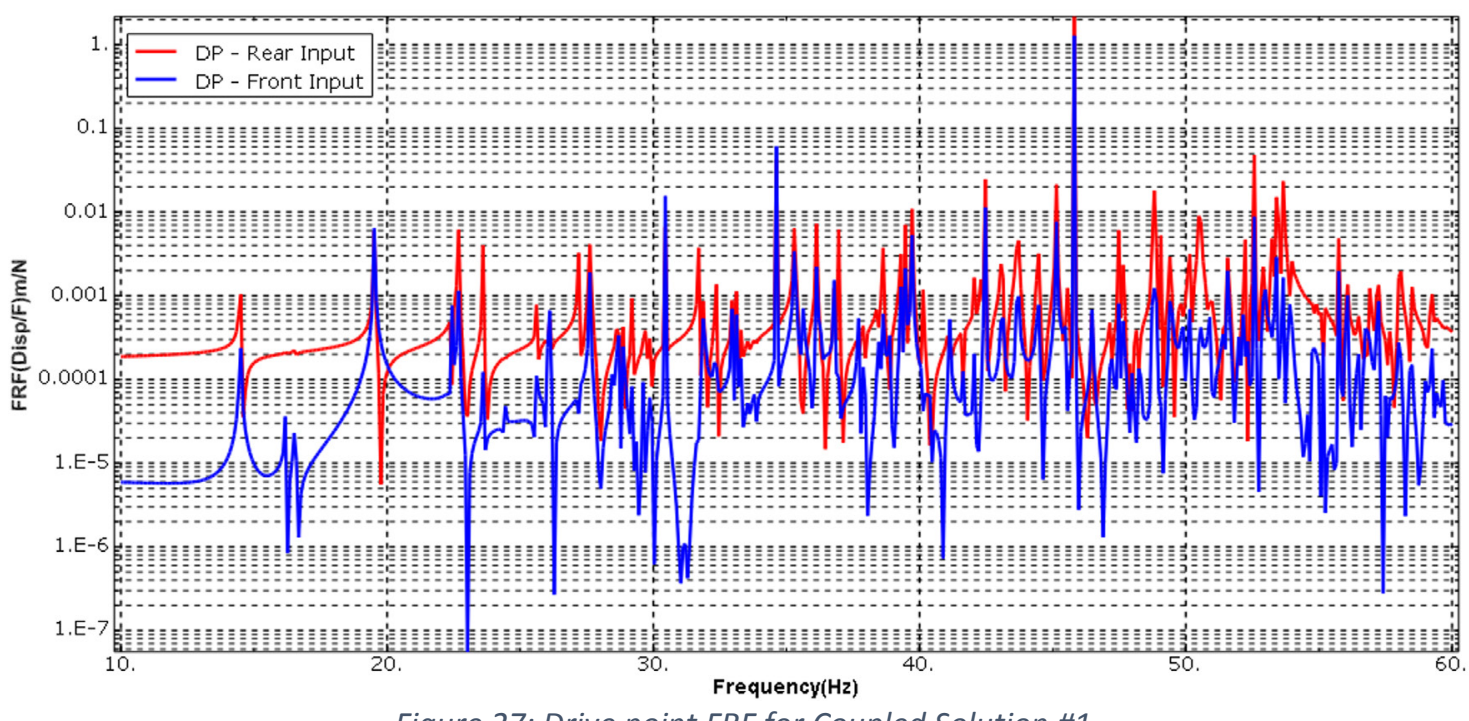

Figure 27: Drive point FRF for Coupled Solution \#1

Figure 27 shows the drive point FRF's due to the front and rear inputs for coupled solution \#1. As shown the rear input response is higher than the front input response throughout the frequency range.

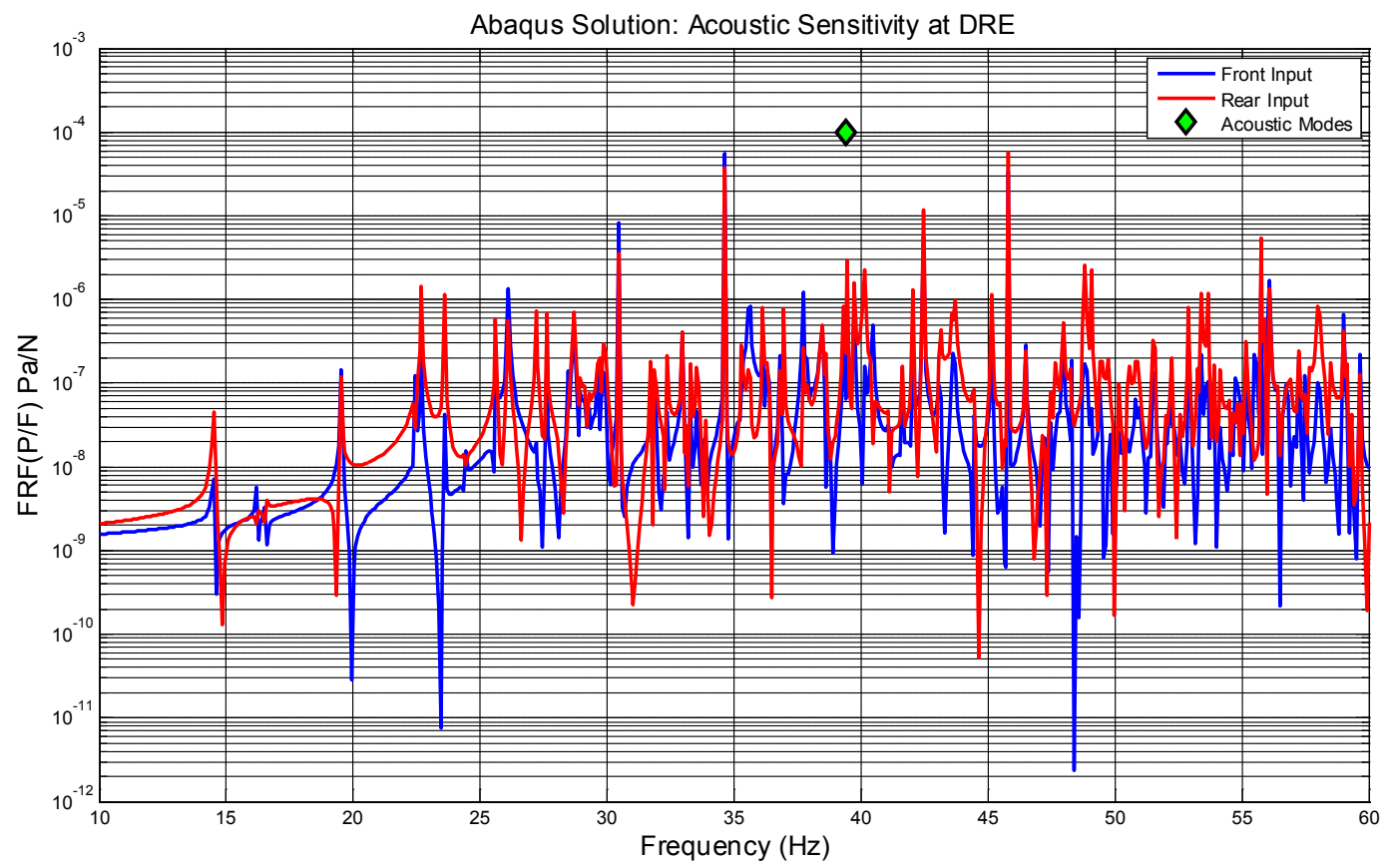

Figure 28: Comparison of acoustic sensitivity at DRE for Coupled Solution \#1

Figure 28 shows the acoustic sensitivity at driver's right ear (DRE). Acoustic sensitivity at the driver's right ear is an automotive industry standard to measure the acoustic sensitivity inside the car cabin. As shown, acoustic boom at DRE is observed at $35 \mathrm{~Hz}$ and $45 \mathrm{~Hz}$. The 
green spade shaped marker represents the pure acoustic mode at $38 \mathrm{~Hz}$. Many components like the door cavities, rear seats, trunk etc. have a large hollow volume and had higher acoustic sensitivities during modal testing. So, FRF's were also evaluated at these locations.

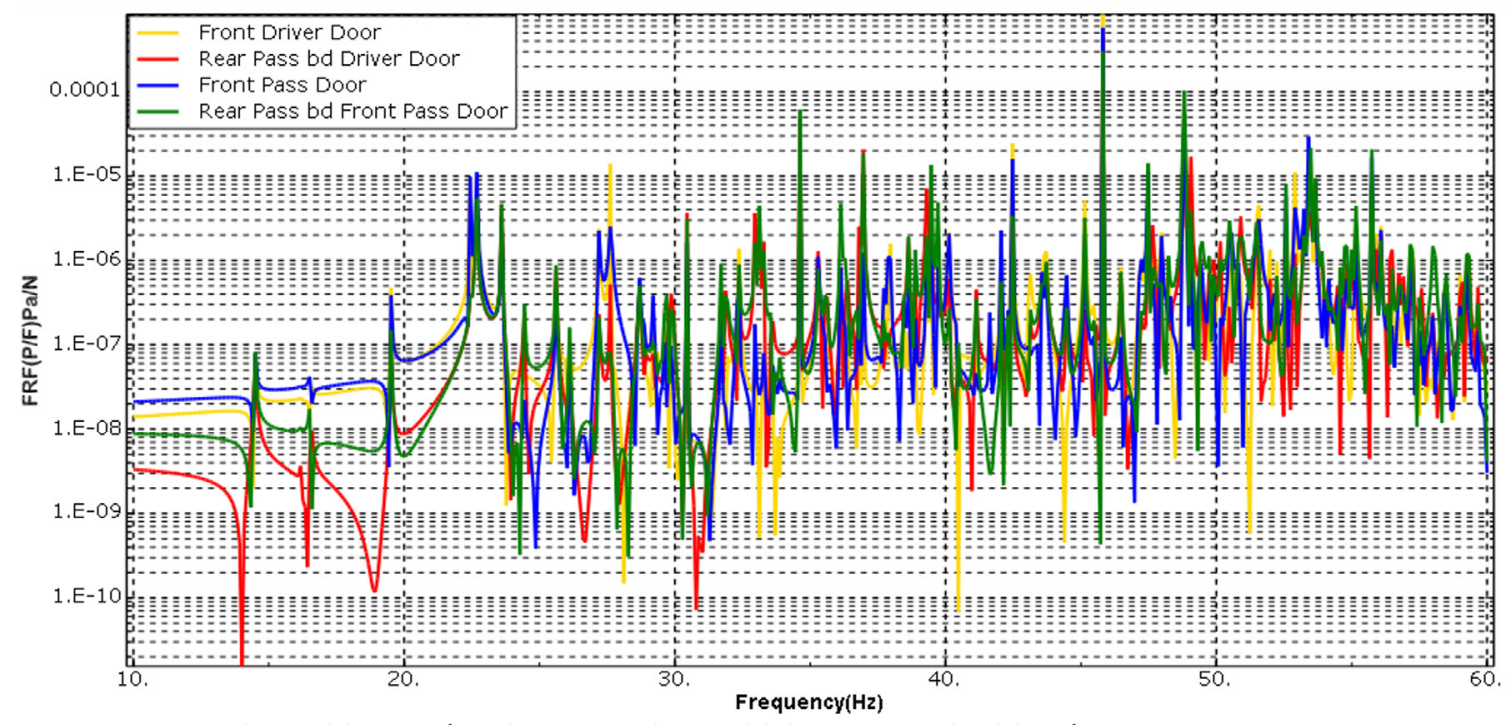

Figure 29: FRF showing acoustic sensitivity at Door Cavities due to Rear Input

Figure 29 shows the acoustic sensitivity at door cavities due to rear input. Here, all the four door cavities have a high acoustic sensitivity around the boom frequency of $40 \mathrm{~Hz}$.

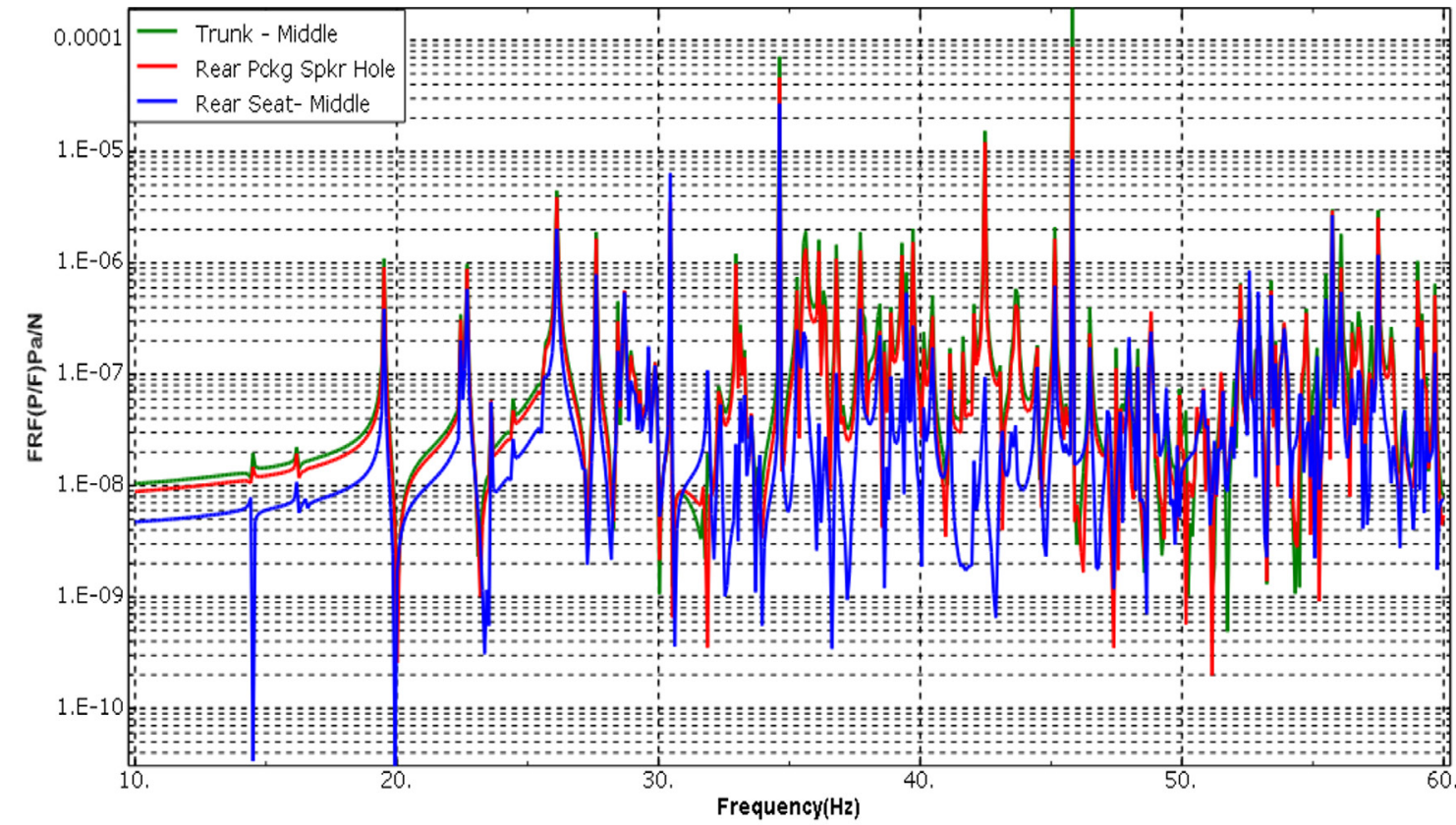

Figure 30: FRF showing acoustic sensitivity at different points due to Front Input

Figure 30 shows the acoustic sensitivity at trunk and rear package speaker hole and rear seats due to the front input. As seen the rear seat acts as an acoustic barrier and thus has a lower acoustic sensitivity throughout the frequency range with the rear package and 
speaker hole having the same magnitude from $10 \mathrm{~Hz}$ to $60 \mathrm{~Hz}$. Now, the structural and acoustic meshes are compared using frequency response functions (FRF's) with the same input and output points to evaluate the effect of addition of acoustic mesh.

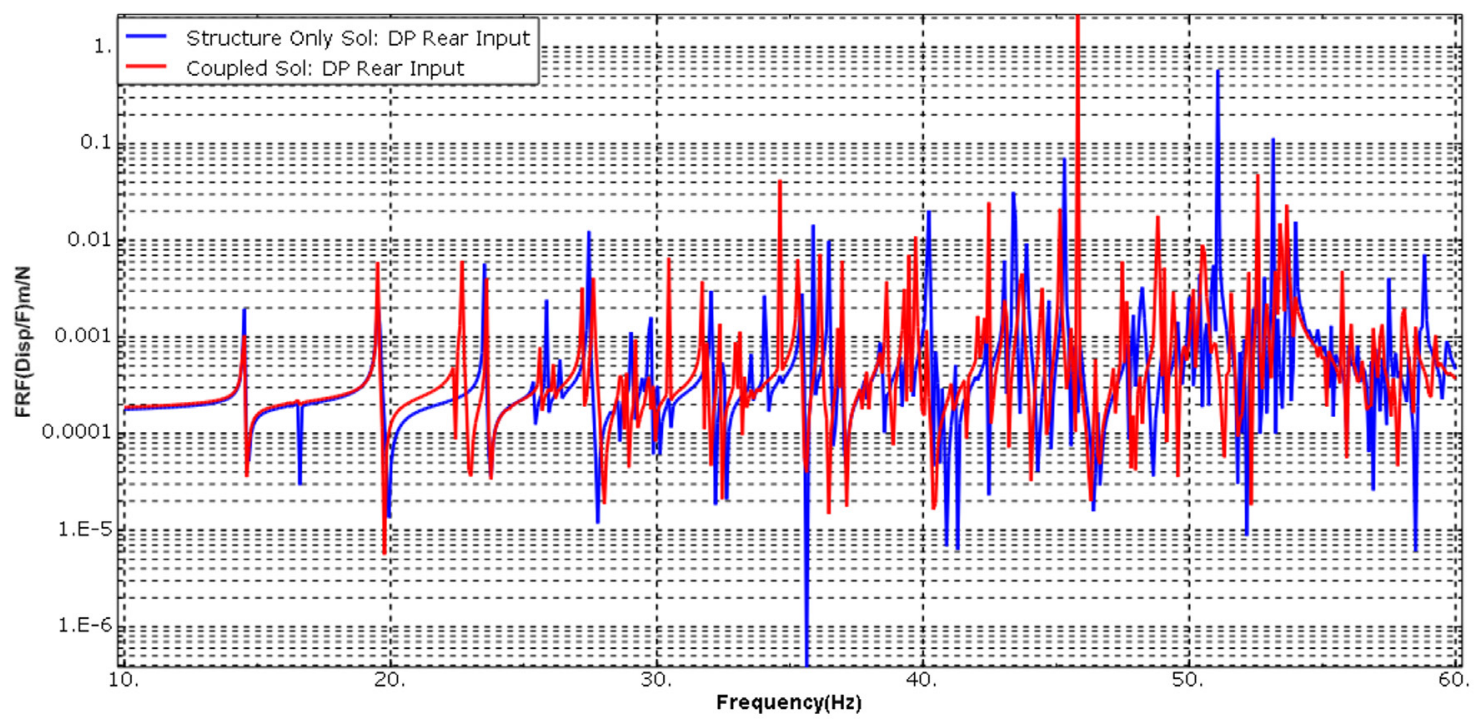

Figure 31: Drive point comparison between Structure Only Solution and Coupled Solution \#1 due to rear input

Figure 31 shows the drive point comparison between structure only solution and Coupled Solution \#1. As shown although they follow the same trend, new frequencies are introduced and also frequency shifts occur due to addition of acoustic mesh.

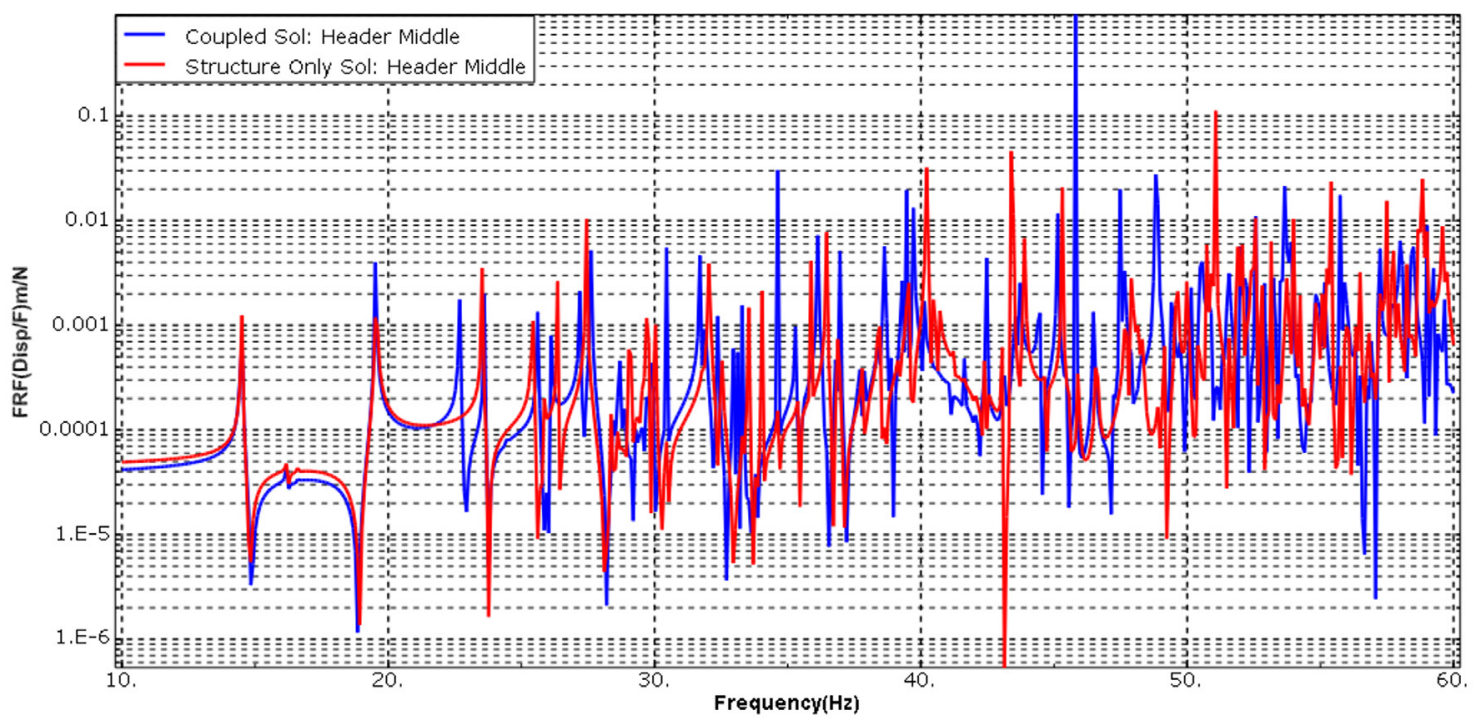

Figure 32: Comparison of Structure Only Solution and Coupled Solution \#1 for output at Header Middle due to rear input

Figure 32 shows the comparison between Structural only solution and Coupled solution\#1 due to the rear input. We have a coupled solution \#1 output at $35 \mathrm{~Hz}$ as compared to high structural output at $33 \mathrm{~Hz}$. 


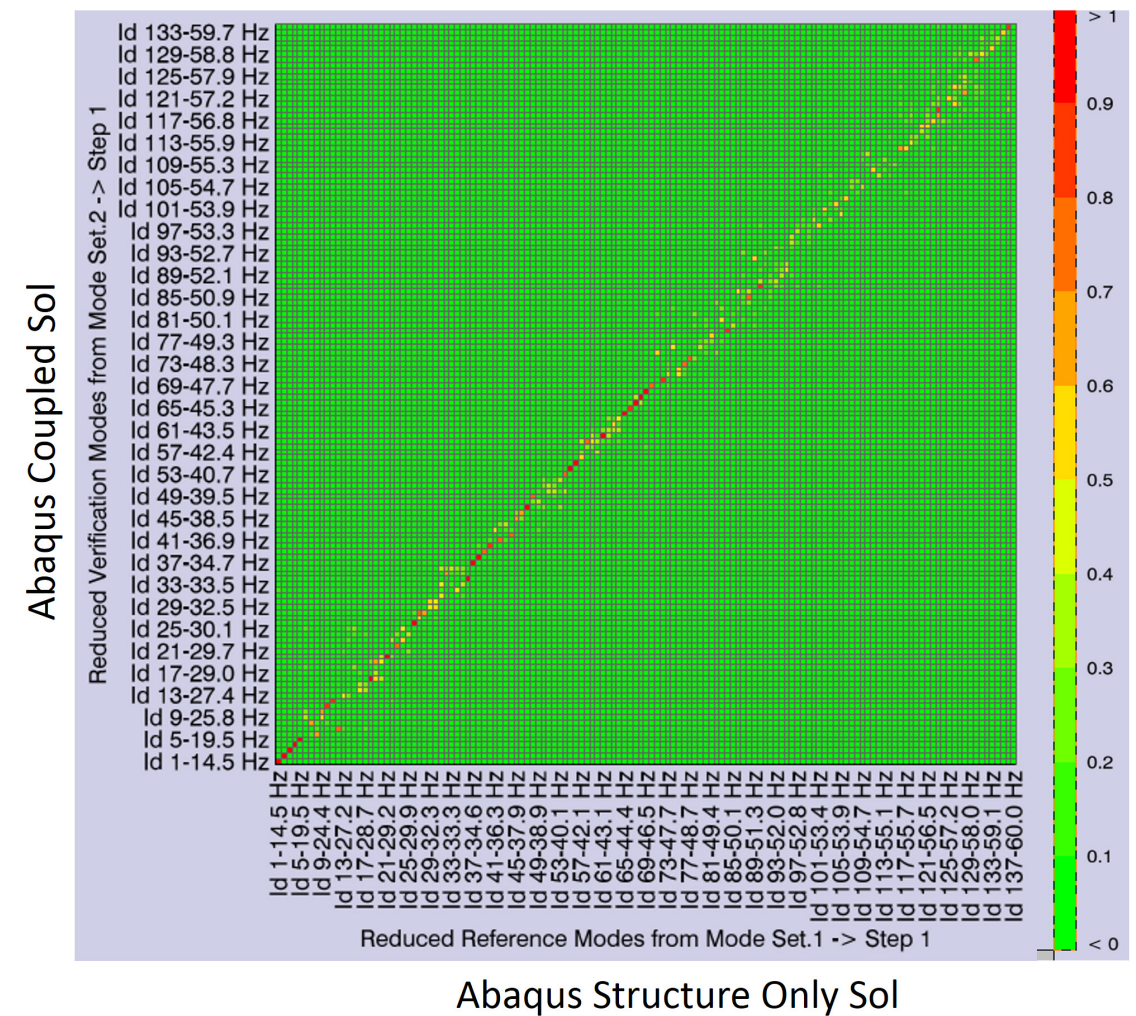

Figure 33: Modal Assurance Criteria between Coupled Structural Solution \#1 and Structure Only Solution

Figure 33 shows the MAC between the Coupled Solution\#1 and Structural Only Solution using LMS Virtual. Lab. As shown, we have a diagonal MAC with high MAC values of 0.9 up to $50 \mathrm{~Hz}$ with a non-diagonal MAC plot after $50 \mathrm{~Hz}$. At $29 \mathrm{~Hz}, 40 \mathrm{~Hz}$ and $43 \mathrm{~Hz}$ we have lower MAC values in the range of 0.5 to 0.7 , indicating the influence of acoustic modes at these frequencies. Thus, at the boom frequency of $40 \mathrm{~Hz}$ the corresponding pure acoustic mode shapes influence the coupled mode shapes.

\subsubsection{Cruze Coupled Solution \#2}

\section{a. Eigen Solution of Cruze Coupled Solution \#2}

Cruze Structural Mesh and Acoustic Mesh \#2 were coupled together using tie constraints in Abaqus. A methodology similar to acoustic mesh \#1 was employed during the acousticstructural coupling of structural mesh and acoustic mesh \#2. 


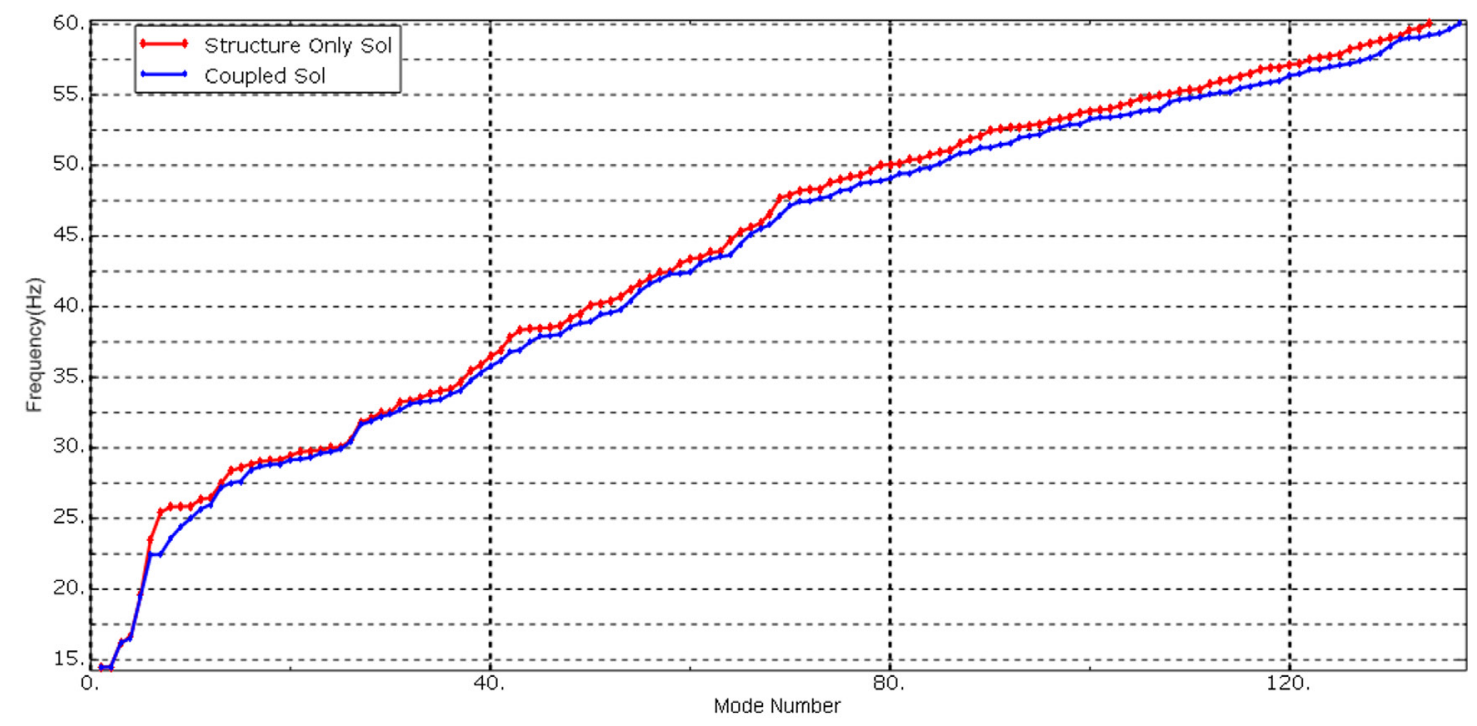

Figure 34: Natural frequencies comparison between Structural Only Sol and Coupled Solution \#2

Figure 34 shows comparison of natural frequencies of Structure only solution and Coupled solution\#2. As shown, Coupled solution\#2 has lower natural frequencies as compared to the Structure only solution due to the addition of mass of acoustic mesh. Also, there are higher number of coupled natural frequencies as compared to structure only solution. Shown below are the mode shapes at $38 \mathrm{~Hz}$ and $48 \mathrm{~Hz}$ where high acoustic sensitivity at driver's right ear was evaluated during FRF calculation.
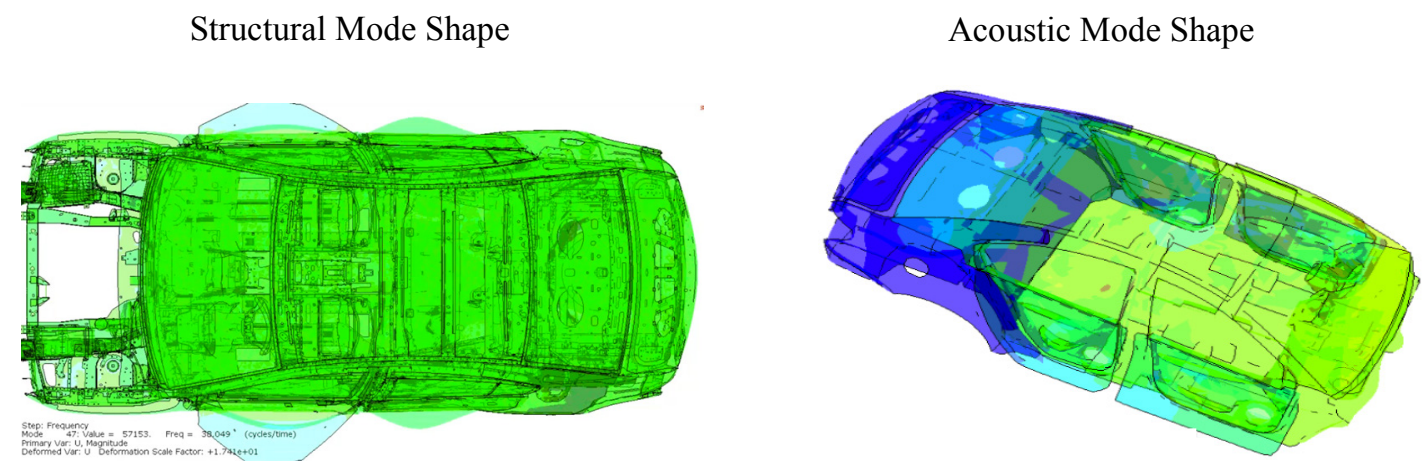

Figure 35: Coupled Mode Shapes at $38 \mathrm{~Hz}$

Figure 35 shows the Coupled Solution \#2 mode shapes at $38 \mathrm{~Hz}$. As shown, for the structural part of the mode shapes we have the front and rear doors going in-phase with each other, thus squeezing the acoustic mesh inside. On the other hand, for the acoustic mesh we have mode shape going from front to the rear of the vehicle. 

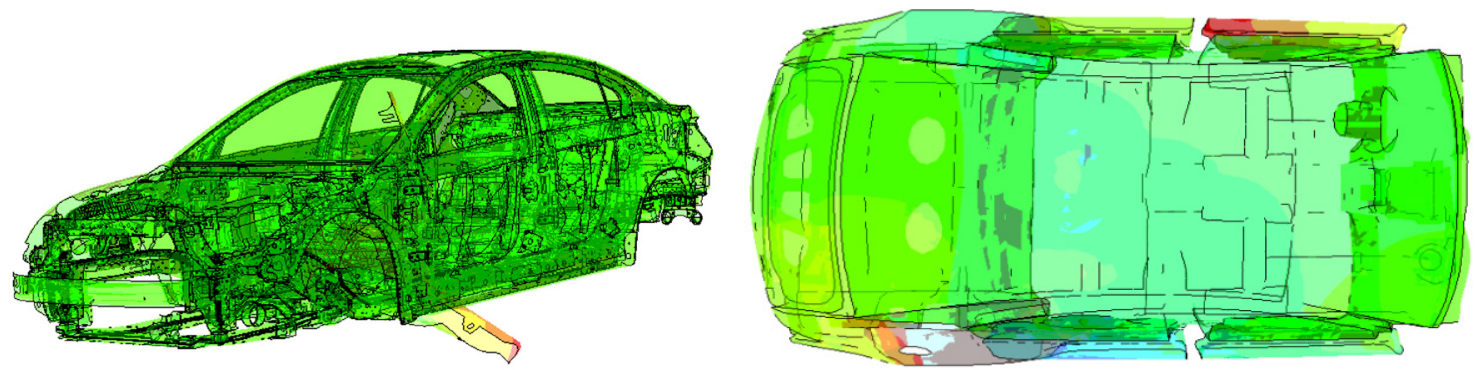

Figure 36: Coupled Mode Shapes at $48.2 \mathrm{~Hz}$

Figure 36 shows the coupled mode shapes at $48 \mathrm{~Hz}$. As shown for the structural mode shape we have a displacement on the front driver side fender. In the acoustic mode shape we have a diagonal mode shape with the front driver door and the area near the fuel pipe showing the maximum acoustic sensitivity.

\section{b. FRF Calculation for Cruze Acoustic Mesh \#2}

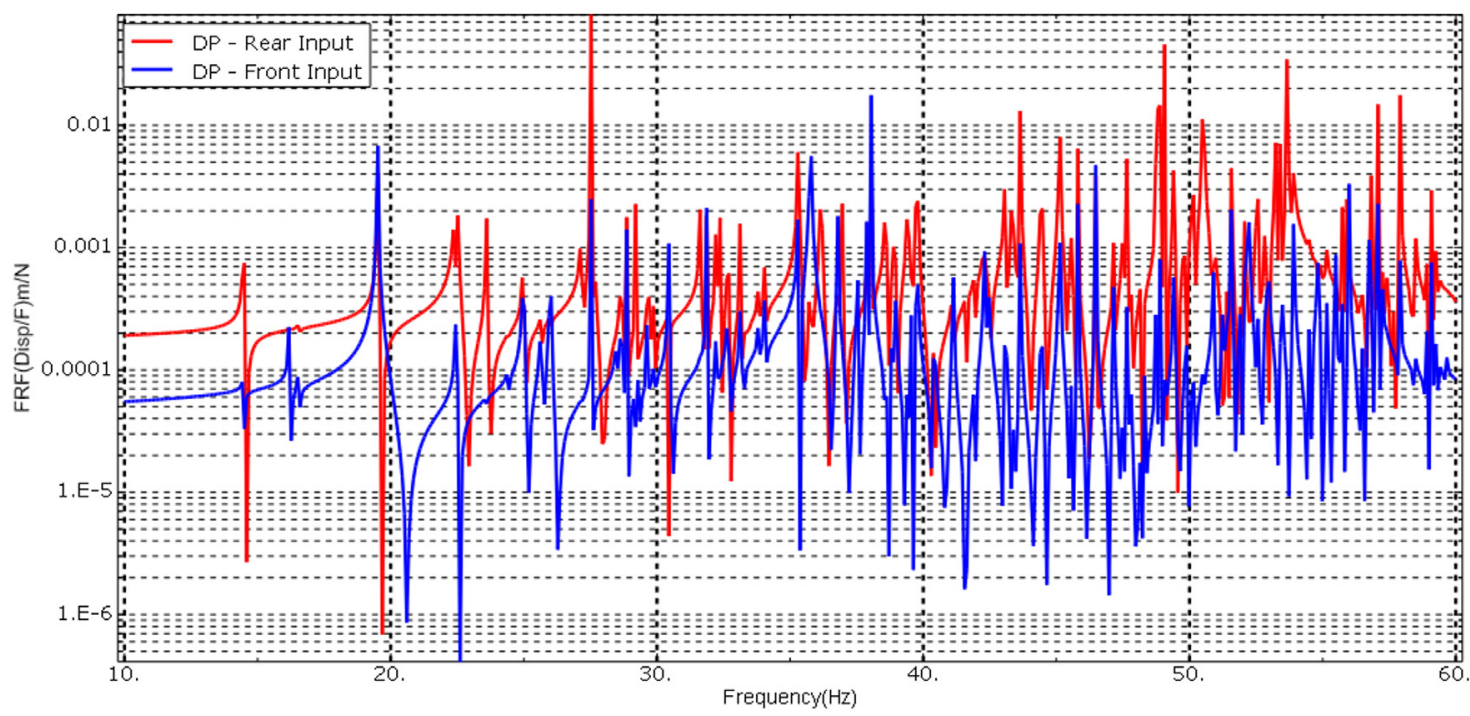

Figure 37: Drive Point FRF for Coupled Solution \#2 at front and rear input

Figure 37 shows the drive point FRF for front and rear input for Coupled Solution \#2 in the frequency range of $10 \mathrm{~Hz}$ to $60 \mathrm{~Hz}$. As shown, the response from front input is lower as compared to the rear input throughout the frequency range. 


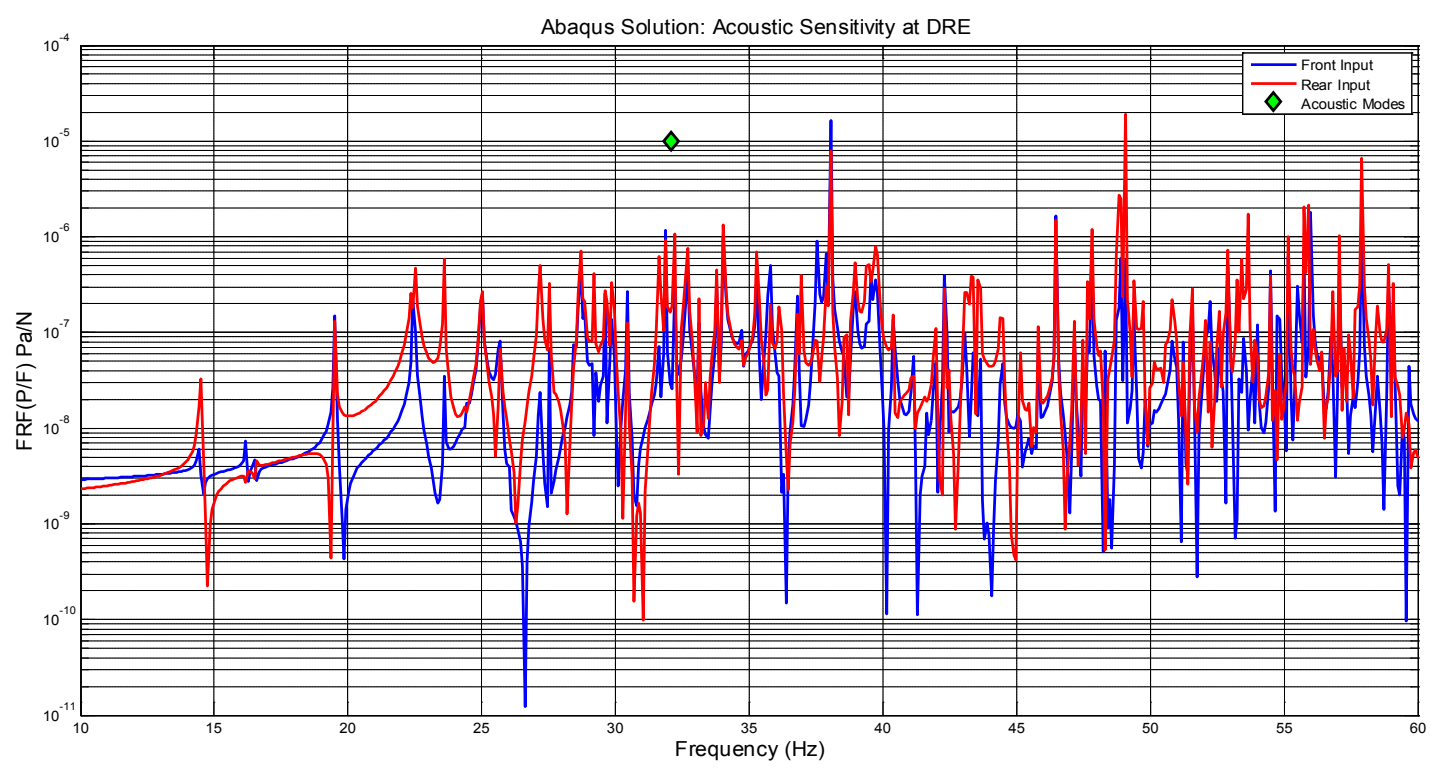

Figure 38: Acoustic sensitivity at DRE due to front and rear input

Figure 38 shows the acoustic sensitivity at DRE due to front and rear input with the green spade marker showing the first pure acoustic mode at $35 \mathrm{~Hz}$. As shown, both the front and rear inputs show a high response at $38 \mathrm{~Hz}$ at DRE.

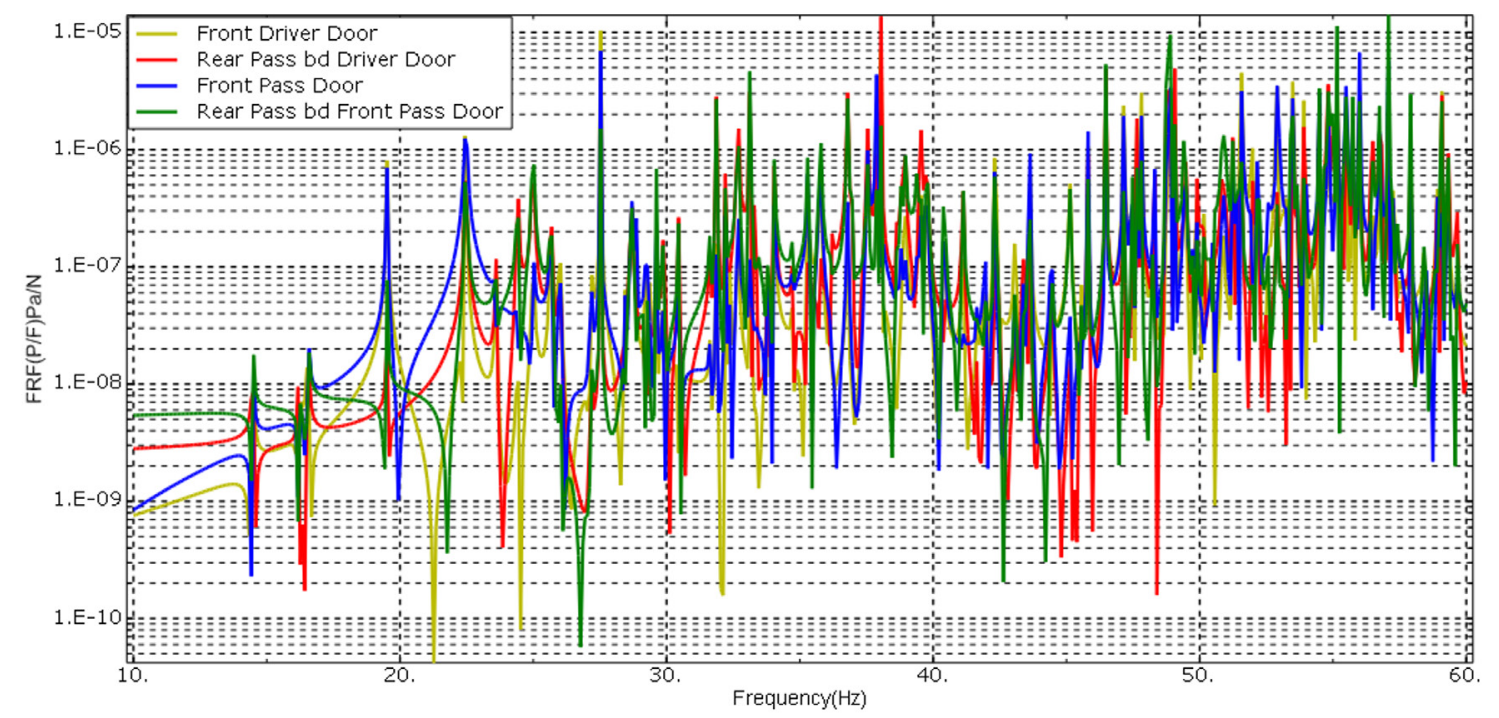

Figure 39: Acoustic sensitivities at door cavities due to front input

Figure 39 shows the acoustic sensitivities in the door cavities due to the front input. As shown, all the door cavities have a significant high acoustic pressure value at $38 \mathrm{~Hz}$, which is similar to the test results. 


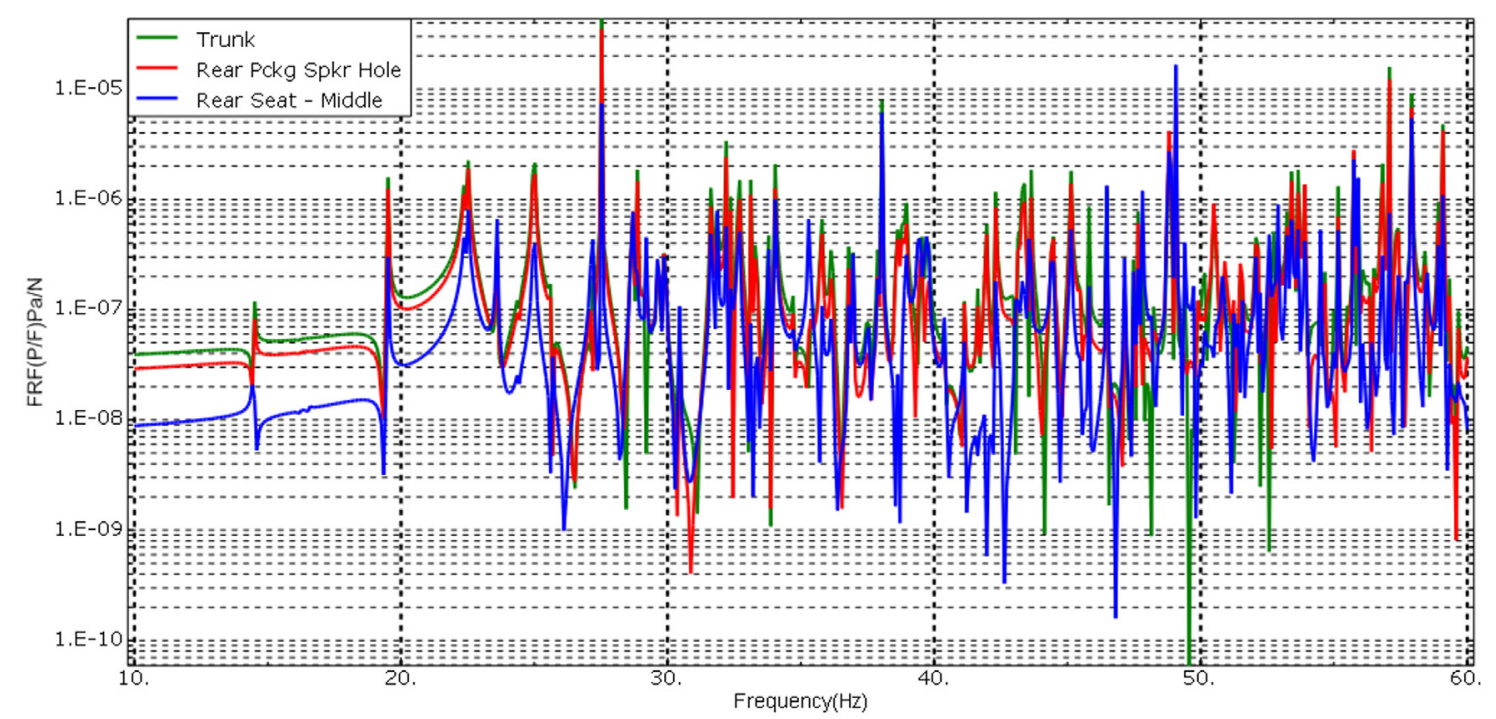

Figure 40: Acoustic Sensitivity at different points due to rear input

Figure 40 shows the acoustic sensitivity at trunk, rear seats and rear package speaker hole between $10 \mathrm{~Hz}$ to $60 \mathrm{~Hz}$ with a low acoustic sensitivity on rear seat location as the structural mesh of rear seats acts as an acoustic barrier.

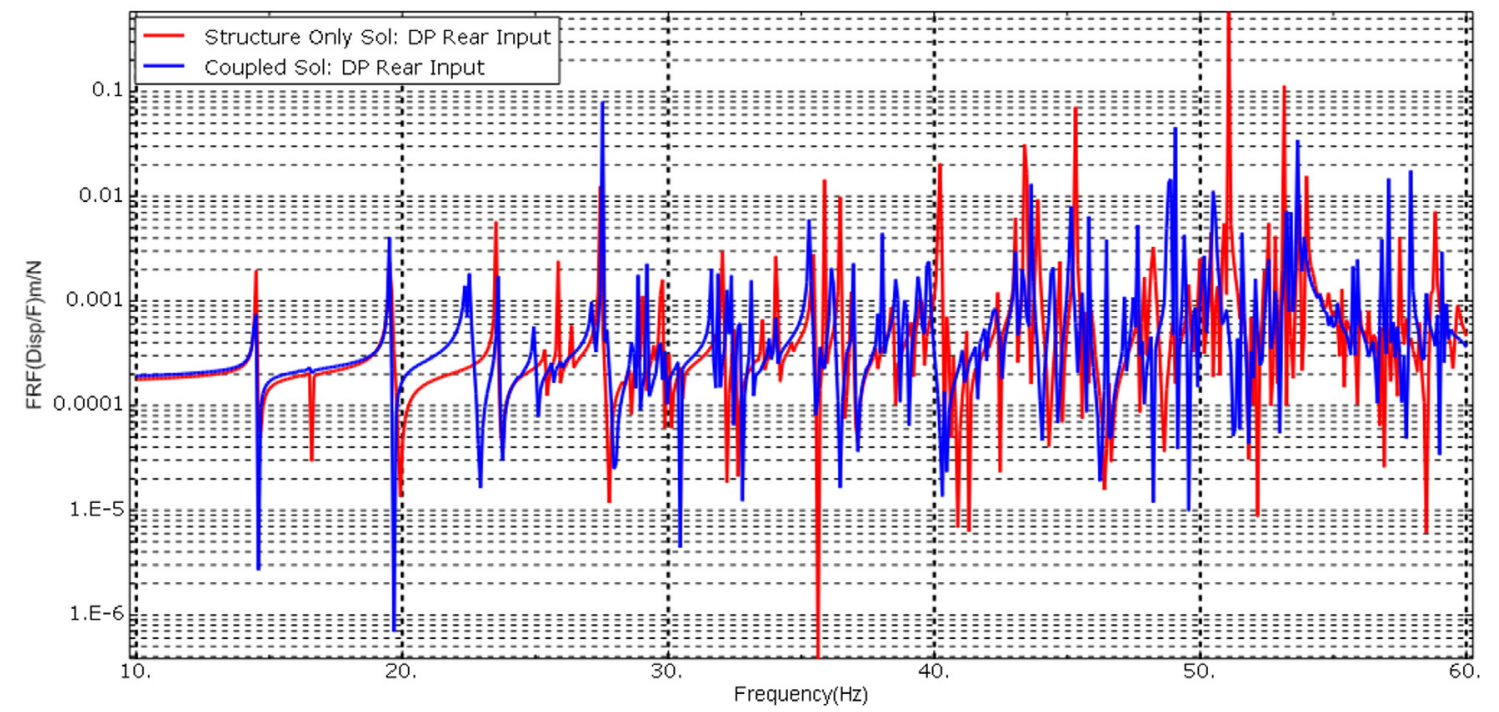

Figure 41: Drive point comparison between Structure Only Solution and Coupled Solution \#2 due to rear input

Figure 41 shows the drive point comparison between structure only solution and Coupled Solution \#2. As shown although they follow the same trend, new frequencies are introduced and also frequency shifts occur due to addition of acoustic mesh. 


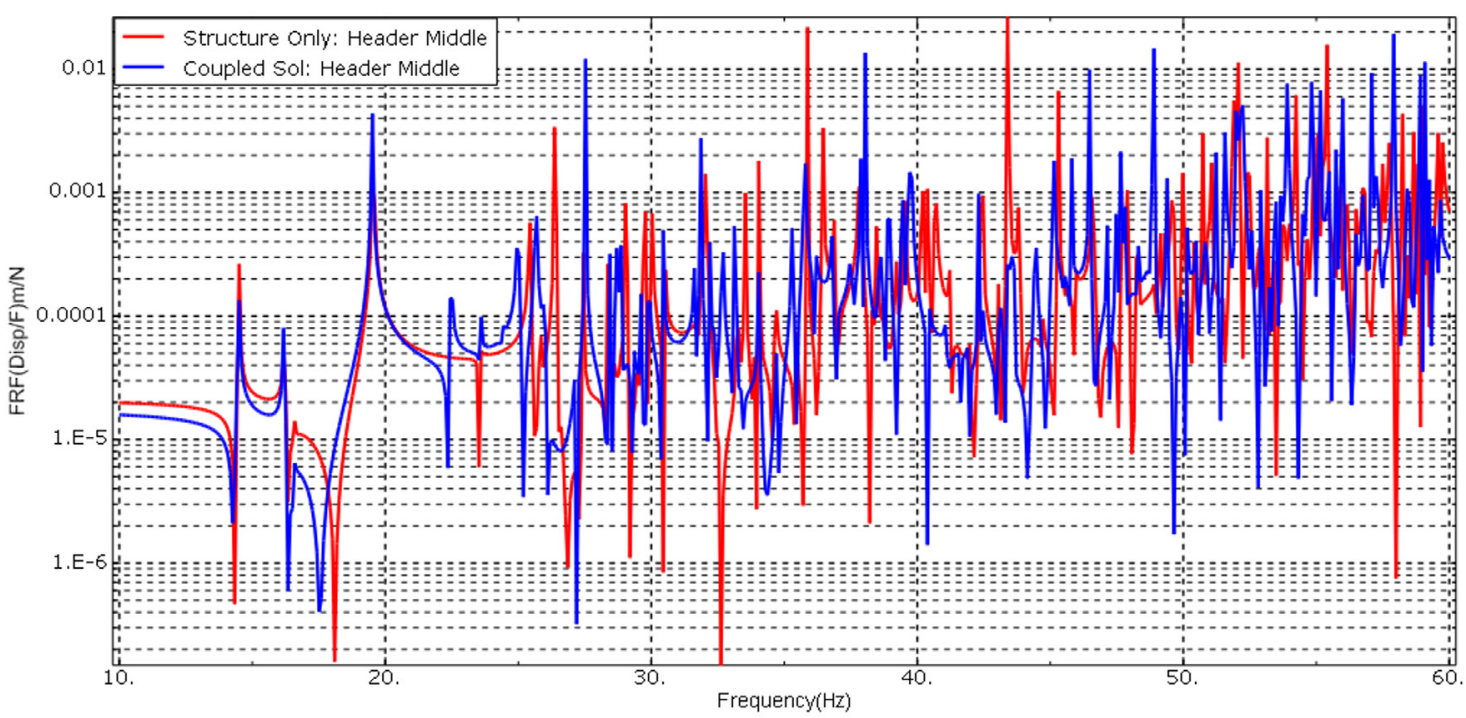

Figure 42: Comparison of Structure Only Sol and Coupled Solution \#2 for output at Header Middle due to front input

Figure 42 shows the comparison between Structural only solution and Coupled solution \#2 due to front input. As, we have high coupled solution output at $38 \mathrm{~Hz}$ and a high structural mesh output at $35 \mathrm{~Hz}$.

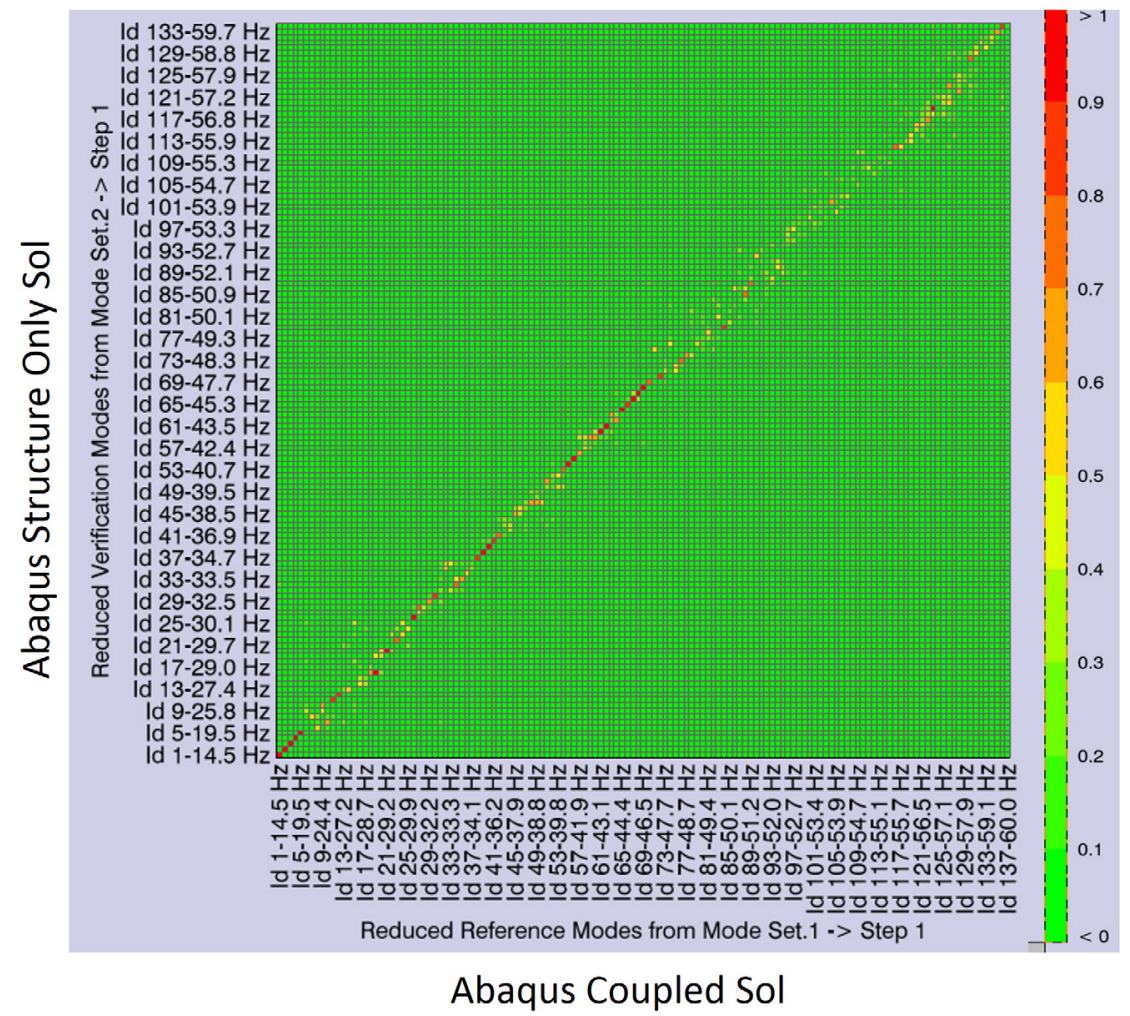

Figure 43: MAC Calculation between Coupled Solution \#2 and Structure Only Solution 
Figure 43 shows the MAC plot between Coupled solution\#2 and Structure only solution which is evaluated in LMS Virtual. Lab. As shown, MAC plot is diagonal up to $48 \mathrm{~Hz}$. At $40 \mathrm{~Hz}$, the plot is not diagonal which indicates the effect of acoustic mode shapes in the coupled mode shapes.

\subsection{Comparison of Coupled Acoustic Solution \#1 and Coupled Solution \#2}

\section{a. Testing Parameters and Results}

Testing was performed in advanced technology development center (ATDC) by Mr. Mayuresh Pathak on the Chevrolet Cruze LTZ body-in-white (BIW) structure. Full structural modal, full acoustic modal and door cavity testing was performed. Two structural inputs were provided during testing.

Key testing results were high acoustic sensitivity in the Chevrolet Cruze model at $40 \mathrm{~Hz}$. High structural output at the front header and high acoustic response in the door cavities at $40 \mathrm{~Hz}$. Based on these parameters Coupled Solution \#1 and Coupled Solution \#2 were compared with respect to the test results above mentioned above.

\section{b. Comparison between coupled solutions}

Coupled Solution\#1 and Coupled Solution\#2 are studied individually and are now compared.

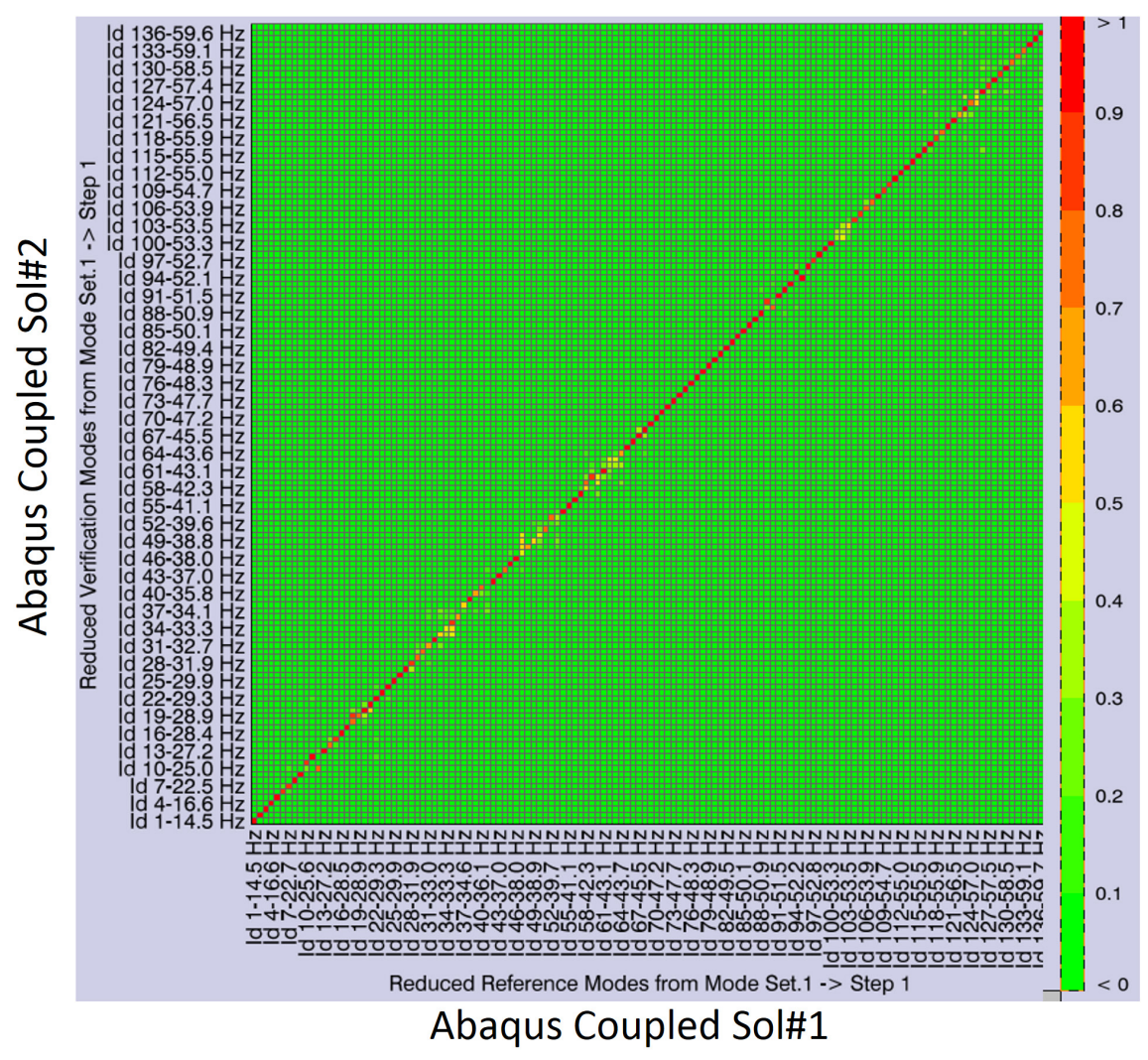

Figure 44: MAC Plot between Coupled Solution\#1 and GM Coupled Solution\#2 
Figure 44 shows MAC comparison between Coupled Solution\#1 and Coupled Solution\#2 solution. As shown, both the models have a diagonal MAC with very high MAC values, but the MAC value is 0.5 in the boom frequency range of $40 \mathrm{~Hz}$.

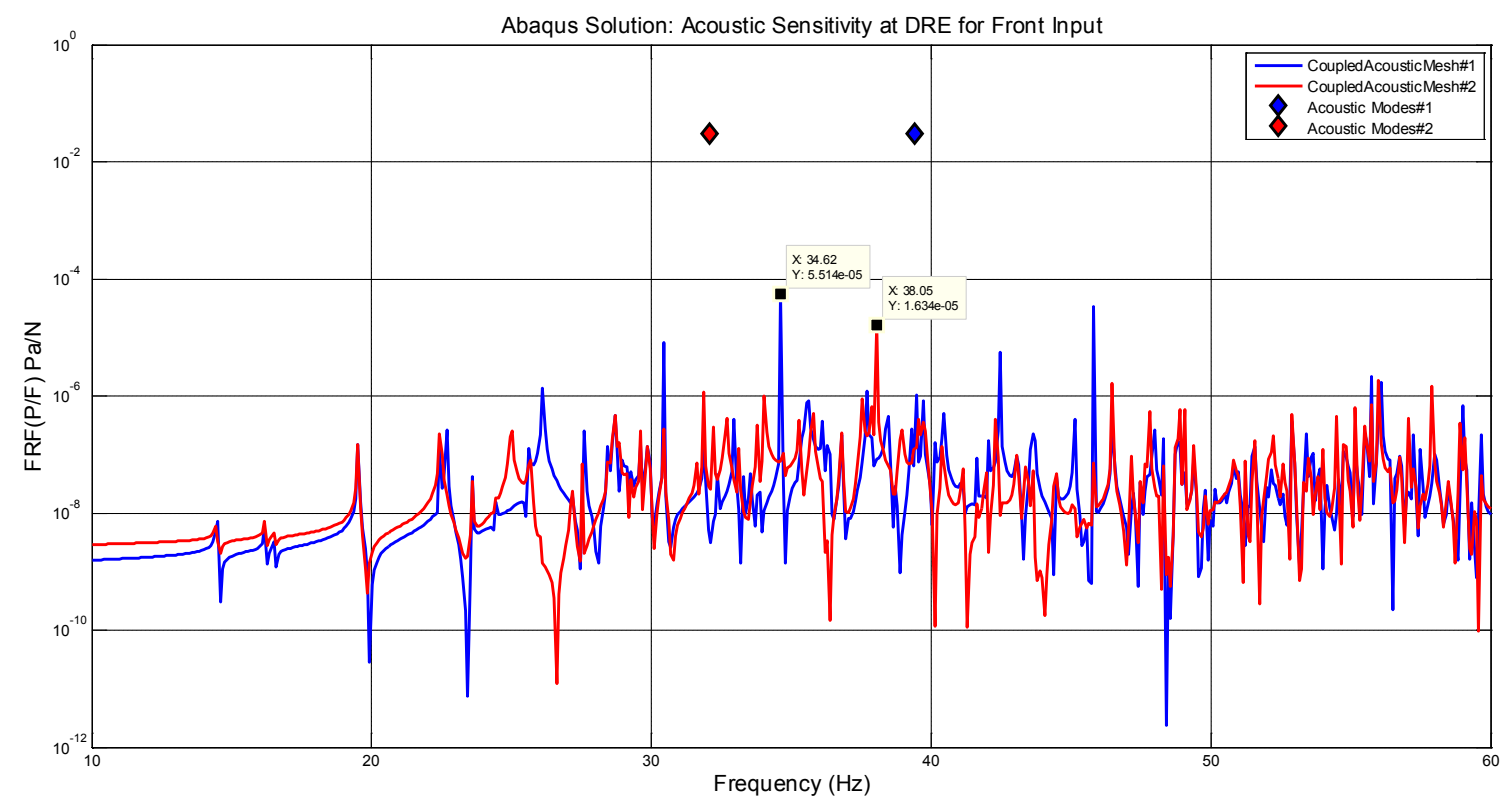

Figure 45: Comparison between Coupled Sol \#1 and Coupled Sol \#2 for acoustic sensitivity at DRE due to front input

Figure 45 shows the comparison between Coupled Solution \#1 and Coupled Solution \#2 for acoustic response at DRE. Coupled Solution \#2 with rear seats separated from the trunk in the acoustic mesh shows an acoustic boom at $38 \mathrm{~Hz}$ as compared to Coupled Solution\#1 which shows a boom at $35 \mathrm{~Hz}$. Also, the Coupled Solution \#2 acoustic mode shapes were found to be similar to the test results as compared to the Coupled Solution\#1 results.

Both the Abaqus coupled solutions and results obtained from tests were compared. Although poor modal correlation was obtained between the two results but visual comparison revealed that Abaqus results for the Coupled Solution \#2 in the boom frequency range were similar to test results with the front header showing significant response as shown in figure 42. Also high acoustic response is obtained in the door cavities in the boom frequency range as shown in figure 39. Also, for Coupled Solution \#2, acoustic boom was observed at $38 \mathrm{~Hz}$ which is closer to $40 \mathrm{~Hz}$ boom obtained during testing. Thus, Coupled Solution \#2 is better approximation of the test results as compared to Coupled Solution \#1 and Acoustic Mesh \#2 is an effective way to model the acoustic mesh of Chevrolet Cruze LTZ and should be used for further analysis. 


\section{4: Finite Element Analysis of Simple Car Models (SCM)}

It is difficult to study the effect of small components on the acoustic boom phenomenon in the huge six million degree of freedom model. Thus, a simplified box car model was designed and analyzed to study the effect of smaller components like door cavities, rear seats and rear package speaker hole on the acoustic response at driver's right ear (DRE) at $40 \mathrm{~Hz}$. During various acoustic vehicle tests it was observed that these components play a major role on the acoustic boom phenomenon.

The general procedure to design and solve all the simple car models is described in three steps as follows:

Step 1: All the simple box car models were modeled and meshed in Altair Hypermesh. Structural and acoustic meshes were modeled. The model is stored as a Nastran (.bdf) file and then converted to Abaqus (.inp) file using the 'Abaqus fromNastran' command.

Step 2: Then the models were imported into Abaqus CAE and solved for mode shapes and Eigen frequencies using the 'Linear Perturbation' procedure, 'Frequency' step and 'Lanczos' solver in Abaqus. The structural and acoustic meshes are coupled using 'tie constraints'. Structural inputs were provided and frequency response at DRE is evaluated using 'Modal Steady State Dynamics' step.

Step 3: Post processing is performed in Altair Hyperview. Visual comparison is performed with the test results and comparison with different SCM's is performed to study the effect of different components.

\subsection{Simple Car Model \#1 (SCM\#1)}

Initially, a simple car model was modeled to mimic the overall structure of the GM Cruze car model. The dimensions of the car and components like hood and trunk of the car are similar to Chevrolet Cruze dimensions but the structure is a simple box. The simple car model\#1 attributes are described below.

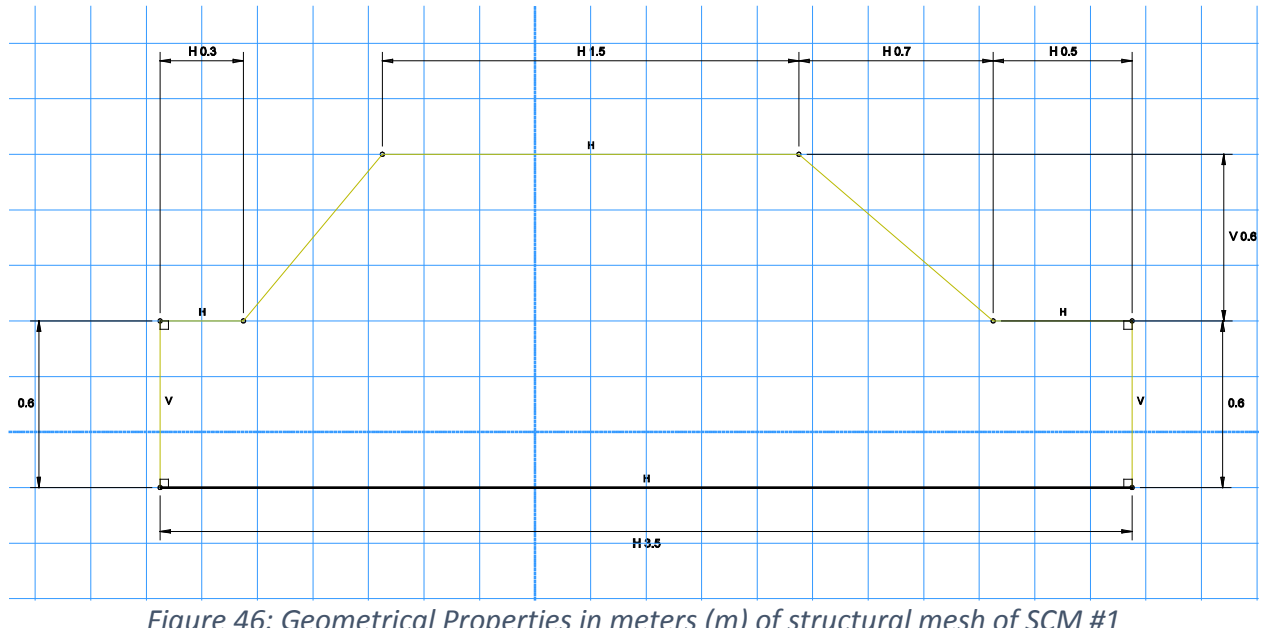

Figure 46: Geometrical Properties in meters $(m)$ of structural mesh of SCM \#1 
As shown in figure 46, SCM\#1 has the overall dimensions of the Chevrolet Cruze LTZ model with a width of $1 \mathrm{~m}$.

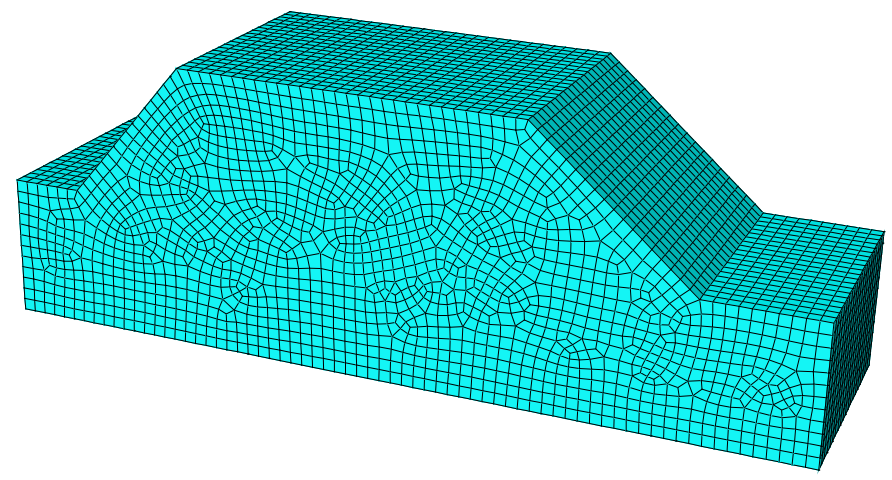

Figure 47: Structural Mesh of Simple Car Model\#1

Figure 47 shows the Simple Car Model \#1 mesh model. As shown, the model has the trunk and hood of the car as simple rectangular box structure. It is made entirely of shell elements and no beams are present in the model. The model attributes are described below.

Table 3: Structural Mesh attributes of Simple Car Model \#1

\begin{tabular}{|l|l|}
\hline Element Type & CQUAD4 (Shells) \\
\hline Material Properties & $\begin{array}{l}\text { Young's Modulus: } 200 \mathrm{GPa} \\
\text { Poisson Ratio: } 0.3 \\
\text { Density : 7800 Kg/m }\end{array}$ \\
\hline Number of Nodes & 6268 \\
\hline Number of Elements & 6266 \\
\hline Model Mass & $306.988 \mathrm{Kg}$ \\
\hline
\end{tabular}

Table 4: Acoustic Mesh attributes of Simple Car Model \#1

\begin{tabular}{|l|l|}
\hline Element Type & Tetrahedral \\
\hline Number of Nodes & 36295 \\
\hline Number of Elements & 199982 \\
\hline
\end{tabular}


Table 3 and Table 4 show the structural and acoustic mesh attributes for SCM \#1 respectively. The simple car model\#1 had low structural stiffness with the given material properties and Eigen solution obtained did not represent the Chevrolet Cruze LTZ finite element model results. Thus, the Simple Car Model\#1 was modified by adding beam elements to make the structure stiffer and Simple Car Model \#2 was created.

\subsection{Simple Car Model \#2 (SCM\#2)}

Simple car model \#2 is modeled to study the effect of door cavities on the acoustic boom phenomenon. Door cavities have a hollow volume between the inner and outer door panels. We have modeled two variants of SCM \#2, simple car model \#2A is modeled without the door cavity. On the other hand, simple car model \#2B is modeled as a car with the door cavities blocked off, so the volume of the inside acoustic cavity is reduced. Finally, a MAC comparison graph is plotted between the pure acoustic and coupled acoustic modes. This graph shows the mapping of pure acoustic modes onto coupled system vibro-acoustic modes.

\subsubsection{Simple Car Model \#2A (SCM \#2A)}

Simple Car Model \#2A Structural Mesh: The structural mesh of the Simple car model\#2 is modified by adding beam and spring elements to the shell elements to the simple car model\#1.

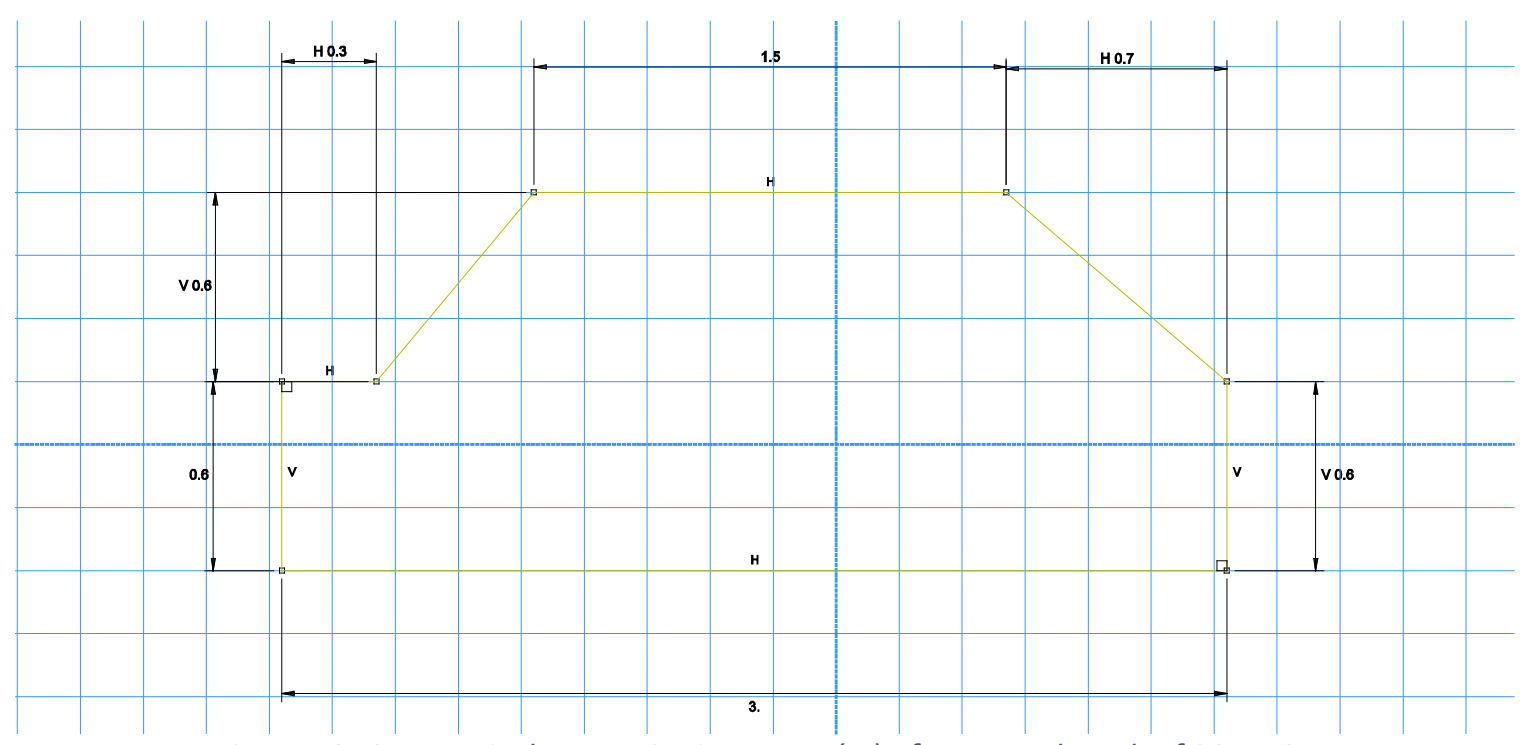

Figure 48: Geometrical Properties in meters $(m)$ of structural mesh of SCM \#2A

Figure 48 shows the geometrical properties of SCM\#2A. As shown all the major dimensions of SCM\#2A are same as SCM\#1, but the hood of the car is removed, as it does not influence the acoustic boom phenomenon. 


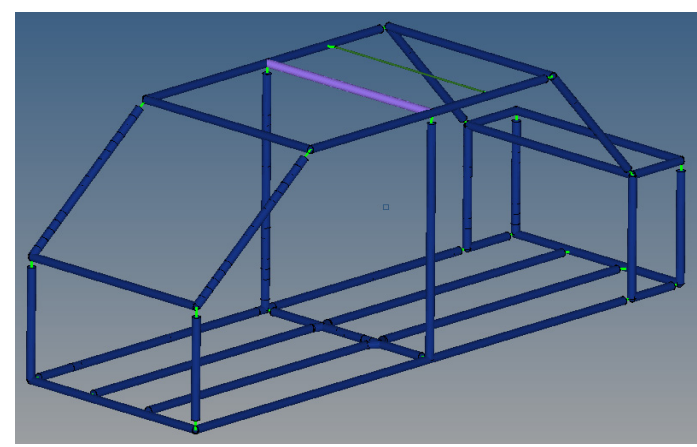

Figure 49: SCM\#2A beam structure

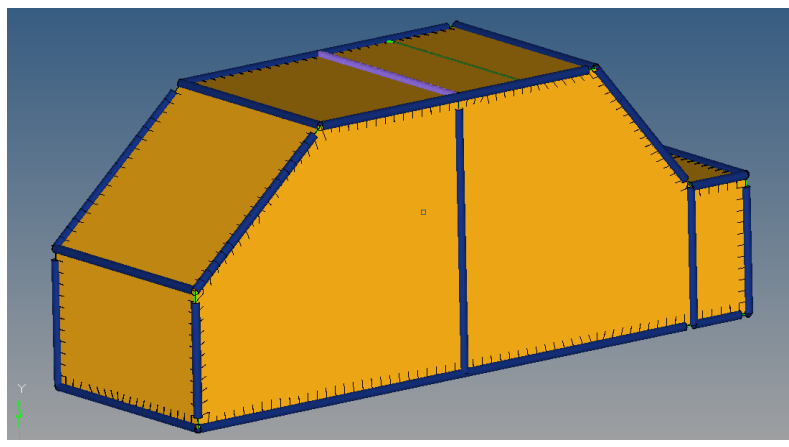

Figure 50: SCM\#2A with shell and beam elements

Figure 49 shows the complete simple car model structural mesh with beam and shell elements and figure 50 shows the beam structure for structural mesh of SCM \#2A. As shown, the structural mesh is made of beam elements that stiffen the structure. SCM \#2A has natural frequencies and modes shapes similar to the actual Chevrolet Cruze LTZ finite element structural mesh. The beam elements are joined to the shell elements using equivalency method in Altair Hypermesh. Also, the beams have spring elements between them to get the desired stiffness. Also, the doors made of shell elements are attached to the main body of the car using spring elements.

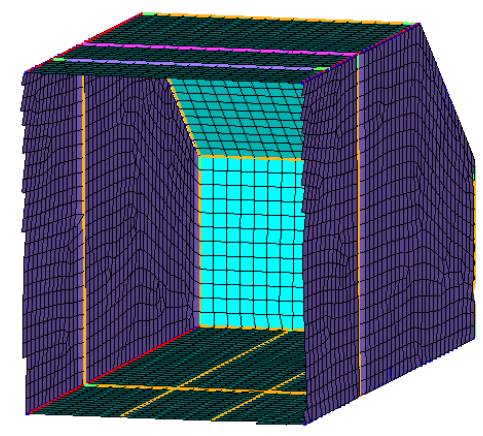

Figure 51: Cut Section of SCM\#2A Structural mesh

Figure 51 shows the SCM \#2 structural mesh cut view. As shown the inner door panels are not modeled in the structural mesh. Table 5 shows the model attributes of the simple car model \#2 structural mesh.

Table 5: Structural Mesh attributes of Simple Car Model\#2

\begin{tabular}{|l|l|}
\hline Element Size & $50 \mathrm{~mm}$ \\
\hline Nodes & 5868 \\
\hline Elements & 6539 \\
\hline
\end{tabular}




\begin{tabular}{|l|l|}
\hline Mass & $422.24 \mathrm{Kg}$ \\
\hline Door Thickness & $5 \mathrm{~mm}$ \\
\hline Thickness-Rest & $2.5 \mathrm{~mm}$ \\
\hline Material Properties & $\begin{array}{l}\text { Young's Modulus: } 200 \mathrm{GPa} \\
\text { Poisson Ratio: 0.3 } \\
\text { Density: } 7800 \mathrm{Kg} / \mathrm{m}^{3}\end{array}$ \\
\hline Beams & $\begin{array}{l}\text { Density: } 7800 \mathrm{Kg} / \mathrm{mm}^{3} \\
\text { Young's Modulus: } 200 \mathrm{GPa} \\
\text { Shear Modulus: } 76 \mathrm{GPa}\end{array}$ \\
\hline Springs & $\begin{array}{l}\text { 6 degrees of freedom } \\
\text { Translational Stiffness: } 10 \mathrm{E} 9 \mathrm{~N} / \mathrm{mm}^{2} \\
\text { Rotational Stiffness: } 7000 \mathrm{~N} / \mathrm{mm}^{2}\end{array}$ \\
\hline
\end{tabular}

As shown in table 5, the structural mesh has different thicknesses for doors and rest of the structural mesh. Door seals are modeled using spring elements. The SCM \#2A structural mesh is now solved using Abaqus solver to evaluate the natural frequencies and mode shapes.

Simple Car Model\#2A Acoustic Mesh: Simple car model\#2A acoustic mesh has the same geometrical dimensions as the SCM \#2A structural mesh. Thus, it represents the condition with no door cavities and so there is a large seamless acoustic cavity.

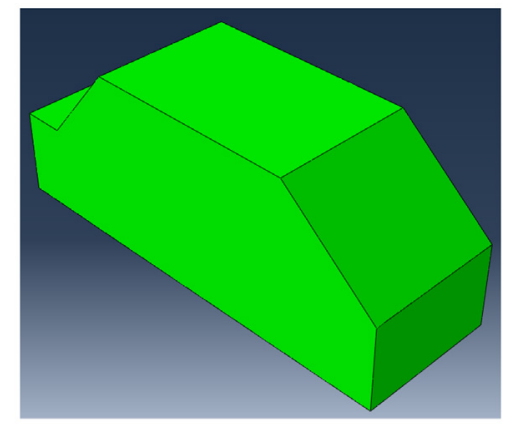

Figure 52: SCM \#2A Acoustic Mesh

Figure 52 shows the Simple Car Model \#2A acoustic mesh. As shown, it is a large acoustic cavity modeled in Hypermesh using linear tetrahedral (C3D4) elements. Table 6 shows the mesh attributes if Acoustic Mesh \#2A. 
Table 6: Acoustic Mesh attributes of Simple Car Model\#2A

\begin{tabular}{|l|l|}
\hline Nodes & 15065 \\
\hline Elements & 78618 \\
\hline Element Type & Linear Tetrahedral (C3D4) \\
\hline Mass & $3.75 \mathrm{Kg}$ \\
\hline
\end{tabular}

The SCM \#2A acoustic mesh is solved in Abaqus for natural frequencies and mode shapes. Thus, pure acoustic mode shapes and natural frequencies are obtained. Also, SCM \#2A structural and acoustic meshes are coupled together using tie constraints with the 'default' tolerance value in Abaqus and natural frequencies and mode shapes of the coupled system are evaluated and the response at driver's right ear (DRE) is evaluated for the input on SCM \#2A structural mesh. Acoustic modes of the coupled acoustic-structural mesh and pure acoustic modes are evaluated and compared using MAC. The subsequent MAC plots are generated after exporting the acoustic pressure values for the pure acoustic and coupled acoustic modes over a specific frequency range from Abaqus using python scripting. These values were then imported into Matlab and plotted.

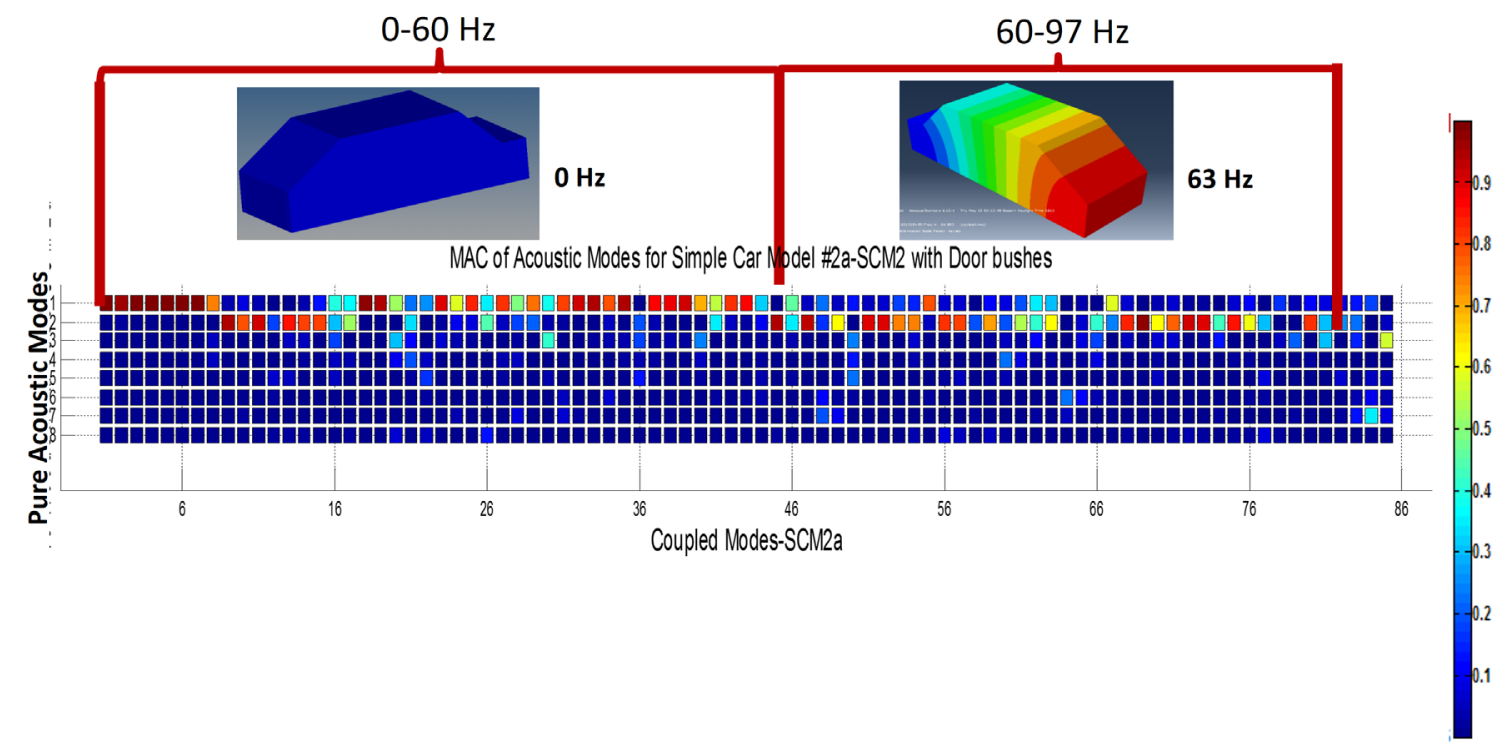

Figure 53: MAC comparison between SCM \#2A pure acoustic and SCM \#2A coupled acoustic mode shapes

Figure 53 shows MAC plot between pure acoustic mode shapes on the vertical axis and coupled acoustic mode shapes on the horizontal axis for SCM\#2A up to $100 \mathrm{~Hz}$. The first pure acoustic mode shape participates in the coupled modes from $0 \mathrm{~Hz}$ to $60 \mathrm{~Hz}$ which is the boom frequency range and second pure acoustic mode shape participates in the coupled 
mode shapes from $60 \mathrm{~Hz}$ to $97 \mathrm{~Hz}$. Thus, as shown one pure acoustic mode participates over a band of coupled vibro-acoustic modes.

\subsubsection{Simple Car Model\#2B (SCM \#2B)}

Simple Car Model \#2B Structural Mesh: SCM \#2B is modeled to study the effect after blocking-off the door cavities from the main cavity. Thus, SCM \#2B structural mesh is modeled differently with no door cavities, but all the major dimensions remain the same as $\mathrm{SCM} \# 2 \mathrm{~A}$. The length of removed door cavities is $2.7 \mathrm{~m}$, width of $0.063 \mathrm{~m}$ and a height of $0.6 \mathrm{~m}$.

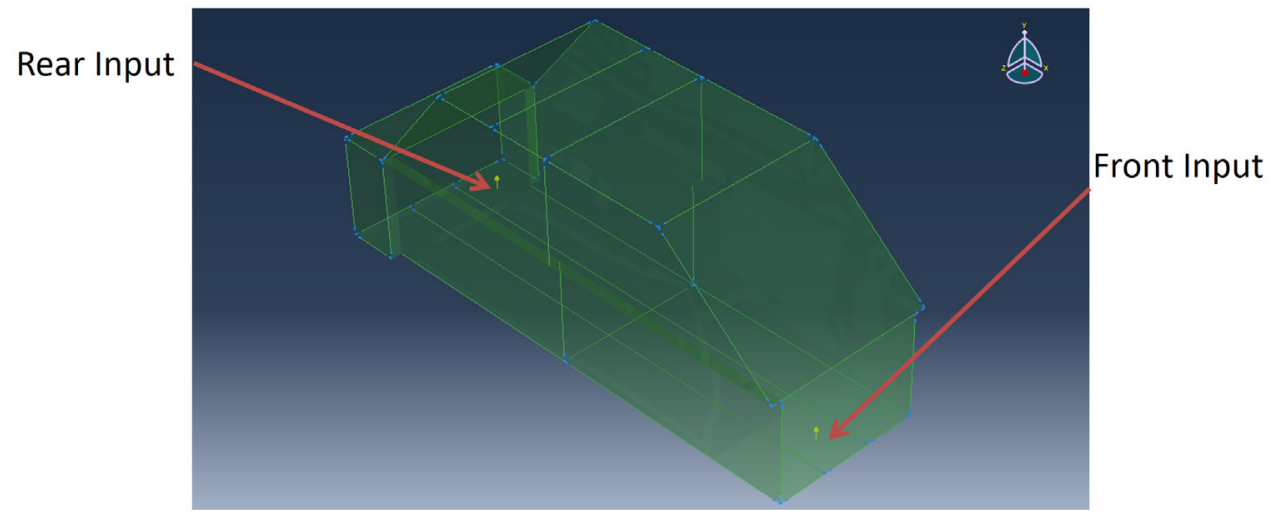

Figure 54: Structural input points for SCM \#2B

Figure 54 shows the structural mesh of SCM \#2B. As shown, the structural mesh is different with door cavities removed from main body of simple car model, but all the beam and shell material properties remain the same. There are two input points and the output is calculated at the driver's right ear (DRE) for the SCM \#2B coupled solution. As shown, in $\mathrm{SCM} \# 2 \mathrm{~B}$ the front input is at the center as opposed to a corner for SCM \#2A.

Simple Car Model \#2B Acoustic Mesh: A Simple Car Model \#2B acoustic mesh is modeled as the car model without door cavities with the geometrical dimensions as SCM\#2B structural mesh.

Table 7: Acoustic Mesh attributes of Simple Car Model\#2B

\begin{tabular}{|l|l|}
\hline Nodes & 10110 \\
\hline Elements & 46208 \\
\hline Element Type & Linear tetrahedral(C3D4) \\
\hline Mass & $3.50 \mathrm{Kg}$ \\
\hline
\end{tabular}




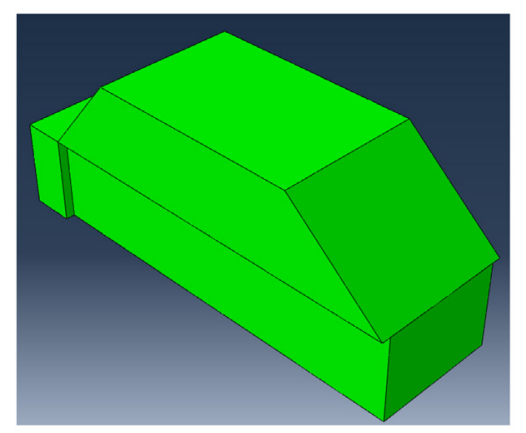

Figure 55: SCM \#2B Acoustic Mesh

Figure 69 shows SCM \#2B acoustic mesh. As shown, volume of acoustic cavity is smaller due to the removal of door cavities from the acoustic mesh. This mimics the effect of the MIMO full acoustic test on the Chevrolet Cruze LTZ car with the door cavities blocked off from the main acoustic cavity using half inch OSB panels. So, the main cavity is acoustically smaller without the door cavities. SCM\#2B acoustic mesh is solved in Abaqus to calculate the pure acoustic mode shapes and natural frequencies. The SCM \#2B acoustic and structural meshes are coupled together using tie constraints in Abaqus and solved for natural frequencies and mode shapes.

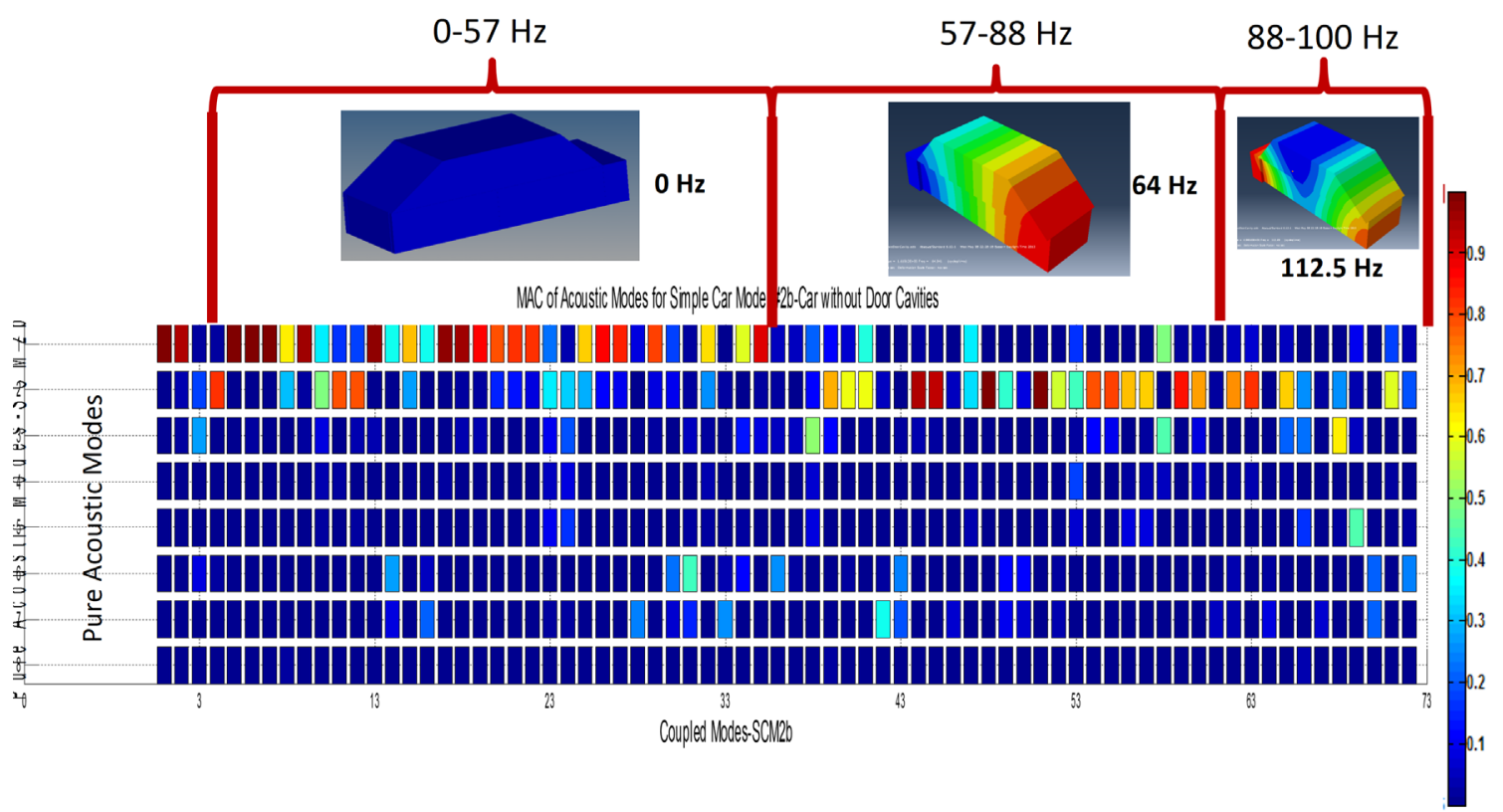

Figure 56: MAC comparison between SCM \#2B pure acoustic and SCM\#2B coupled acoustic mode shapes

Figure 56 shows the MAC plot between SCM\#2B pure acoustic and coupled acoustic mode shapes up to $100 \mathrm{~Hz}$. As shown, the pure acoustic mode shapes are along the vertical axis and coupled mode shapes are along the horizontal axis. The first pure acoustic natural frequency at $0 \mathrm{~Hz}$ participates in the coupled mode shapes from $0-57 \mathrm{~Hz}$ and second pure 
acoustic mode shape which is the front-back longitudinal mode at $64 \mathrm{~Hz}$ participates in the coupled vibro-acoustic mode shapes from 57 to $88 \mathrm{~Hz}$.

\subsection{Simple Car Model \#3 (SCM\#3)}

Simple car model\#3 was designed to study the effect of speaker openings on the door cavities. Speaker openings act as an access for air from the rest of cavity to interact with the air inside the door cavity. In simple car model \#3, the door cavities are modeled with the ratio of volume of rest of cavity to volume of door cavity is equal to the Chevrolet Cruze LTZ model.

\subsubsection{Simple Car Model \#3A (SCM\#3A)}

Simple Car Model \#3A Structural Mesh: Simple Car Model \#3A structural mesh is the same mesh used for SCM \#2A and the changes are only made in the acoustic mesh.

Simple Car Model \#3A Acoustic Mesh: A simple car model \#3A acoustic mesh is modeled in Hypermesh and then translated and solved in Abaqus. In this model door cavities are modeled with one access that connects the main cavity to the acoustic door cavity.

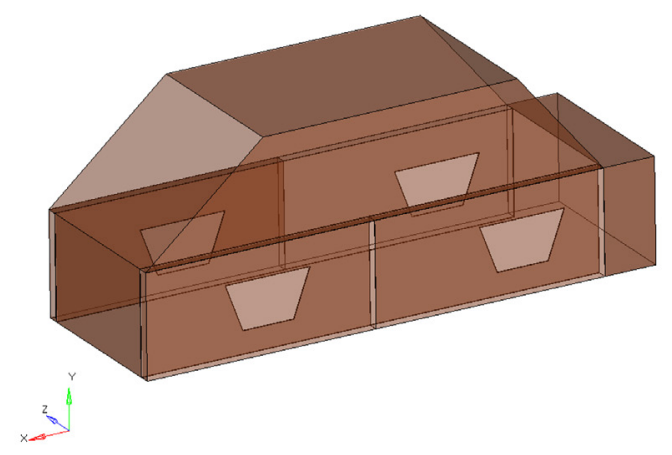

Figure 57: SCM \#3A Acoustic Mesh with one access for each door

As, shown in figure 57, the main acoustic cavity is joined to the door cavities using trapezoidal accesses with one access for each door. 


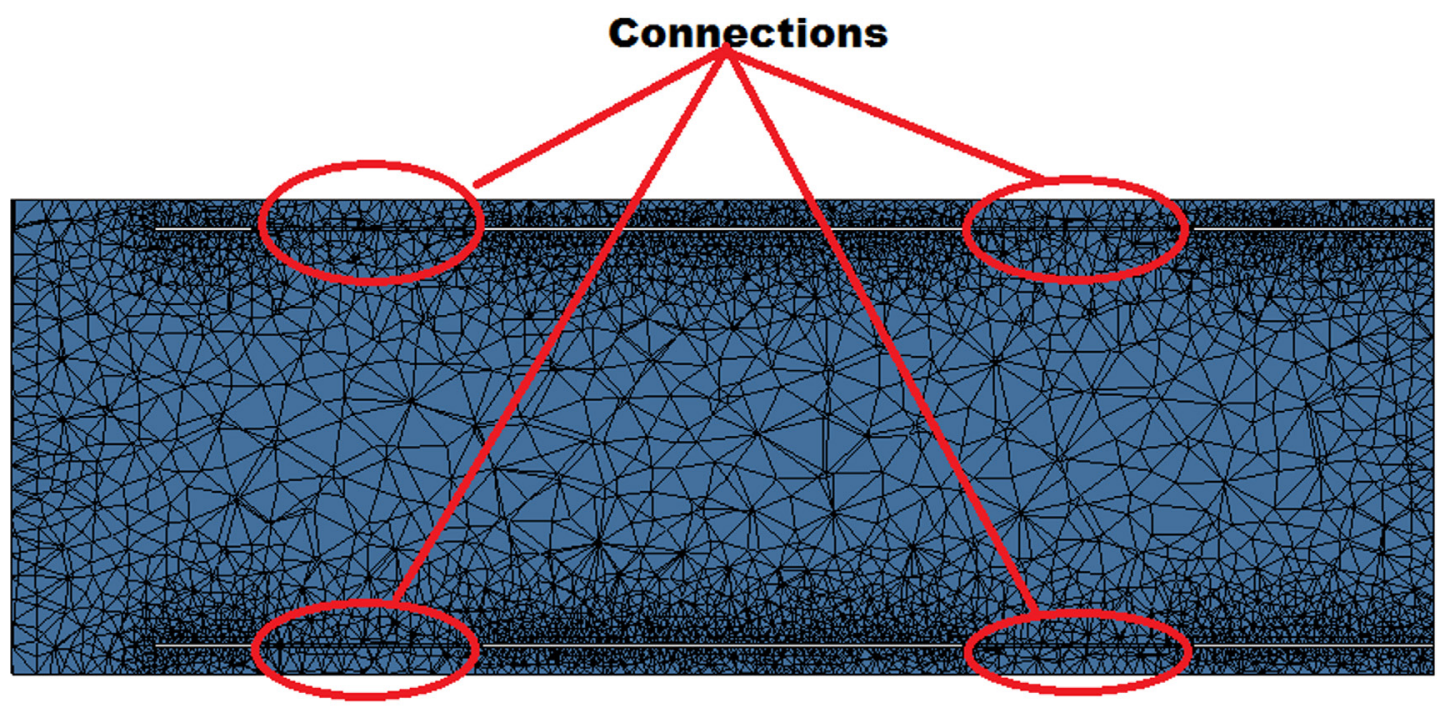

Figure 58: Connections between door cavities and main acoustic cavity for SCM \#3A

Figure 58 shows the connections between door cavities and the rest of acoustic cavity. As shown, the door cavities and the main cavities are separated from each other by a small distance and are only connected through the opening or access.

Table 8: SCM \#3A Acoustic Mesh Properties

\begin{tabular}{|l|l|}
\hline Element Size & $60 \mathrm{~mm}$ \\
\hline Nodes & 213768 \\
\hline Elements & $993181(\mathrm{C} 3 \mathrm{D} 4)$ \\
\hline Mass & $3.73 \mathrm{Kg}$ \\
\hline
\end{tabular}

Table 8 shows the SCM\#3A acoustic mesh properties. Applying the same procedure as for SCM \#2A and SCM \#2B, the structural and acoustic meshes of SCM \#3A are coupled and natural frequencies and mode shapes are evaluated. Now, MAC is evaluated between the pure acoustic modes and coupled acoustic modes to see how acoustic modes affect the coupled vibro-acoustic modes in the frequency range of 0 to $100 \mathrm{~Hz}$. 


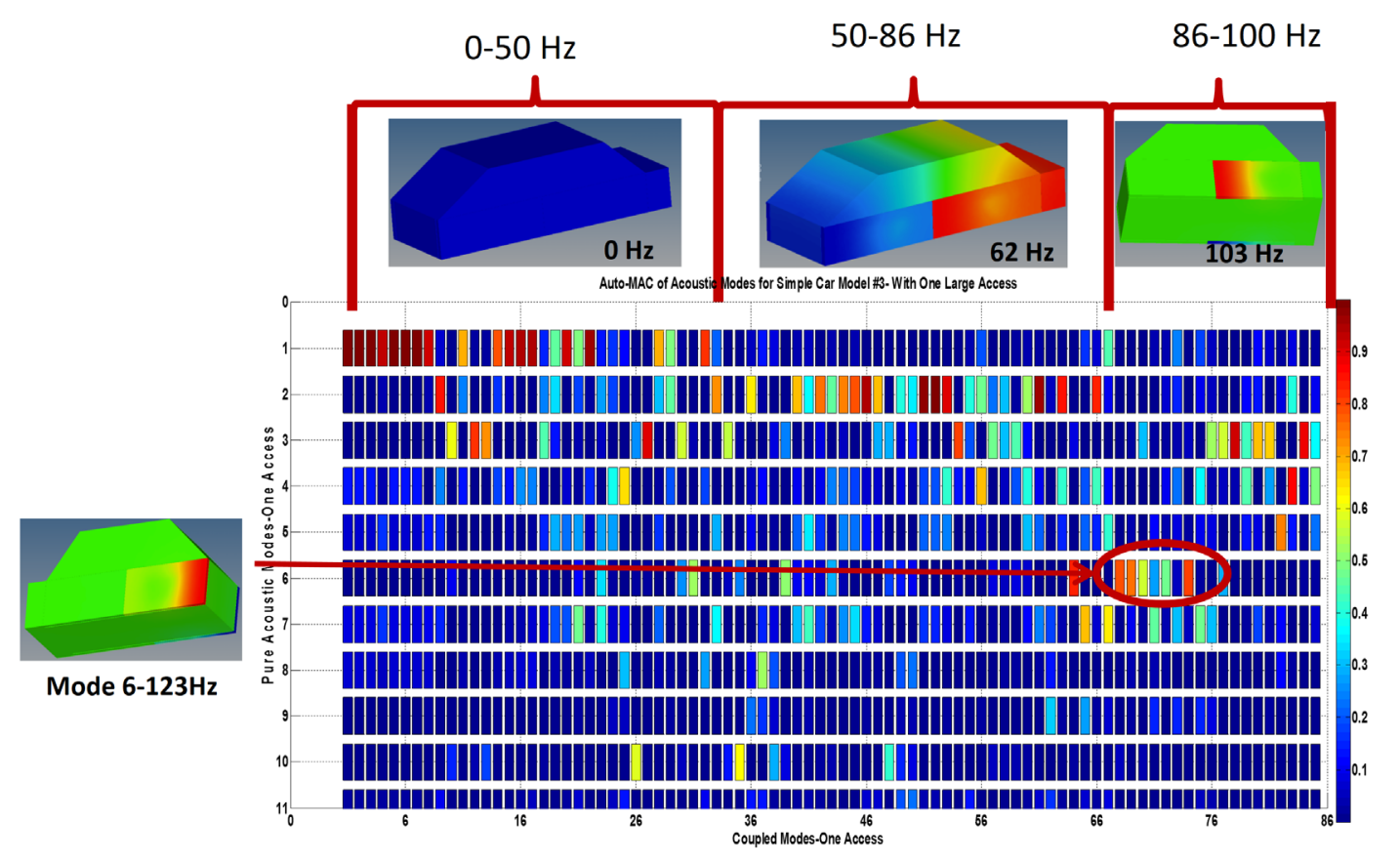

Figure 59: MAC comparison between SCM \#3A pure acoustic modes and SCM \#3Acoupled mode shapes

As shown, horizontal axis at the top represents pure acoustic modes for SCM\#3A and their frequency range of influence on the coupled mode shapes. The coupled mode shapes are along the horizontal axis. As shown, from 0 to $50 \mathrm{~Hz}$ we have participation of first mode at $0 \mathrm{~Hz}$ and from $50-86 \mathrm{~Hz}$, the second acoustic mode at $62 \mathrm{~Hz}$ has participation and from $86-100 \mathrm{~Hz}$ the third pure acoustic mode at $103 \mathrm{~Hz}$ has the maximum participation. Also, in the $86-100 \mathrm{~Hz}$ frequency range we have the participation of mode 6 which is at $123 \mathrm{~Hz}$. Thus, high frequency pure acoustic modes also participate in a band of lower frequency coupled vibro-acoustic coupled modes.

\subsubsection{Simple Car Model \#3B (SCM\#3B)}

Simple Car Model \#3B Structural Mesh: The same structural mesh as for Simple Car Model \#2A was used in SCM \#3B.

Simple Car Model \#3B Acoustic Mesh: Simple Car Model \#3B is modeled with door cavities that are connected to the main cavity by two access for each door. 


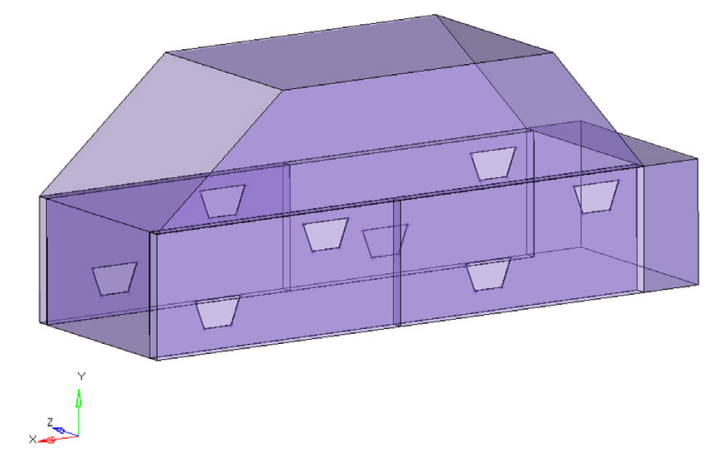

Figure 60: Simple Car Model \#3B acoustic mesh with two access for each door cavities

Figure 60 shows the simple car model \#3B acoustic mesh. As shown there are two door accesses for each door. The total area of the two openings is equal the area of opening one access in SCM \#3A. The connections between the door cavities and rest of the acoustic cavity is the same as explained in Figure 12.

Table 9: SCM \#3A Acoustic Mesh attributes

\begin{tabular}{|c|c|}
\hline Element Size & $60 \mathrm{~mm}$ \\
\hline Nodes & 153878 \\
\hline Elements & $703691(\mathrm{C} 3 \mathrm{D} 4)$ \\
\hline Mass & $3.73 \mathrm{Kg}$ \\
\hline
\end{tabular}

Table 9 gives the acoustic mode attributes. As shown, it contains 703691 C3D4 elements with an element size of $60 \mathrm{~mm}$. Similar to SCM\#3A, the model is solved in Abaqus and MAC is evaluated. 


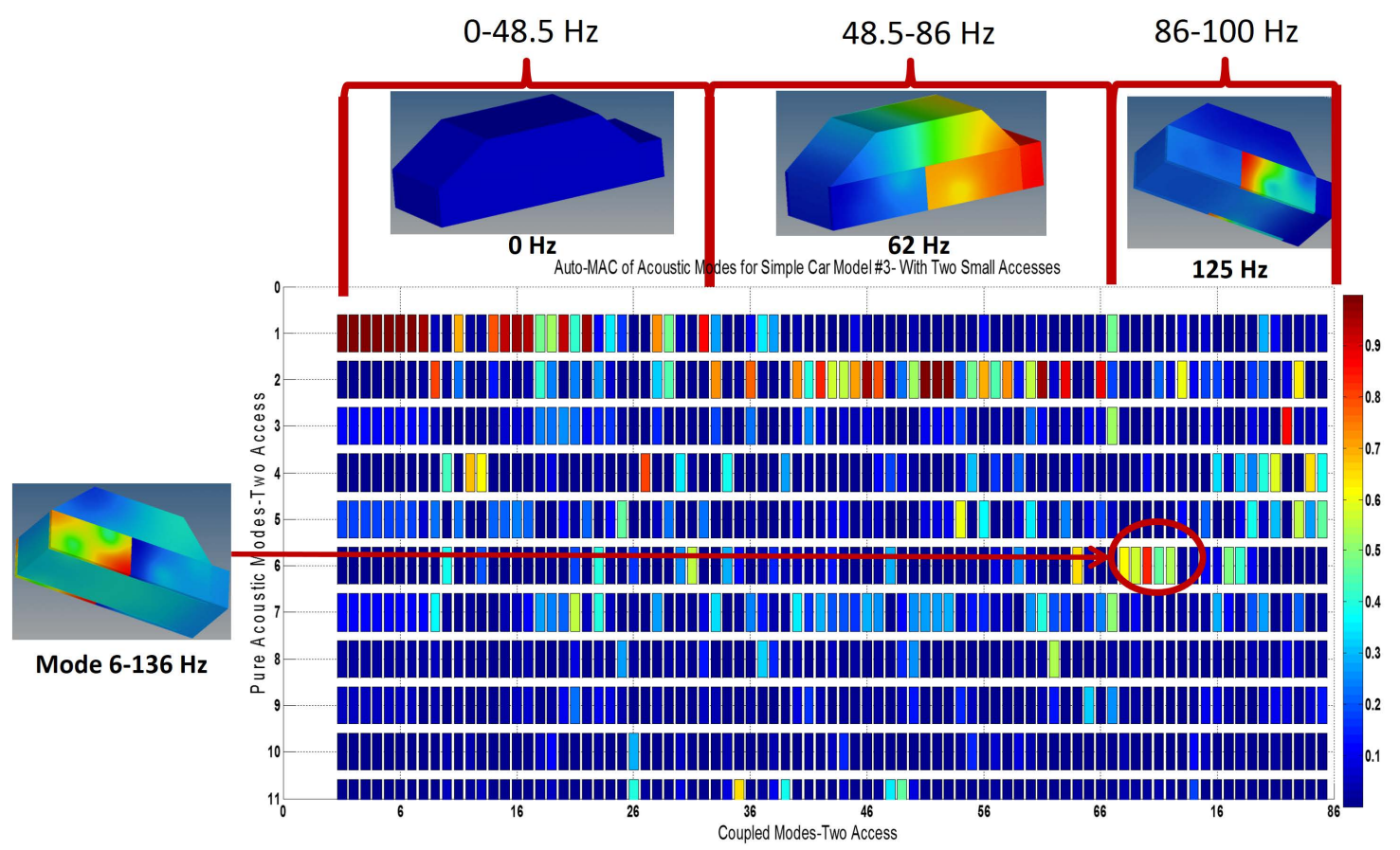

Figure 61: MAC plot between SCM \#3B pure acoustic modes and SCM \#3B coupled mode shapes

Figure 61 shows the MAC plot between SCM \#3B pure acoustic and coupled acoustic modes. As shown, the $0 \mathrm{~Hz}$ pure acoustic mode participates in the coupled acoustic modes from $0 \mathrm{~Hz}-48.5 \mathrm{~Hz}$ and second acoustic mode at $62 \mathrm{~Hz}$ participates from $48.5 \mathrm{~Hz}$ to 86 $\mathrm{Hz}$. Also, pure acoustic mode 6 at $136 \mathrm{~Hz}$ participates in the $86 \mathrm{~Hz}-100 \mathrm{~Hz}$ range. Thus, it shows that high frequency pure acoustic modes also participate in the low frequency coupled acoustic modes.

\subsubsection{Effect of door cavities on acoustic mode shapes}

Simple Car Models 2A, 3A and 3B are compared to understand the effect of modeling different types of door cavities on the natural frequencies and modes shapes of the acoustic meshes of the simple car models. Simple Car Model \#2B is not compared as its structural mesh is different thus leading to different coupled acoustic modes. 
Table 10: Comparison of acoustic natural frequencies for SCM \#2A, SCM \#3A and SCM \#3B

\begin{tabular}{|c|c|c|}
\hline $\begin{array}{c}\text { Simple Car Model \#2A- } \\
\text { No Door Cavities (Hz) }\end{array}$ & $\begin{array}{c}\text { Simple Car Model \#3A- } \\
\text { One Large Access (Hz) }\end{array}$ & $\begin{array}{c}\text { Simple Car Model \#3B- } \\
\text { Two Small Accesses (Hz) }\end{array}$ \\
\hline $1.84 \mathrm{E}-06$ & $3.21 \mathrm{E}-06$ & 0 \\
\hline 64.07 & 61.38 & 62.073 \\
\hline & 102.87 & \\
\hline & 105.65 & 111.87 \\
\hline 113.90 & 111.65 & 125.34 \\
\hline & 123.34 & 127.09 \\
\hline & 130 & 136.17 \\
\hline & 149.73 & 146.06 \\
\hline & & 151.86 \\
\hline 153.06 & 153.53 & 161.77 \\
\hline 154.94 & 159.17 & \\
\hline
\end{tabular}

Table 10 shows the acoustic natural frequencies of the SCM \#2A, SCM\#3A and SCM\#3B. All the natural frequencies marked in gray have similar mode shapes. As shown, SCM \#3A has one access and that introduces two additional acoustic modes at $102.8 \mathrm{~Hz}$ and 105.6 $\mathrm{Hz}$. Thus, additional acoustic modes are introduced due to the presence of door cavities. Also, for simple car models with door cavities (i.e. SCM \#3A and SCM \#3B) the first natural frequency decreases from $64 \mathrm{~Hz}$ to $61 \mathrm{~Hz}$ and $62 \mathrm{~Hz}$ respectively. Thus, the number of accesses has an effect at lower frequencies, but at higher frequencies the one and two accesses models don't show significant difference.

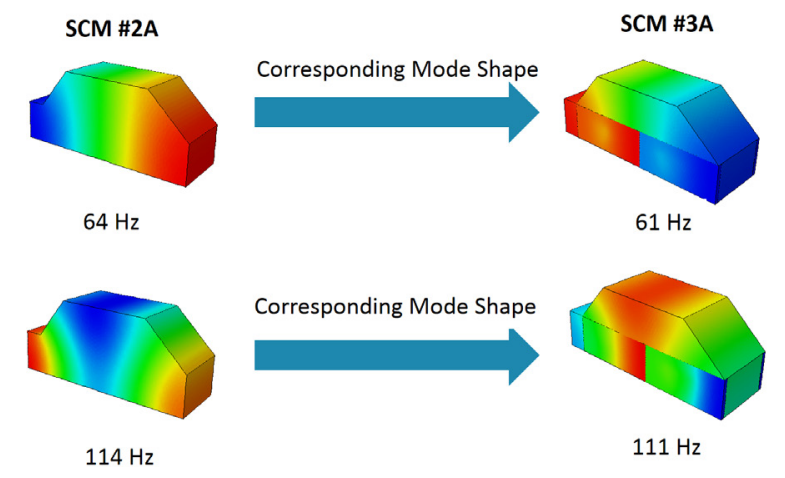

Figure 62: Mode shape comparison between SCM \#2A and SCM \#3A

Figure 62 shows the mode shape comparison for SCM \#2A and SCM \#3A. As shown, the overall mode shapes are the same even after the addition of door cavities but the natural frequencies decrease from $64 \mathrm{~Hz}$ to $61 \mathrm{~Hz}$ and from $114 \mathrm{~Hz}$ to $111 \mathrm{~Hz}$. Thus, due to the addition of acoustic cavities the car cavity becomes acoustically longer resulting in decrease in natural frequency. 


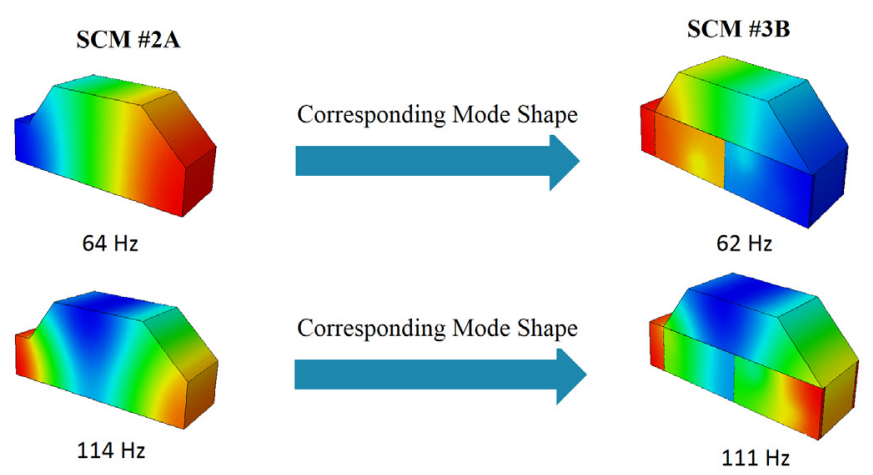

Figure 63: Mode shape Comparison between SCM \#2A and SCM \#3B

Figure 63 shows the mode shape comparison between SCM \#3A and SCM \#3B. As shown, the first two natural frequencies for both the models have the same mode shapes but the natural frequencies decrease for these modes as the acoustic cavity length increases due to the addition of door cavities. The car becomes acoustically longer or wider as the route for the air to travel inside the door cavities is only through the accesses.

\subsubsection{Effect of door cavities on acoustic sensitivity at DRE}

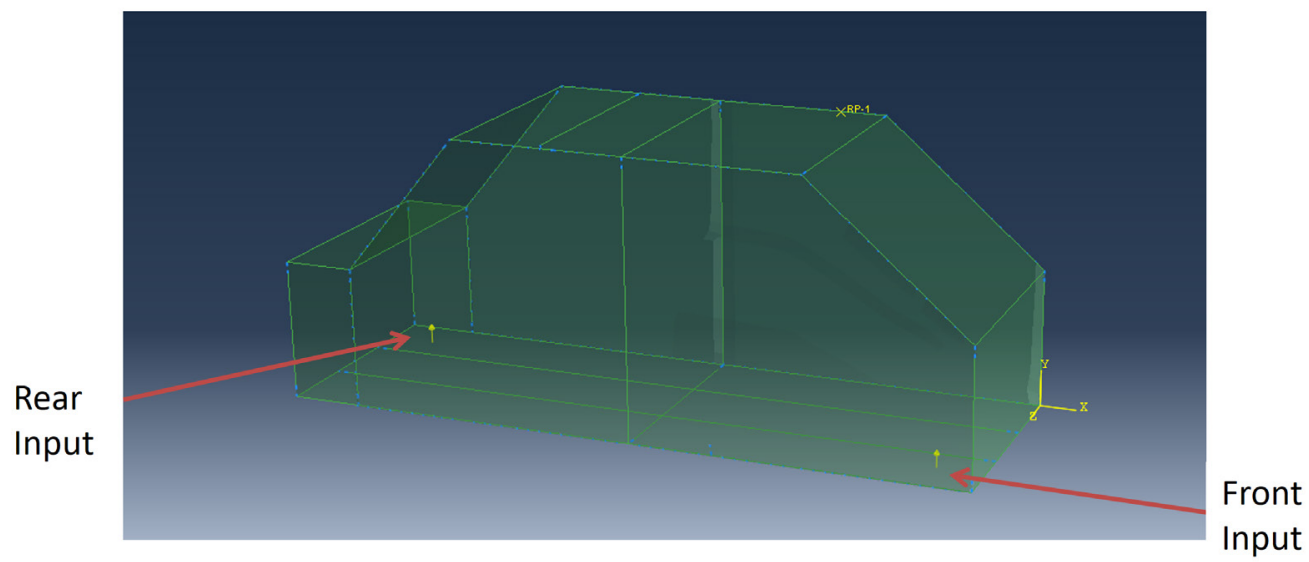

Figure 64: Structural Input Points for SCM \#2

Figure 64 shows the input points in SCM\#2 structural mesh. As shown two inputs are used, at front and rear end of the vehicle. These inputs mimic the shaker inputs during the full structural modal testing of Chevrolet Cruze LTZ body-in-white (BIW) model and a unit force in the Y- direction is applied at these points. As shown in Figure 64 and Figure 54 we have two inputs on the structural mesh of SCM $\# 2 \mathrm{~A}, 3 \mathrm{~A}, 3 \mathrm{~B}$ and $\mathrm{SCM} \# 2 \mathrm{~B}$ respectively and the output at acoustic sensitivity at driver's right ear (DRE) is evaluated. The coordinates of DRE are same for all four acoustic models. 


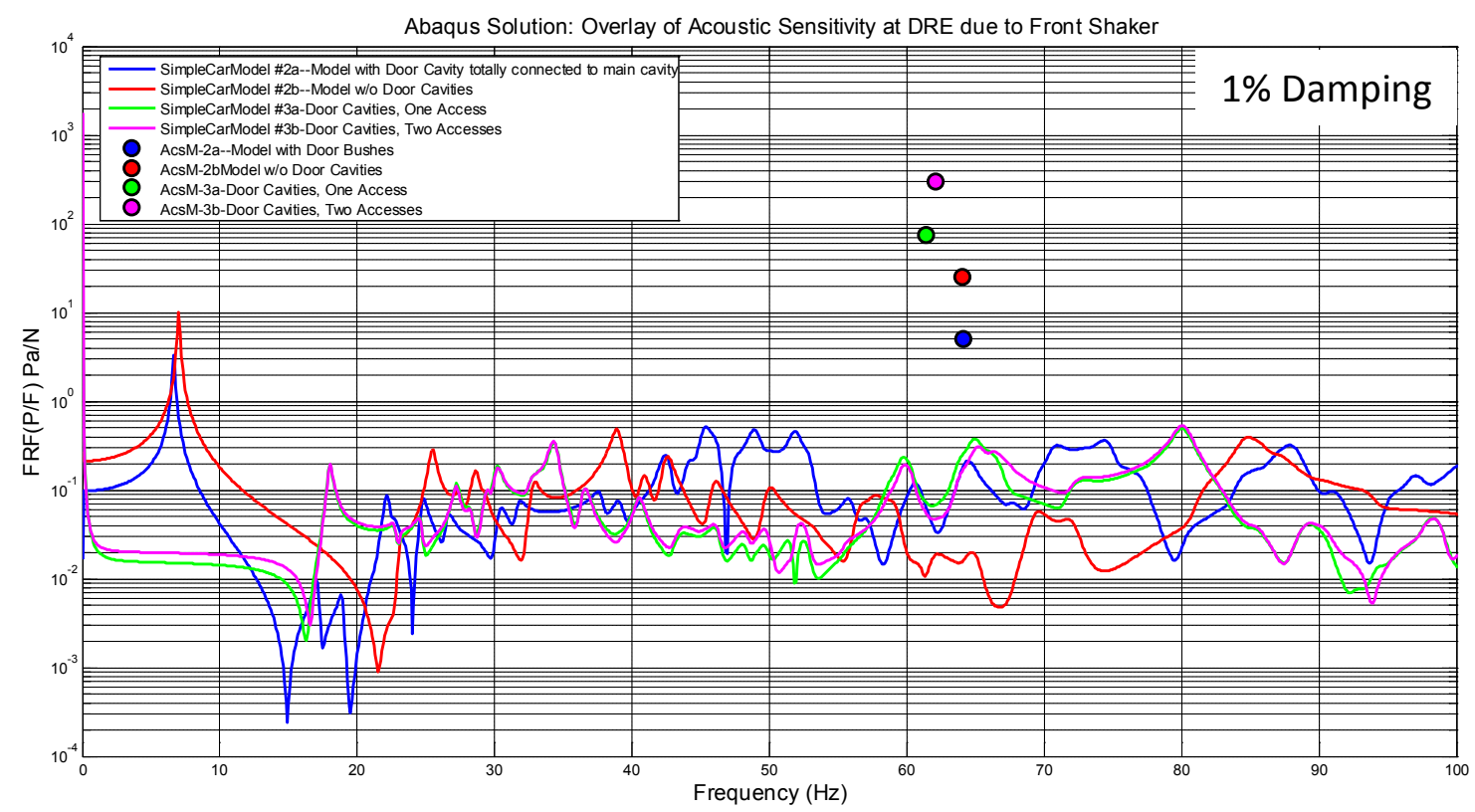

Figure 65: Comparison of acoustic sensitivity at DRE due to front input for SCM \#2A, \#2B, \#3A and \#3B

Figure 65 shows acoustic sensitivity at DRE due to front input on the structural mesh. Circular markers on the top represent second pure acoustic natural frequency for SCM 2A, 2B, 3A and 3B. As shown 1\% modal damping is added while calculating these FRF's. The FRF's for SCM\#3A and 3B overlap each other over the entire frequency range, thus it is concluded that number of door accesses does not matter for the acoustic sensitivity at DRE if the total area of opening remains the same. At $40 \mathrm{~Hz}$, trend for SCM \#2B and SCM \#2A flips with the SCM \#2A acoustic sensitivity higher up to $100 \mathrm{~Hz}$, but the magnitude of SCM \#2B is higher below $40 \mathrm{~Hz}$. Also, the second pure acoustic mode for SCM with door cavities (i.e. SCM \#3A and SCM \#3B) is marginally lower as compared to SCM without door cavities (i.e. SCM \#2A and SCM \#2B). 


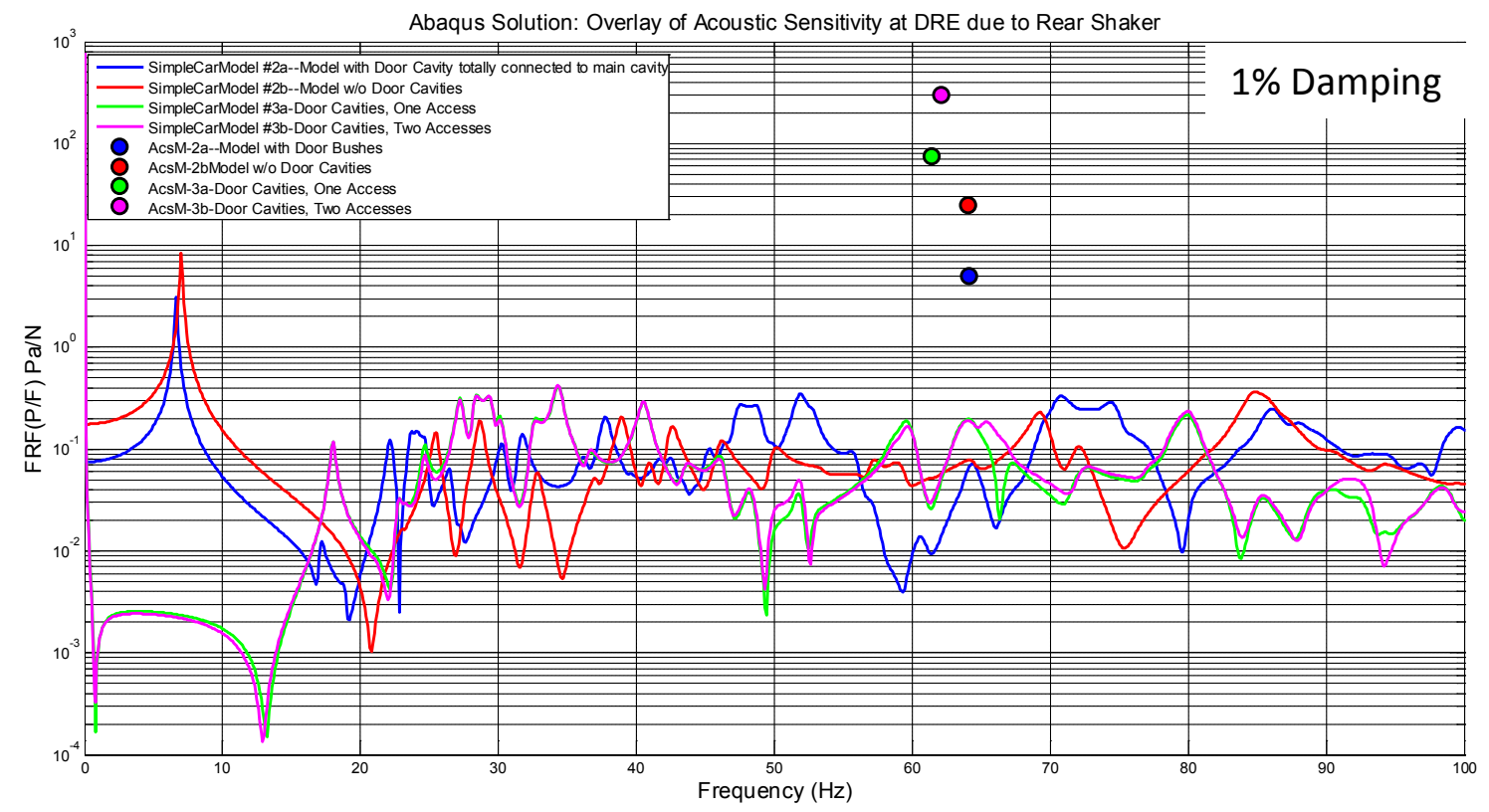

Figure 66: Comparison of acoustic sensitivity at DRE due to rear input for SCM \#2A, \#2B, \#3A and \#3B

Figure 66 shows the acoustic sensitivity at DRE between 0 to $100 \mathrm{~Hz}$. As shown, SCM \#3A and SCM \#3B curves overlap each other, indicating that number of access is inconsequential at DRE if the total area of opening is constant. Also, all the models with door cavities (i.e. SCM \#3A and SCM \#3B) and SCM \#2A and SCM \#2B show different trends throughout the frequency range. Thus, looking at the above observations, it can be concluded that door cavities act as Helmholtz resonators and change the natural frequencies of the cavity leading to additional acoustic modes.

\subsection{Simple Car Model \#4 (SCM\#4)}

Simple Car Model \#4 is modeled to observe the effect of rear seats and speaker openings on the rear package shelf on the acoustic boom phenomenon.

\subsubsection{Simple Car Model \#4A (SCM \#4A)}

SCM \#4A structural mesh: The same structural mesh as for SCM \#2A is used for analysis.

SCM \#4A Acoustic Mesh: Simple car model \#4 is modeled to observe the effect of rear shelf speaker opening area and rear seats on the acoustic boom phenomenon. The major geometric dimensions are same as in simple car model \#2A. 


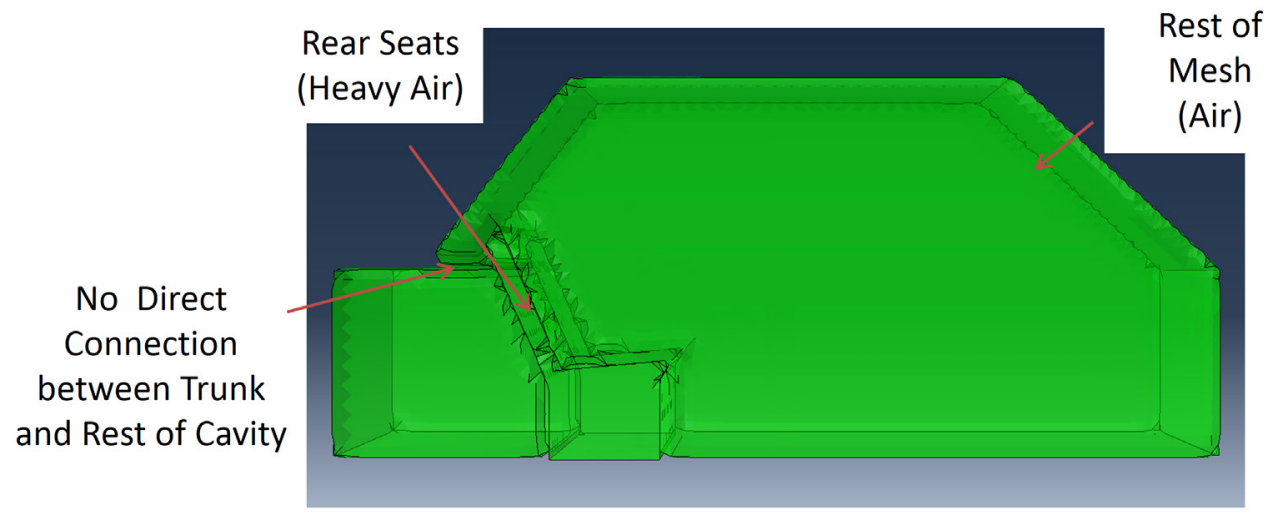

Figure 67: SCM \#4A Acoustic Mesh

Figure 67 shows the SCM \#4A acoustic mesh. As shown, the rear seats are modeled as heavy air, thus separating the trunk from the rest of acoustic cavity. The rear seat dimensions are similar to the rear seat in Chevrolet Cruze LTZ. There is no direct connection between the rest of the cavity and trunk and the only way for the air to enter the trunk is through the rear seats.

Table 11: Material properties of SCM \#4A

\begin{tabular}{|l|l|}
\hline Air & $\begin{array}{l}\text { Density }-1.225 \mathrm{~kg} / \mathrm{m}^{3} \\
\text { Bulk Modulus }-141610 \mathrm{~Pa}\end{array}$ \\
\hline Heavy Air & $\begin{array}{l}\text { Density }-60 \mathrm{~kg} / \mathrm{m}^{3} \\
\text { Bulk Modulus }-1350000 \mathrm{~Pa}\end{array}$ \\
\hline
\end{tabular}

Table 12: Mesh attributes of SCM\#4B

\begin{tabular}{|l|l|}
\hline Number of Nodes & 113050 \\
\hline Number of Elements & 527478 \\
\hline Type of Elements & 4-noded Tetra \\
\hline
\end{tabular}

Table 11 shows the material properties of different materials used in SCM \#4A and Table 12 shows the mesh attributes for SCM\#4A.

\subsubsection{Effect of addition of rear seats and rear package shelf speaker openings on Eigen solution of simple car models:}

The differences between SCM\#2A and SCM \#4A are rear seats and speaker openings on the rear package shelf. 
Table 13: Natural frequency comparison for SCM \#2A and SCM \#4A

\begin{tabular}{|c|c|c|}
\hline Mode No. & SCM \#2A & SCM \#4A \\
\hline 1 & $64.07 \mathrm{~Hz}$ & $34.32 \mathrm{~Hz}$ \\
\hline 2 & $113.9 \mathrm{~Hz}$ & $79.8 \mathrm{~Hz}$ \\
\hline 3 & $153.06 \mathrm{~Hz}$ & $136.7 \mathrm{~Hz}$ \\
\hline 4 & $155.02 \mathrm{~Hz}$ & $150.5 \mathrm{~Hz}$ \\
\hline
\end{tabular}

Table 14 shows the natural frequency comparison between SCM \#2A and SCM \#4A. As shown, due to the addition of rear seats in SCM \#4A the second pure acoustic mode drops from $64.08 \mathrm{~Hz}$ in SCM \#2A to $34.32 \mathrm{~Hz}$ in SCM \#4A and third pure acoustic mode drops from $113.9 \mathrm{~Hz}$ to $79.8 \mathrm{~Hz}$ and so on till the fifth pure acoustic modes after which both the models have relatively same natural frequencies. Thus, the addition of rear seats and speaker openings reduces the pure acoustic mode frequencies in the boom frequency range.

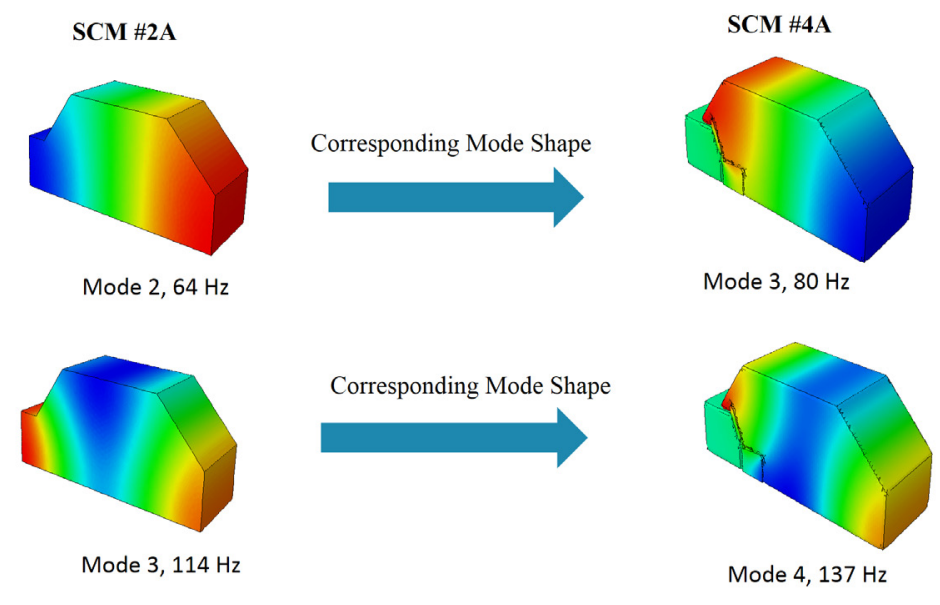

Figure 68: Mode shape comparison between SCM \#2A and SCM \#4A

Figure 68 compares the mode shapes between SCM\#2A and SCM \#4A. As shown, natural frequencies increase due to the addition of seats for the same mode shape as acoustic length of the car decreases. Also, the trunk has a lower acoustic sensitivity as compared to rest of the cavity indicating that the rear seats act as acoustic barriers, which is in agreement with the result obtained in Chevrolet Cruze analysis.

4.4.3. Simple Car Model \#4B (SCM \#4B)

SCM \#4B Structural Mesh: Same as Simple Car Model \#2A. 
SCM \#4B Acoustic Mesh: Simple Car Model \#4B is modeled same as SCM \#4A but there is a direct connection between rest of acoustic cavity and the trunk through the speaker holes.

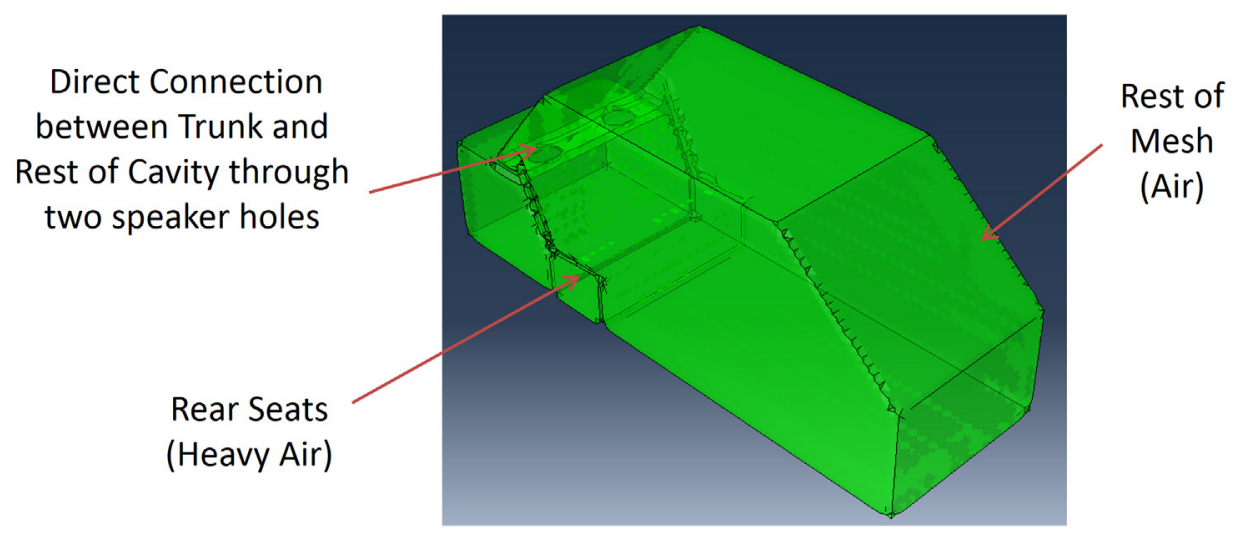

Figure 69: SCM \#4B Acoustic Mesh

Figure 69 shows the SCM \#4B acoustic mesh. As shown, the speaker holes provide a direct connection between trunk cavity and rest of acoustic cavity. The diameter of speaker holes is $182.86 \mathrm{~mm}$.

Table 14: Mesh Attributes of SCM \#4B

\begin{tabular}{|l|l|}
\hline Number of Nodes & 112282 \\
\hline Number of Elements & 524081 \\
\hline Type of Elements & 4-noded Tetra \\
\hline
\end{tabular}

Table 13 shows the mesh attributes of SCM \#4B. Simple car model \#4B has same material properties as SCM \#4A. The structural and acoustic meshes of the SCM \#4B, coupled using tie constraints are solved in Abaqus using the 'steady state modal' step for acoustic sensitivity at driver's right ear (DRE).

\subsubsection{Effect of addition of speaker openings on acoustic sensitivity at DRE}

SCM \#4A and SCM \#4B acoustic meshes are compared to understand the difference in acoustic sensitivity at driver's right ear due to the structural input at the front and rear of the vehicle. The front and rear structural inputs are shown in figure 64. Similar to SCM \#3A and 3B the acoustic meshes are coupled with the structural meshes and solved in Abaqus. 


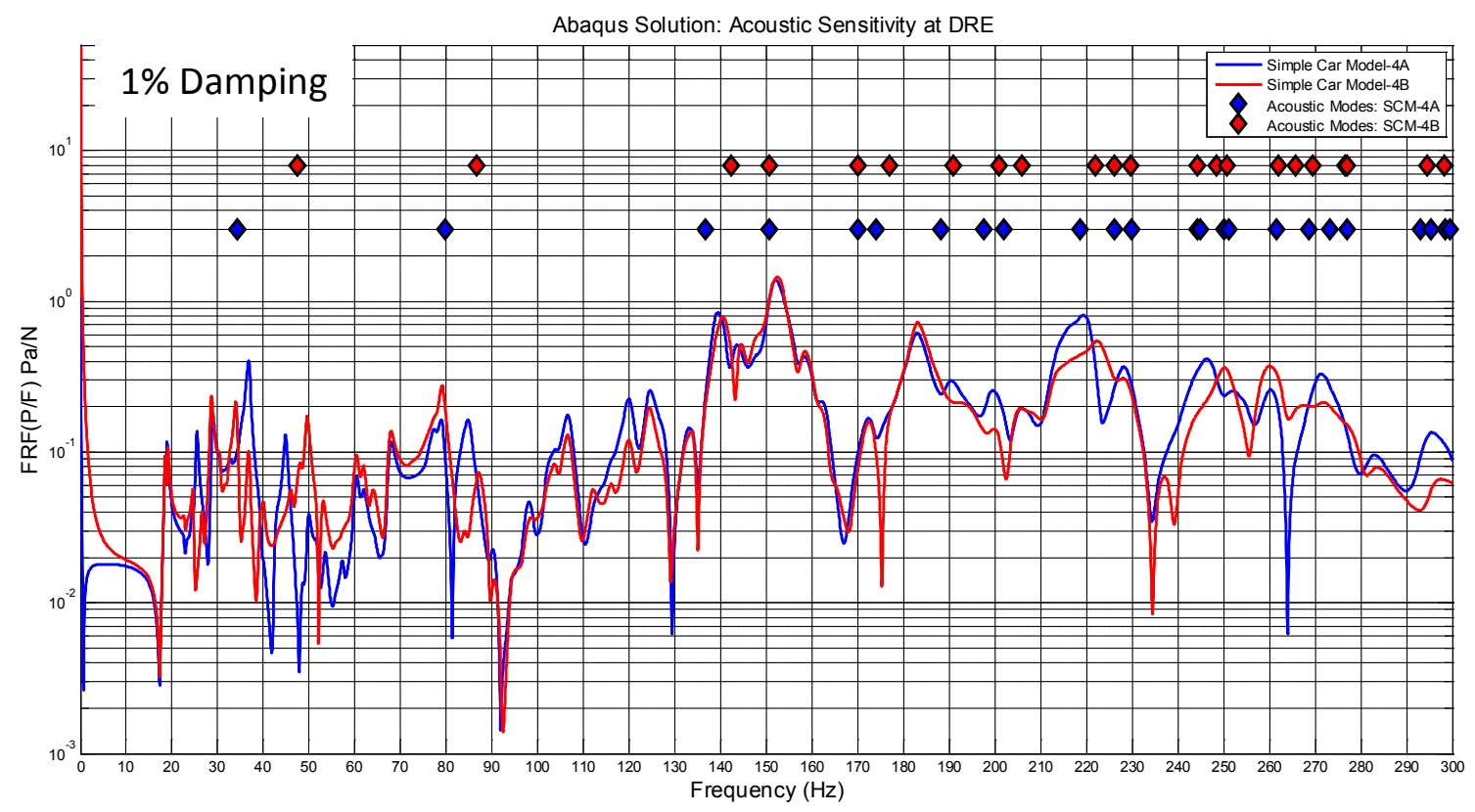

Figure 70: Acoustic sensitivity at DRE due to front input between SCM \#4A and SCM \#4B

Figure 70 shows the comparison of acoustic sensitivity at DRE due to front input on the structural mesh from 0 to $300 \mathrm{~Hz}$. As shown, at higher frequencies there is no difference between SCM\#4A and SCM\#4B but at the frequencies from $20 \mathrm{~Hz}-80 \mathrm{~Hz}$ there is a large difference between acoustic sensitivities. Thus, the speaker openings influence the acoustic response at DRE at lower frequencies. The blue and red spade markers at top represent the pure acoustic modes for SCM\#4A and SCM\#4B. Also, due to the addition of speakers the natural frequency of pure acoustic mode shifts higher in frequency for the first three modes. 


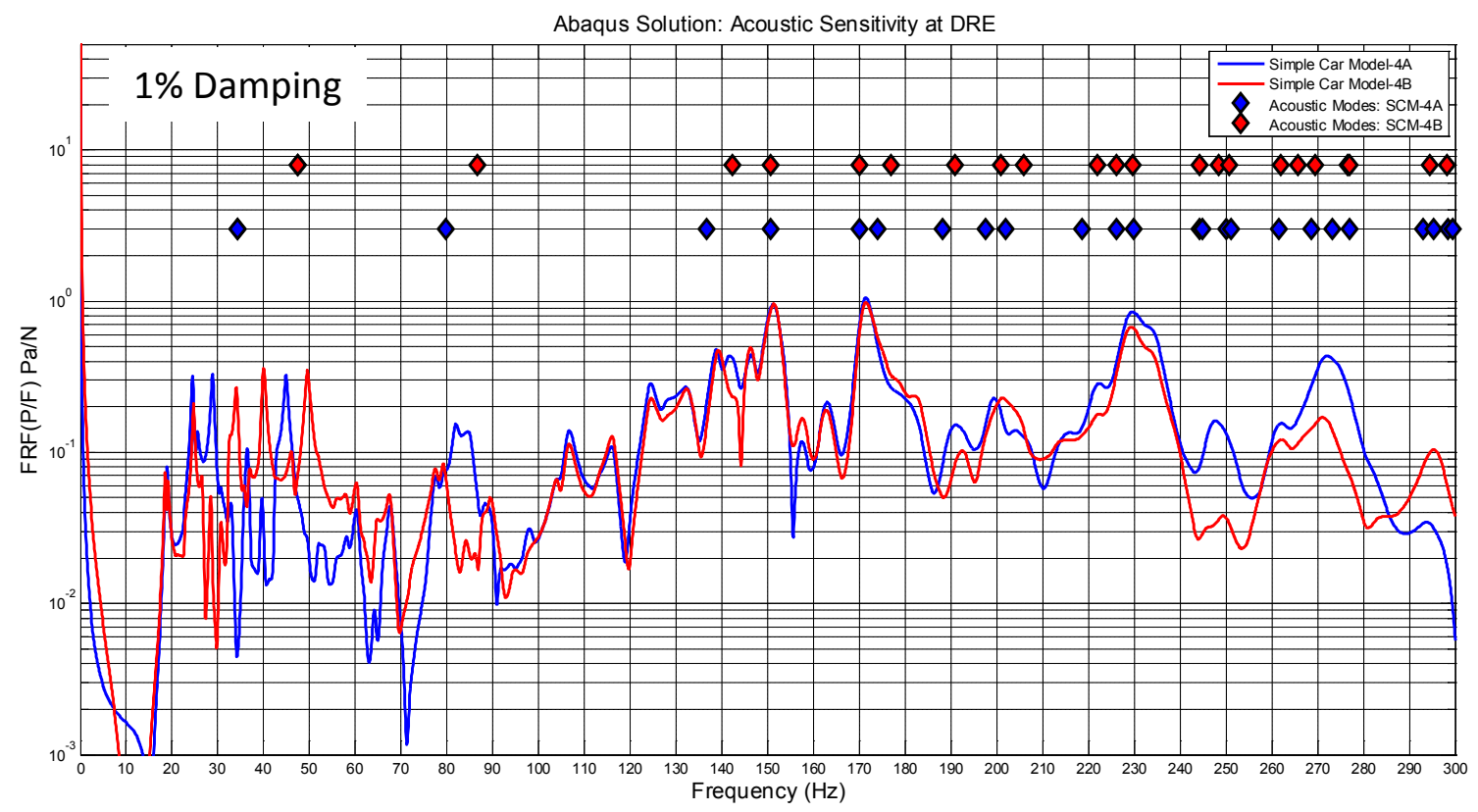

Figure 71: Acoustic sensitivity at DRE due to rear input between SCM \#4A and SCM \#4B

Figure 71 shows the acoustic sensitivity at DRE for SCM \#4A and SCM \#4B due to rear input. Similar to the front input response, there is a large difference in lower frequencies from $20-70 \mathrm{~Hz}$ due to the addition of speaker openings.

\subsubsection{Simple Car Model \#4C (SCM \#4C)}

Now, we know that speaker openings affect the acoustic sensitivity at DRE and so we vary the speaker diameter openings measure its effect on the same.

SCM \#4C Structural Mesh: Same as structural mesh for SCM \#2A.

SCM \#4C Acoustic Mesh: SCM \#4A Acoustic mesh is the same as SCM \#4C with smaller speaker diameter.
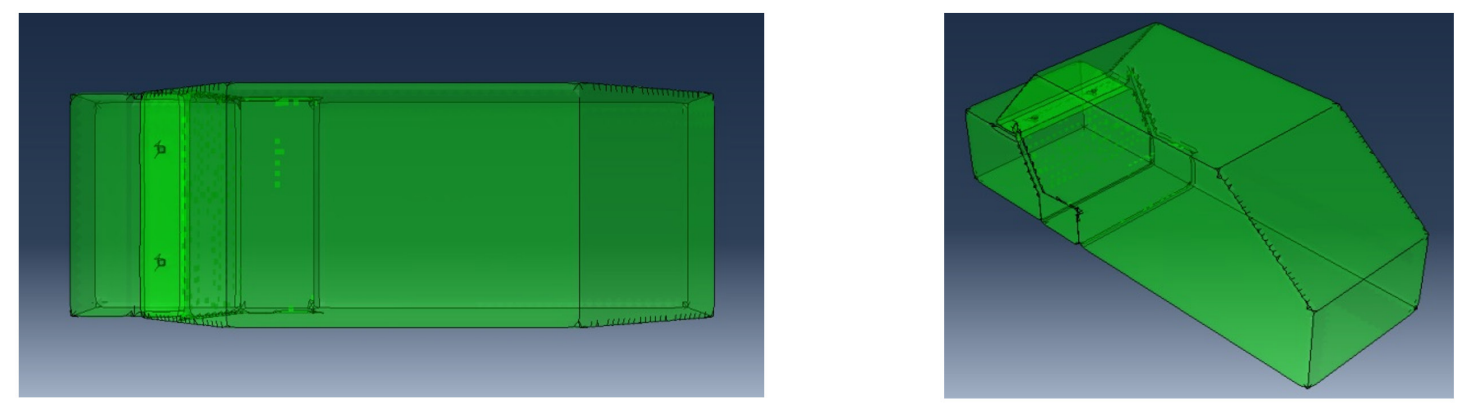

Figure 72: Different views of SCM \#4C Acoustic Mesh 
Figure 72 shows the different views of SCM \#4C acoustic Mesh. As shown, the speaker openings have a very small diameter of $44.7 \mathrm{~mm}$. SCM \#4C models the lower extreme of speaker opening diameter and is created to see the change in acoustic sensitivity at DRE due to a very small speaker opening.

Table 15: SCM \#4C Acoustic Mesh attributes

\begin{tabular}{|l|l|}
\hline Number of Nodes & 199640 \\
\hline Number of Elements & 927740 \\
\hline Type of Elements & 4-noded Tetra \\
\hline
\end{tabular}

Table 15 shows the different mesh attributes of SCM \#4C acoustic mesh with the material properties same as SCM \#4B.

\subsubsection{Simple Car Model \#4D (SCM \#4D)}

SCM \#4D Structural Mesh: Same as SCM \#2A structural mesh.

SCM \#4D Acoustic Mesh: SCM \#4D acoustic mesh is the same as SCM \#4C with a different speaker opening diameter.
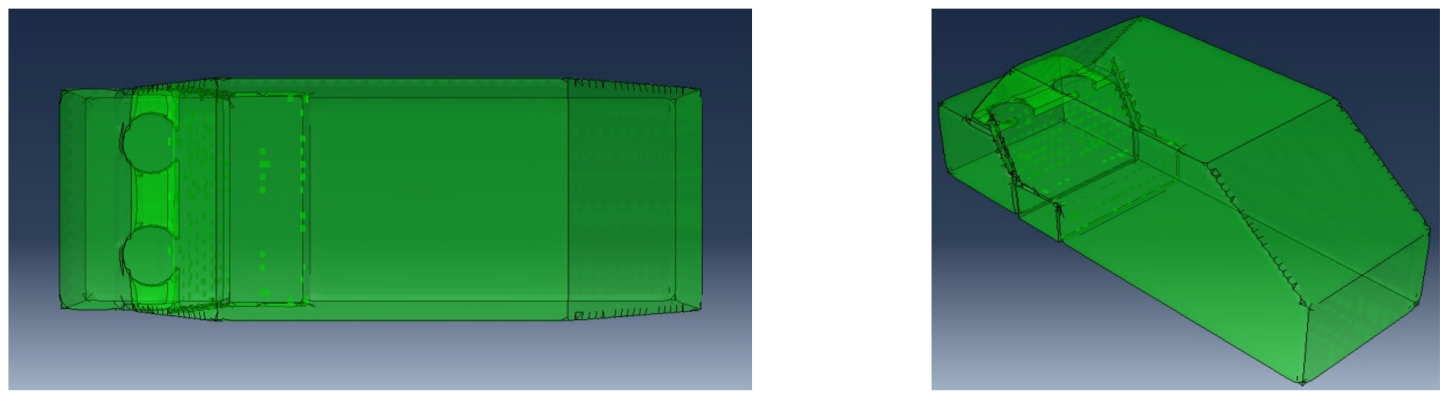

Figure 73: Different Views of SCM \#4D Acoustic Mesh

Figure 73 shows the SCM \#4D acoustic mesh. As shown, SCM \#4D acoustic mesh is similar to SCM \#4B but with very large speaker openiengs having diameter of $264 \mathrm{~mm}$. 
Table 16: SCM \#4D Acoustic Mesh attributes

\begin{tabular}{|l|l|}
\hline Number of Nodes & 112981 \\
\hline Number of Elements & 527493 \\
\hline Type of Elements & 4-noded Tetra \\
\hline
\end{tabular}

Table 16 shows the acoustic mesh attributes of SCM \#4D. The material properties of SCM \#4D are the same as SCM \#4B for the rear seats and rest of the cavity.

\subsubsection{Effect of different rear package shelf speaker opening diameter on the acosutic sensititvity at DRE}

SCM\#4B, 4C and 4D have different rear package speaker diameters and are compared with each other to evalaute the influence of speaker opening diameters on the acoustic sensitivity at DRE due to inputs on the structural mesh.

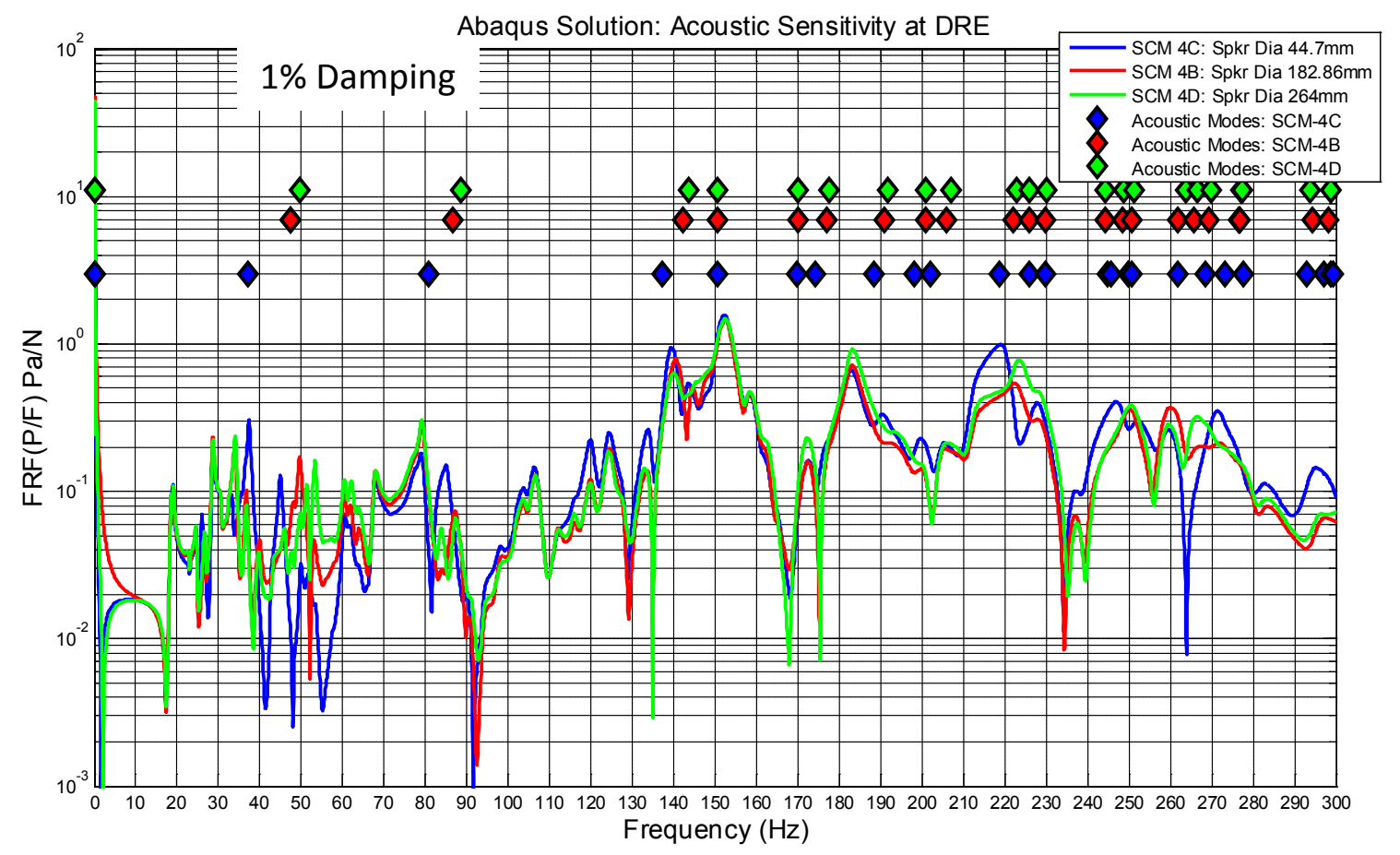

Figure 74: Comparison of acoustic sensitivity between SCM \#4B, \#4C and \#4D due to front input

Figure 74 shows the acoustic sensitivity at DRE due to the front input on structural mesh from $0-300 \mathrm{~Hz}$. As shown in the legend, SCM models are arranged from small to large speaker diameter openings and the spade markers on top represent the pure acoustic modes for these models. The graph and the spade markers are color coded and every simple car model is represented by a single color. In the boom frequency range of $20 \mathrm{~Hz}-70 \mathrm{~Hz}$ we have higher deviation of acoustic sensitivity at DRE for different speaker openings, but at 
higher frequencies they show the same response. Also, as the speaker diameters get smaller the natural frequency reduces for second, third and fourth modes.

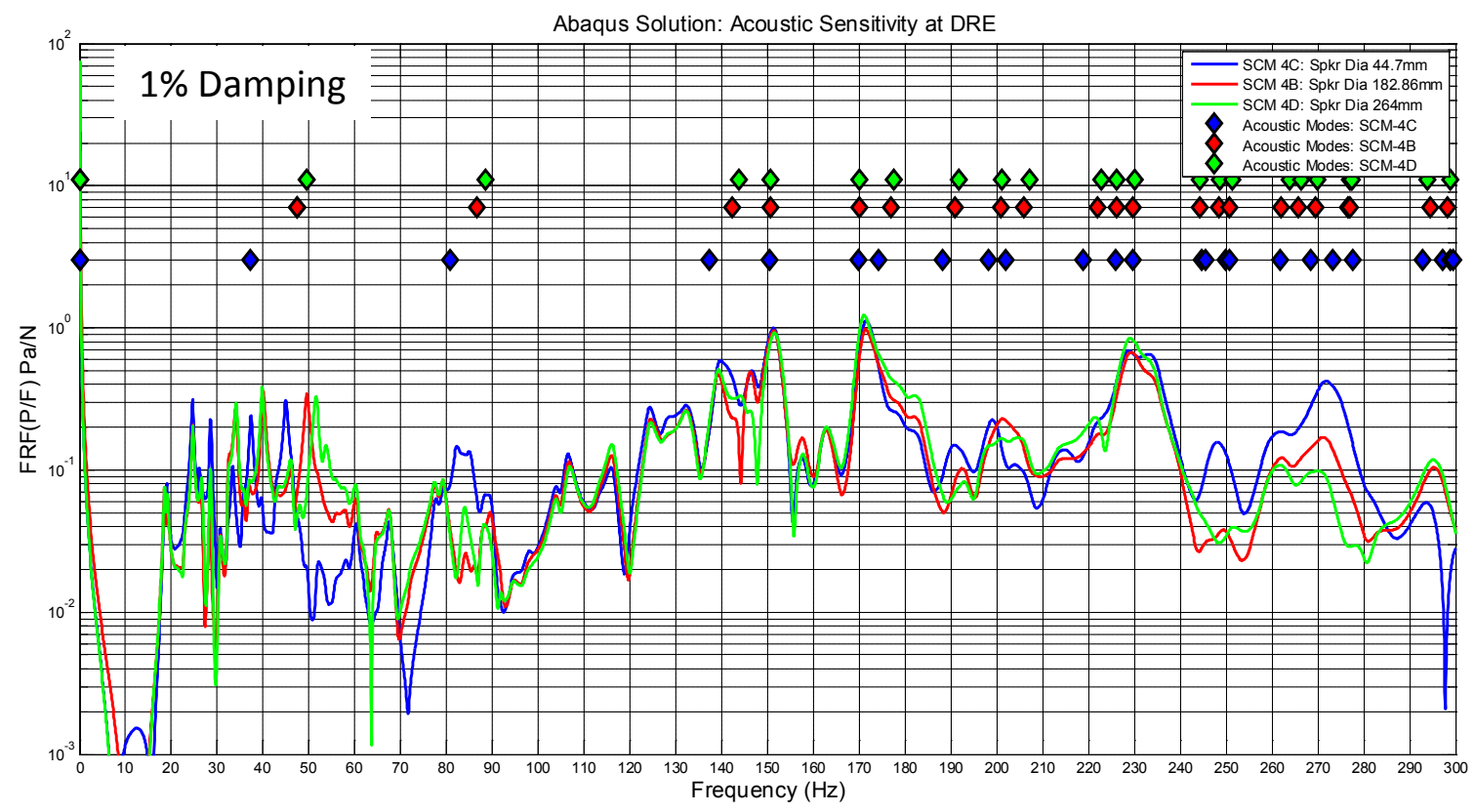

Figure 75: Comparison of acoustic sensitivity between SCM \#4B, \#4C and \#4D due to rear input

Figure 75 shows the comparison of acoustic sensitivity at DRE due to rear input. Same as the front input results, we have a deviation in response between $30 \mathrm{~Hz}$ to $100 \mathrm{~Hz}$. Thus, speaker diameters also influence the acoustic sensitivity at DRE.

In order to study the physics of the role of speaker openings a spring-mass-damper system is proposed.

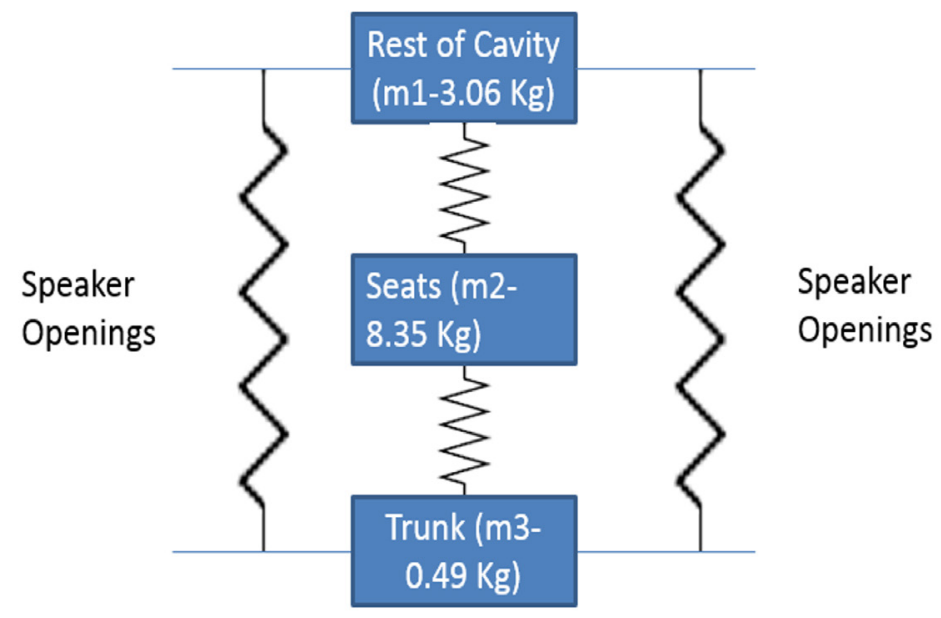

\section{$\underline{\mathrm{SCM} 4 \mathrm{~B}}$}

Figure 76: Simplified figure showing role of speaker openings for SCM \#2B 
Figure 76 shows a simple spring-mass-damper system. As shown, the speakers act as additional springs that connect rest of the cavity and trunk besides the seats. Thus, the speaker openings act as stiffeners and seats acts as a mass that lies between the mass of rest of cavity and trunk.

\subsection{Pick-Up Truck Modelling}

A pick-up truck was modeled as a secondary project to study structural-acoustic coupling and transfer of energy between structural and acoustic meshes in Abaqus 6.12-3. The pickup truck was modeled, meshed and solved in Abaqus. It was modeled to study the acoustic response at driver's right ear (DRE) for an input on the truck bed. Here, the air gap between the truck cabin and truck bed is modeled as a closed box. Figures below show the various characteristics of the pick-up truck model.

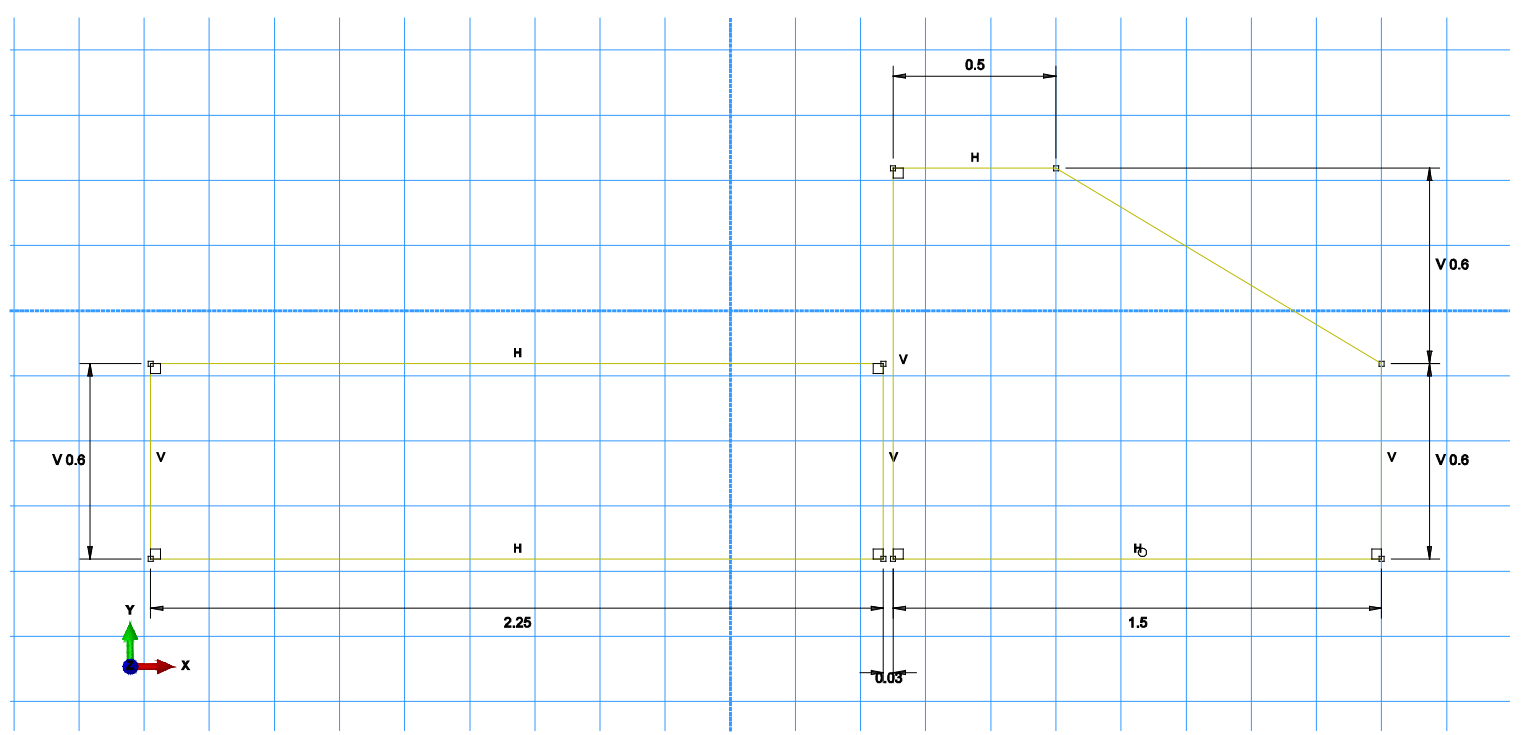

Figure 77: Dimensions in meters $(m)$ of Simple Pick-Up Truck Model 


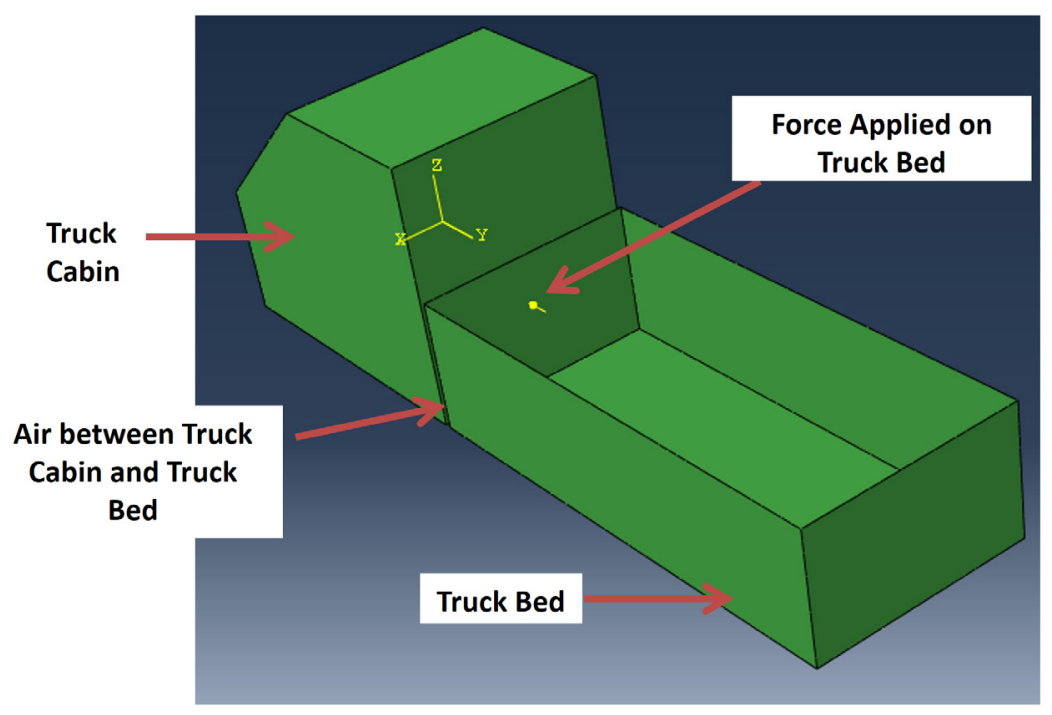

Figure 78: Pick-Up Truck model characteristics

Figure 77 and 78 show the pick-up truck model parameters. Elements linear shell quadrilateral (S4R) and linear tetrahedral (AC3D4) are used to mesh the pick-up truck with 14841 total number of elements. As seen in figure 78 there is an air gap between the truck bed and truck cabin. Also, there is acoustic mesh inside the truck cabin and force is applied on the truck bed and acoustic sensitivity is measured at the driver's right ear (DRE).

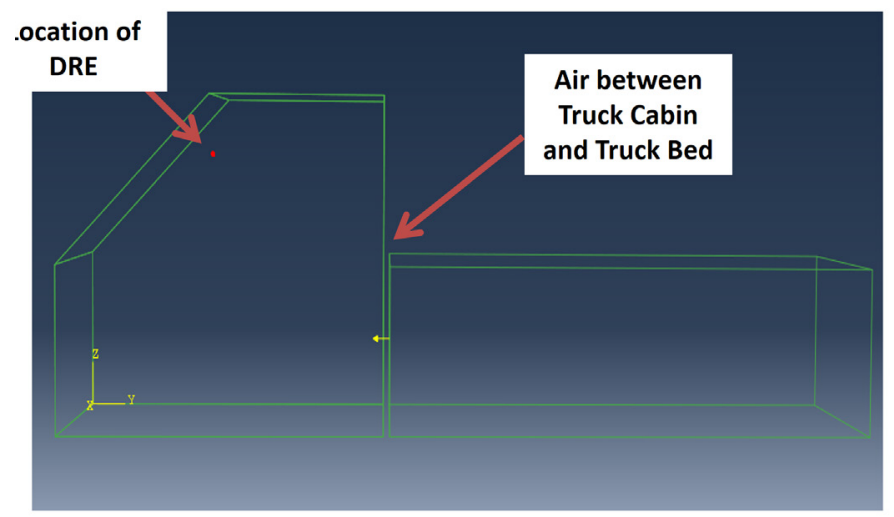

Figure 79: Pick-Up Truck model showing the structural input and acoustic output locations

Figure 79 shows the input and output locations on the pick-up truck model. Here the air between the between the truck bed and cabin is modeled as a closed box i.e. with no vent to the atmosphere. Thus, the energy is transferred from structure (truck bed) to air (between truck bed and cabin) to structure (truck cabin) and finally to the air (inside truck cabin) again. 


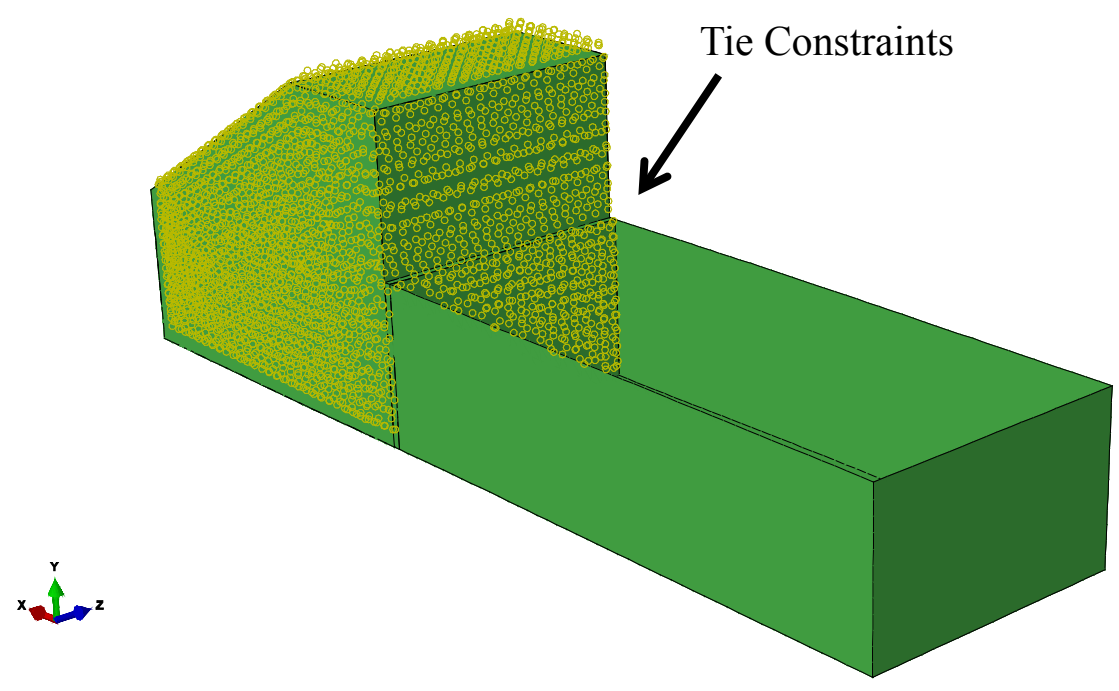

Figure 80: Tie Constraints for coupling on the pick-cup truck model

Figure 80 shows the tie constraints applied on the pick-up truck model to couple the structural mesh of truck bed to acoustic mesh of air gap and then coupling the air gap to the structural mesh of truck cabin. A tolerance value of $20 \mathrm{~mm}$ is applied for the tie constraints.

In Abaqus, a 'frequency step' is defined for the coupled pick-up truck model to evaluate the natural frequencies and mode shapes of the pick-up truck model. Then a 'Modal steady state dynamics' step is defined with $0 \%$ modal damping to evaluate frequency response functions(FRF's) on the pick-up truck model. 


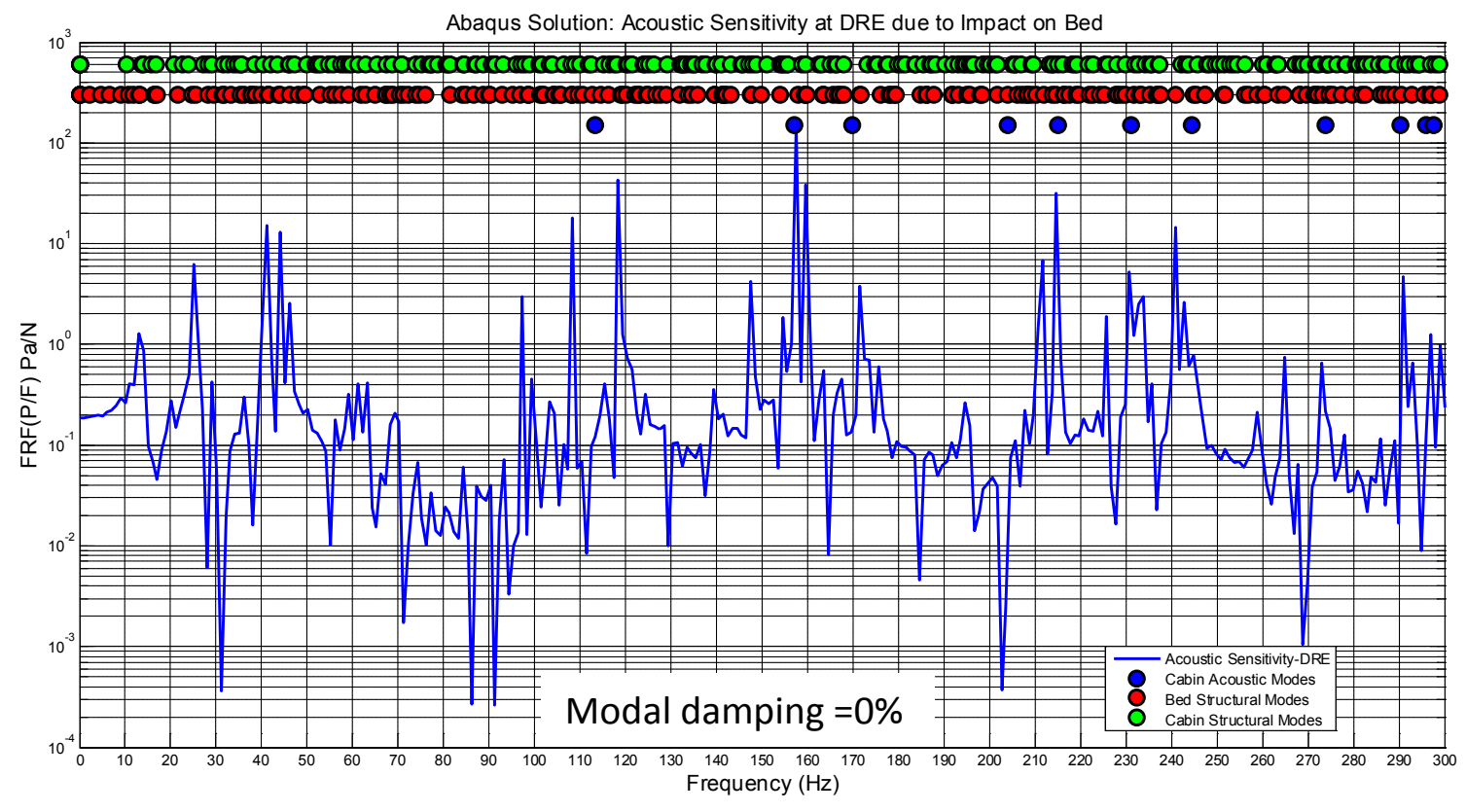

Figure 81: Acoustic sensitivity at DRE due to force on truck bed

Figure 81 shows the acoustic sensitivity at DRE from the force applied on truck bed with the blue circles representing the acoustic modes of the truck cabin and red and green circles representing the truck bed and cabin structural modes respectively. As shown, Abaqus effectively transfers the energy from the structural mesh to truck cabin acoustic mesh.

Now, in the previous model of pick-up truck, air gap is modeled is a closed box which is not the case in the actual pick-up truck where the air in the gap between the bed and cabin is free to interact with the atmosphere. To simulate this in Abaqus, acoustic infinite elements are added on the closed boundary surface of the air gap and act as vent to the atmosphere. Also, soft springs were added on the truck bed, to get rid of additional rigid body modes. 


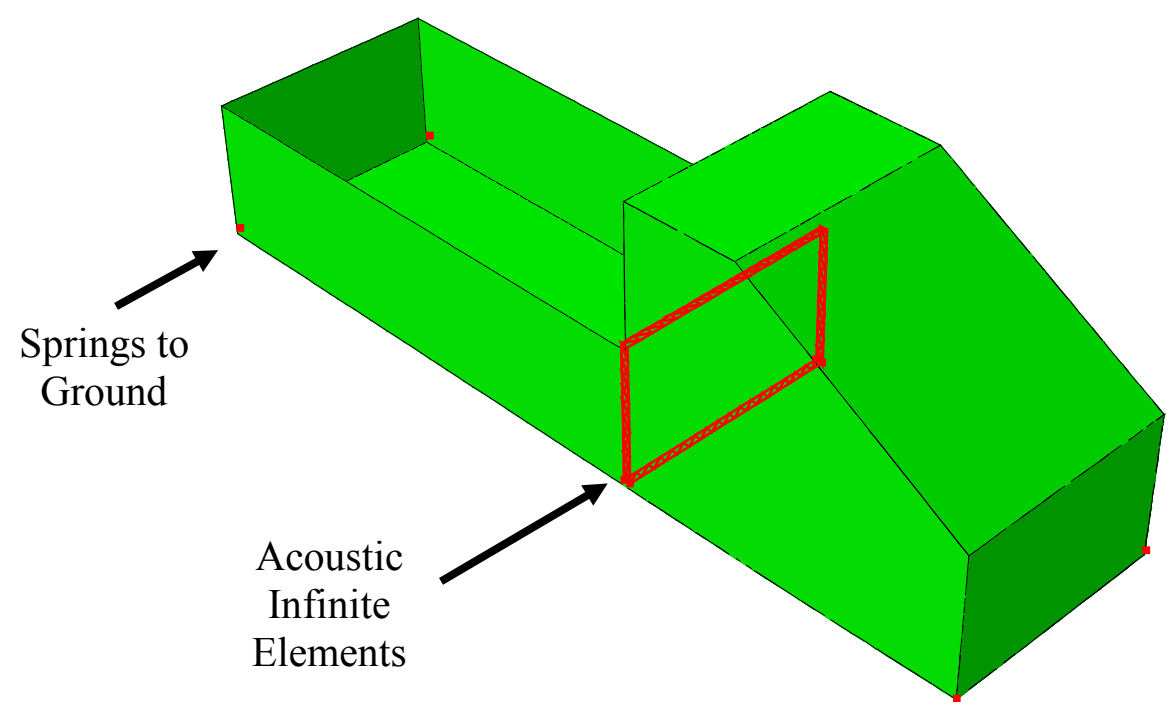

Figure 82: Pick-Up Truck modelling with acoustic infinite elements

Figure 82 shows the pick-up truck model with acoustic infinite elements. This model closely represents the actual conditions in a pick-cup truck. Also, air gap between the truck bed and cabin is varied and its effect on DRE is observed.

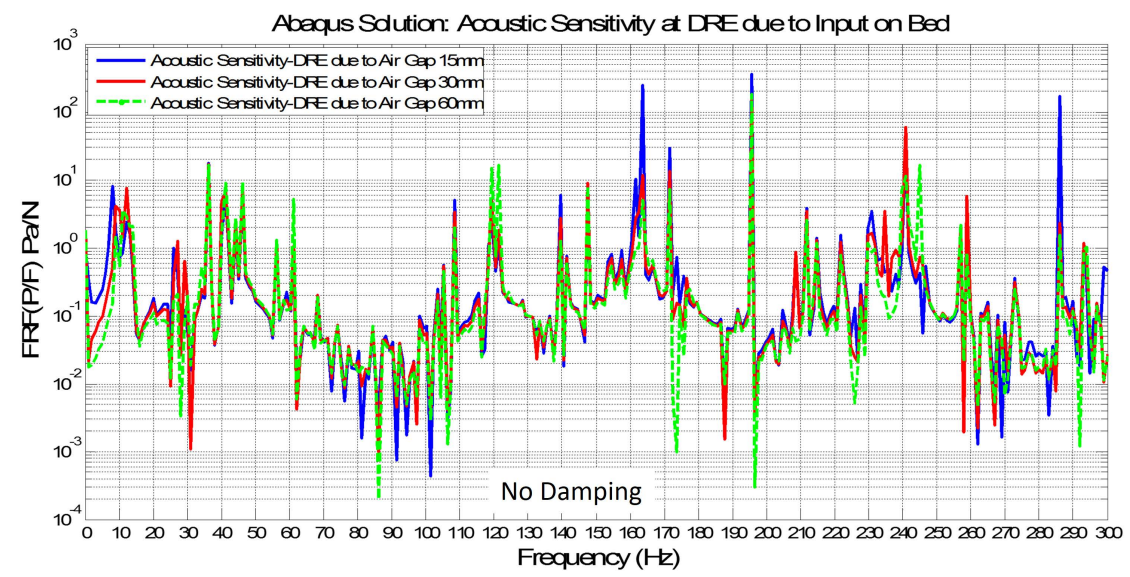

Figure 83: Acoustic Sensitivity at DRE for different air gap thickness values

Figure 83 shows the acoustic sensitivity at DRE due to varying air gap thickness with the air gap open to atmosphere. As shown, there is a large difference between DRE response for air gap of $15 \mathrm{~mm}$ and $60 \mathrm{~mm}$ in the frequency range of 0 to $30 \mathrm{~Hz}$ and at $170 \mathrm{~Hz}$. So, it can be concluded that air gap between truck and bed had a significant affect at some frequencies and in some frequencies no change is observed for the acoustic response at DRE. 


\section{5: Summary and Conclusions}

The purpose of this project was to investigate the fundamental physics of the acoustic boom phenomena, and provide specific recommendations on modeling techniques to improve model prediction accuracy. Specific goals were to study the modelling of vibro-acoustic coupling in Abaqus and subsequent application to the Chevrolet Cruze LTZ finite element model.

Finite element analysis of the structural-acoustic model of Chevrolet Cruze LTZ was carried out using Abaqus 6.12-3. Simple Car Models (SCM) were used to study the acoustic boom phenomenon and the effect of door and trunk cavities on it. Also, full structural and acoustic modal testing was performed on the Chevrolet Cruze LTZ BIW model to validate the finite element results.

Abaqus "strongly coupled" solution was found to make predictions consistent with the vibro-acoustic theory and the "strongly-coupled" solution method is recommended to predict automotive interior sound performance. The "strongly-coupled" solution successfully predicted acoustic performance sensitivity for changes to interior cabin configuration involving rear seatback position and inclusion of door cavities that were consistent with test results. Acoustic performance predictions using the "weakly-coupled" solution were inconsistent with test results. Also, it was found that rear seats tend to act as an acoustic barrier separating trunk cavity from the rest of the cavity. In order to effectively model the Chevrolet Cruze LTZ acoustic mesh a small gap should be modeled between the rear seat-back and trunk cavity as shown in Acoustic Mesh \#2.

Another key outcome of this work is new insight into the interpretation of acoustic modal participation. Traditionally, analysis of structure and acoustic cavity performance was performed separately and relationships were inferred. Vibro-acoustic issues were labeled and addressed as structure modes or acoustic modes. This work demonstrates that acoustic modes participate in many system modes and are not easily influenced by moving the natural frequency of one structure mode. Automotive vibro-acoustic performance tends to be robust to small changes in structural vibration characteristics. Significant change to poor vibro-acoustic performance is best accomplished by changing structure mode shapes to be less compatible with the acoustic cavity modes in the band of interest. This may involve a significant change in natural frequency.

A proposed vibro-acoustic interpretation graphic shows a mapping of pure acoustic cavity modes onto the coupled system vibro-acoustic modes. Calculation for this color map is essentially the MAC (modal assurance criterion) calculation between the uncoupled acoustic modes and the coupled system modes over the acoustic degrees of freedom. This graphic clearly shows frequency bands with the potential for high acoustic sensitivity and which coupled system modes contribute to the high sensitivity. In general, this graphic demonstrates that fundamental acoustic cavity modes participate in multiple coupled system modes. 
Simple car models were found to be effective in understanding the effect of smaller components on the acoustic boom phenomenon, which is not apparent in the huge Chevrolet Cruze LTZ model. SCM's offer significant insight into the design parameters for door cavity, trunk cavity and speaker openings and how they affect the acoustic response at driver's right ear. The door cavities make the car acoustically longer and introduce door cavity modes. The rear seats act as a high impedance path for the air with speaker's on the rear package shelf acting as a connection between the trunk and the rest of the cavity. Also, diameter of the speaker openings affects the acoustic response at DRE in the boom frequency range.

Also, a simple box model of a pick-up truck was analyzed in Abaqus. Using tie constraints for vibro-acoustic coupling, energy was transferred from a structural input to acoustic mesh and then from acoustic media to structural mesh and from the structural mesh to the driver's right ear in the acoustic cavity. A significant effect was observed on acoustic response at the DRE due to varying the air gap between the truck cabin and bed in the boom frequency range.

In conclusion, the following modelling recommendations should be implemented to capture and subsequently reduce the acoustic boom in a passenger car:

1. Abaqus 'strongly coupled' solution procedure should be implemented to effectively model the vibro-acoustic coupling in the passenger car model.

2. The acoustic mesh for the passenger car should be modeled with a gap between the rear seats and trunk with the speaker openings on the rear package shelf acting as the only connection between trunk and the rest of cavity.

3. Optimal size of speaker openings on the rear package shelf can help in reducing the acoustic response at driver's right ear.

4. Door Cavities also influence the acoustic response at driver's right ear and the acoustic mesh should be modeled accurately. 


\section{6: References}

[1]: "Vehicle Noise Quality and Satisfaction Data", J.D. Power and Associates. McGrawHill. http://www.jdpower.com/corporate/automotive

[2]: Shorter, Phil, "Recent Advances in Automotive Interior Noise Prediction", Noise and Vibration Conference, Brazil, 2008

[3]: Van Karsen, C., DeClerck, J., Odegard, G., Dhabe, S., Pathak, M., "A Study of Passenger Car Acoustic Cavity Boom Simulation Methods", Report submitted to General Motors, Dec 2013

[4]: Nefske, D.J., Wolf Jr, J.A. and Howell, L.J., "Structural-acoustic finite element analysis of the passenger compartment: a review of current practice", Journal of Sound and Vibration, January/February 1982

[5]: Yashiro, H., Suzuki, K., Kajio, Y., Hagiwara, I., and Arai A., "An application of structural-acoustic analysis to car body structure”, SAE paper 850961, 1985

[6]: Lalor, $\mathrm{N}$ and Priebsch, H-H, "The prediction of low and mid-frequency internal road vehicle noise: a literature survey", Proceedings of the Institution of Mechanical Engineers, Part D: Journal of Automobile Engineering, 221, (3), 245-269, 2006

[7]: Abaqus 6.12 Documentation

[8]: Heylen, W., Lammens, S., Paul, S., "Modal Analysis Theory and Testing”, Katholieke Universiteit Leuven, Departement Werktuigkunde, 1999

[9]: Logan, Daryl L., "A First Course in the Finite Element Method, Fifth Edition", Thompson, Toronto, 2012

[10]: LMS Virtual.lab Documentation

[11]: Altair Hypermesh 11.0 Documentation

[12]: Van Karsen, C., “MEEM 5702 Numerical Vibro-Acoustics”, Class Notes, 2012

[13]: Van Karsen, C., DeClerck, J., Dhabe, S., "Testing and Model Correlation of a Plexiplate with a Water Boundary Condition", IMAC XXXII conference, Florida, USA, 2014

[14]: Heylen, W., and Avitable, P., "Correlation considerations - part 5 (degree of freedom correlation techniques)", IMAC - XVI, 1998 
http://sem-proceedings.com/16i/sem.org-IMAC-XVI-16th-Int-160805-Degree-OfFreedom-Correlation-Techniques.pdf

[15]: Allemang, Randall J., University of Cincinnati, Cincinnati, Ohio, "The Modal Assurance Criterion -Twenty Years of Use and Abuse", Sound and Vibration, August 2003

[16]: Sung, S., Nefske, D. J., Le-The, H., and Bonares, F., "Development and experimental evaluation of a vehicle structural-acoustic trimmed body model", SAE paper 991798, 1999

[17]: Stokes, W., Bretl, J., Crewe, A., Park, W. S., Lee, J.Y., and Lee, M. S., "Computer simulation of in-vehicle boom noise", SAE Paper 971914, 1997 